\title{
Novel Method for Mitigating Injectivity Issues during Polymer Flooding at High Salinity Conditions
}

\author{
By \\ Julia Maria Schmidt \\ in partial fulfilment of the requirements for the degree of
}

\section{Master of Science}

in Applied Earth Sciences

at the Delft University of Technology,

to be defended publicly on Thursday July 26,2018 at 15:00 PM.

Supervisor:

Prof. Dr. Pacelli Zitha

Delft University of Technology

Mohsen Mirzaie Yegane

Delft University of Technology

Dr. Marita Neuber

TouGas Oilfield Solutions GmbH

Dr. Fatima Dugonjic-Bilic

TouGas Oilfield Solutions GmbH

Benjamin Gerlach

TouGas Oilfield Solutions GmbH

Thesis committee:

Prof. Dr. Pacelli Zitha (Chair)

Delft University of Technology

Prof. Dr. Bill Rossen

Delft University of Technology

Dr. Anne-Catherine Dieudonné

Delft University of Technology

Dr. Ali Fadili

Shell Global Solutions B.V.

Dr. Marita Neuber

TouGas Oilfield Solutions $\mathrm{GmbH}$

Mohsen Mirzaie Yegane

Delft University of Technology

An electronic version of this thesis is available at http://repository.tudelft.nl/.

\section{T̛UDelft}

\section{TOU GAS}

Oilfield Solutions 


\section{Acknowledgements}

This thesis has been completed as a cooperation between TU Delft and TouGas Oilfield Solution GmbH.

I would first like to express my sincerest gratitude to my advisor, Prof. Dr. Pacelli Zitha, for making it possible to write this thesis as a collaborative research project between TU Delft and TouGas Oilfield Solution GmbH. His guidance helped me throughout my research, manifesting in the completion of this thesis. I would also like to thank my advisor, Mohsen Mirzaie Yegane, from TU Delft not only for his insightful comments and guidance, but also for his challenges, which encouraged me to critically look at my research project from various perspectives.

Further, I would like to thank the Head of Research and Development, Dr. Marita Neuber, for the possibility of completing my thesis at TouGas and for granting me full access to the laboratories and company resources. Her guidance and invaluable experience in applied polymer science helped me a lot in planning and in keeping my progress on schedule. I would also like to thank the head of the laboratory, Dr. Fatima Dugonjic-Bilic and Benjamin Gerlach, for the endless hours they have spent in discussing and setting up measurement procedures and test evaluations with me. Their valuable support throughout the entire thesis helped me to develop, broaden and reach my research goals. I also want to thank my fellow lab-mates at TouGas for assisting me with the equipment handling in the laboratories and other technical issues at all times. 
Table of Content

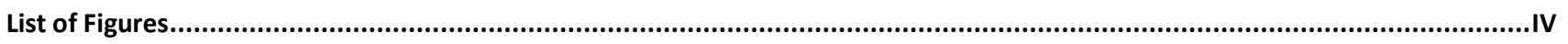

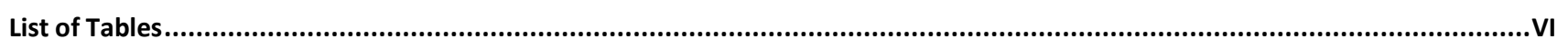

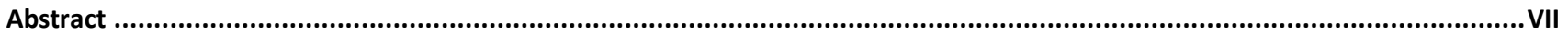

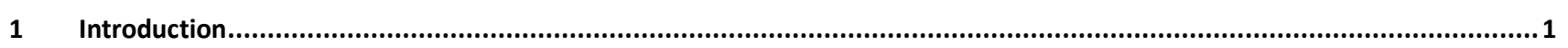

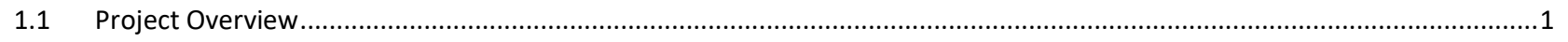

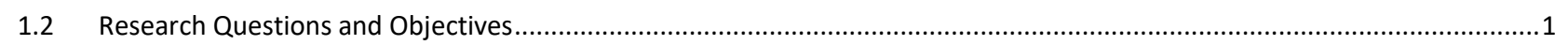

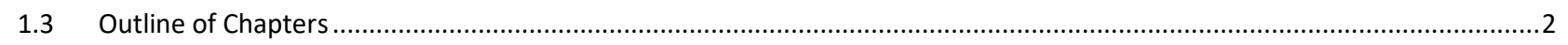

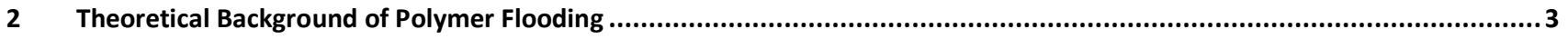

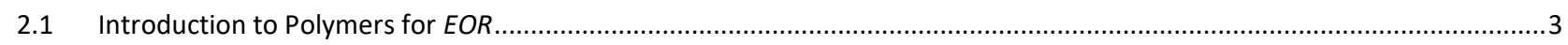

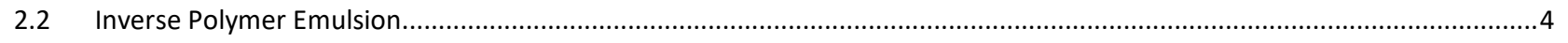

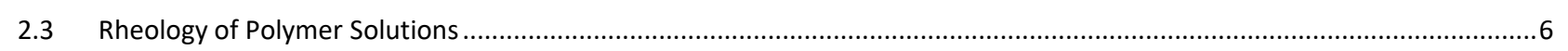

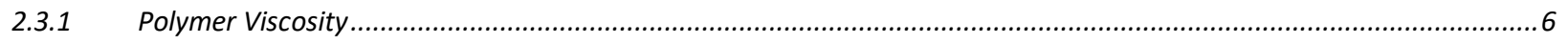

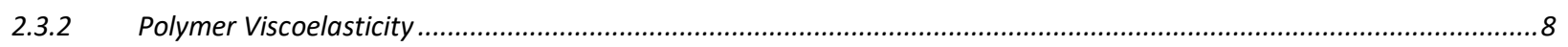

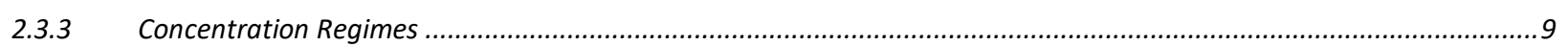

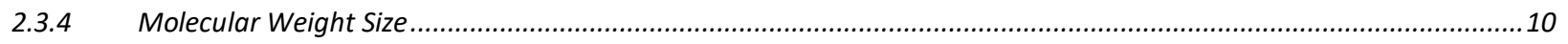

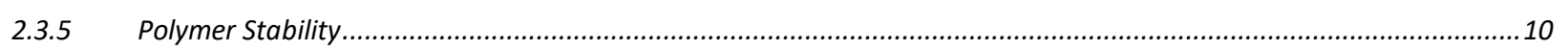

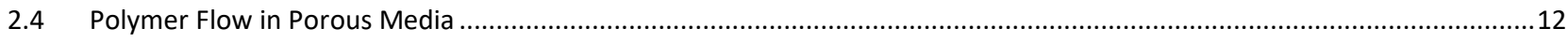

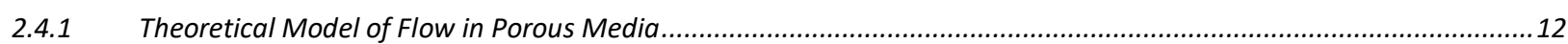

2.4.2 Injectivity Challenges of High-Molecular-Weight Polymer Solutions in Porous Media............................................... 14

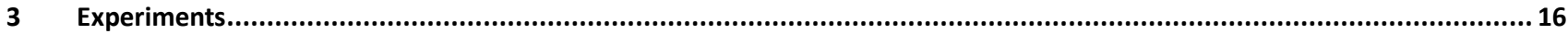

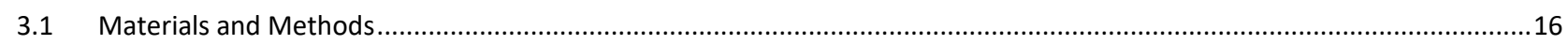

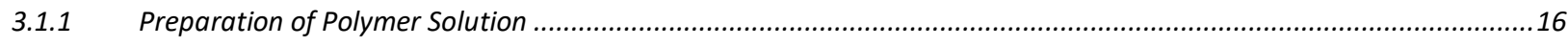

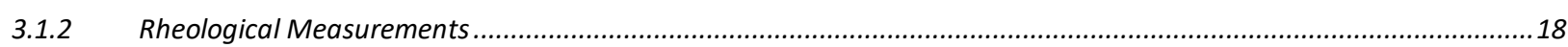

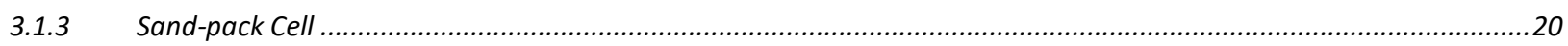

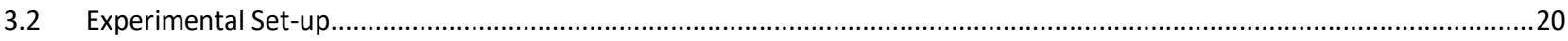

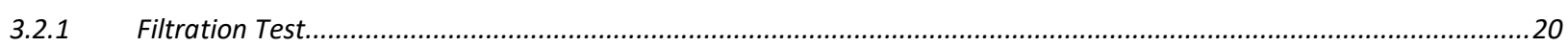

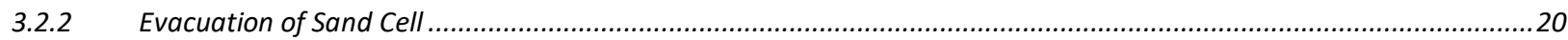

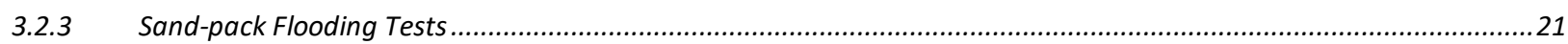

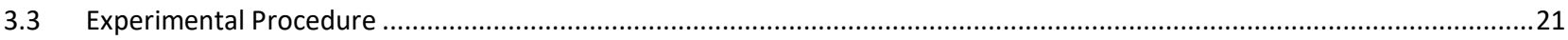

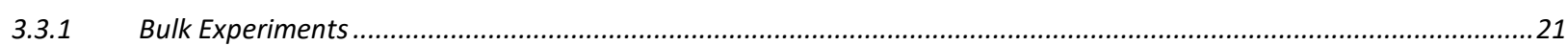

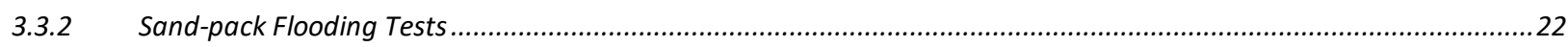

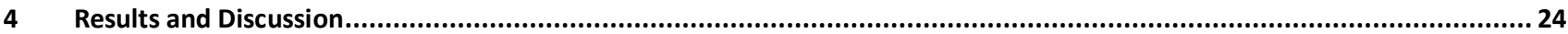

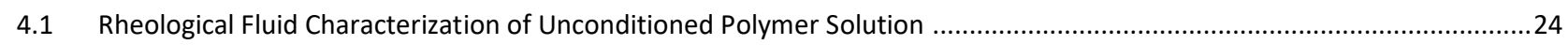

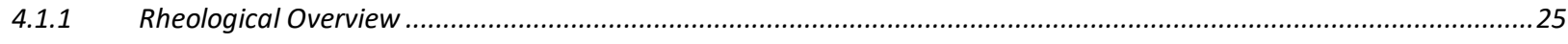

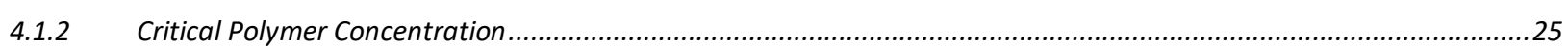

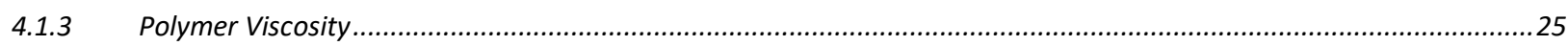

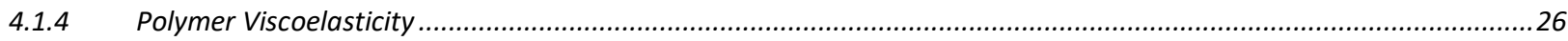

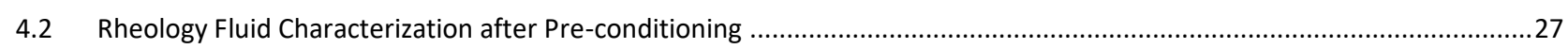

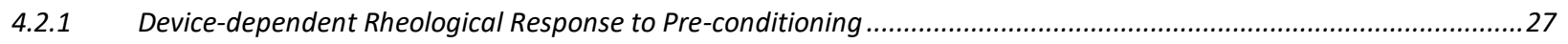

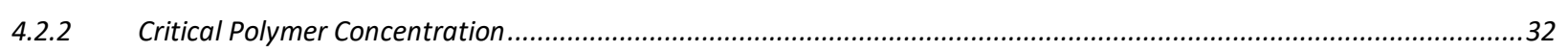

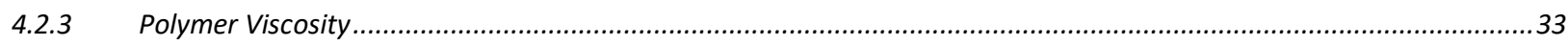

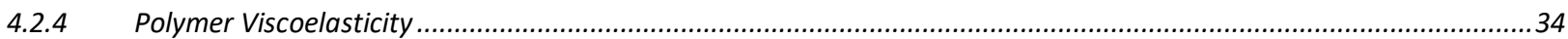




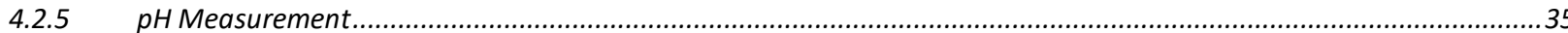

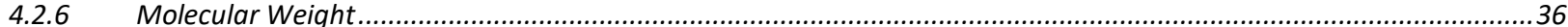

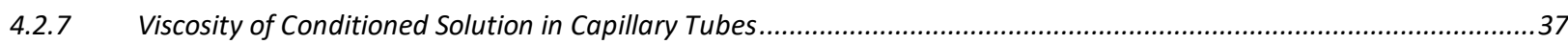

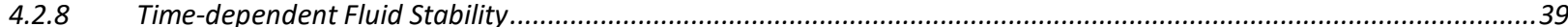

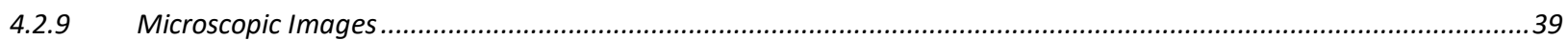

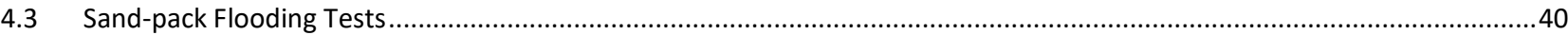

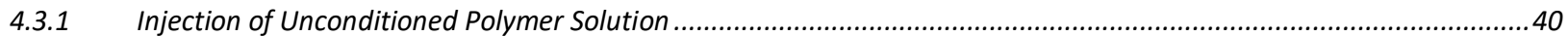

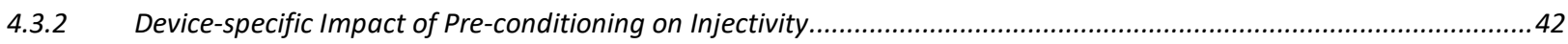

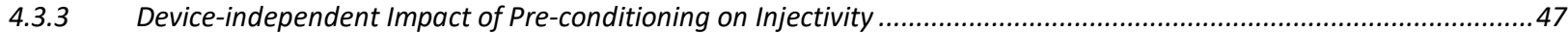

4.3.4 Impact of Polymer and Surfactant Concentration on Pre-conditioned Polymer Solution ............................................53

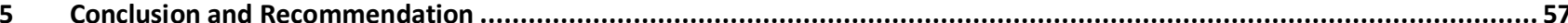

Nomenclature.........................................................................................................................................

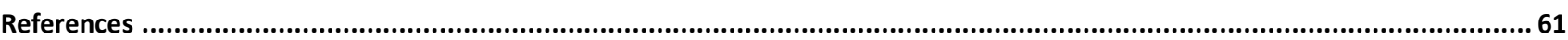




\section{List of Figures}

Figure 2-1: Chemical structure of xanthan (left) and HPAM (right) (Sheng, 2010) .......................................................................

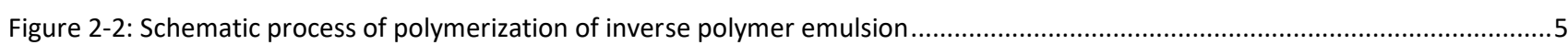

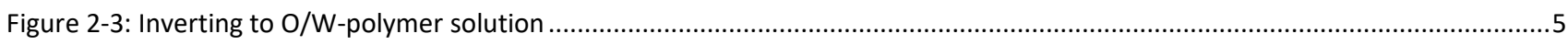

Figure 2-4: Viscosity development of synthetic polymer during flow through porous media, adapted from Chauveteau, 1986 and Sheng,

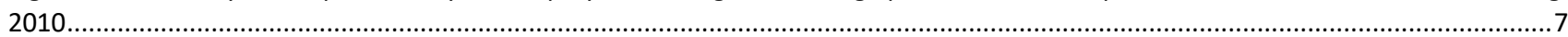

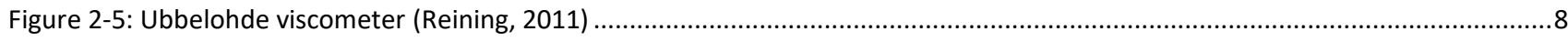

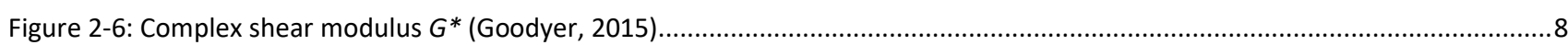

Figure 2-7: Determination of gel-like and liquid-like structure by measuring storage modulus $G^{\prime}$ and loss modulus $G^{\prime \prime}$ against shear

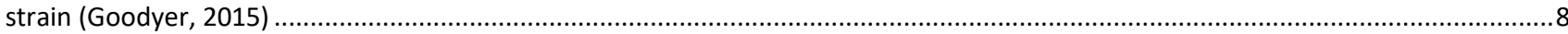

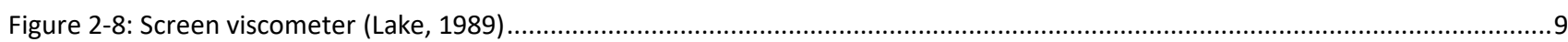

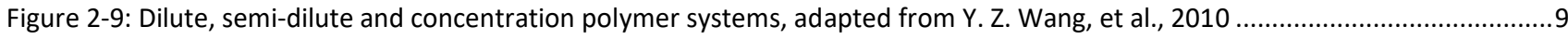

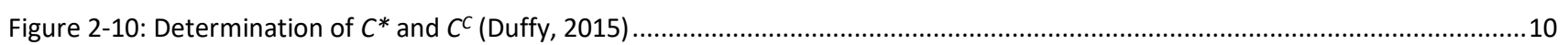

Figure 2-11: Molecular-weight distribution for HPAM-based polymer solutions adapted from Sorbie (1991) ....................................10

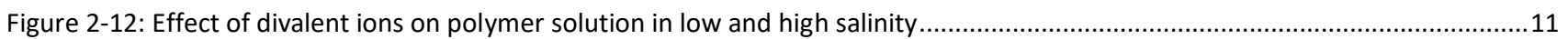

Figure 2-13: Chemical structure of acrylate, $n V P$ and ATBS, adapted from (Zaitoun, et al., 2011) ....................................................11

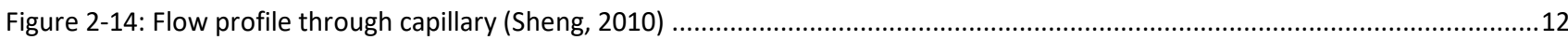

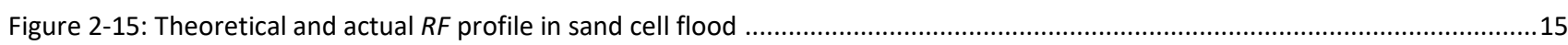

Figure 2-16: Schematic of reasons for pre-conditioning the polymer solution: polymer stretching with increasing shearing [a], narrowing

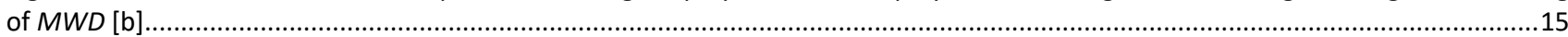

Figure 3-1: Preparation of unsheared polymer solution with magnetic stirrer and conditioned polymer solutions with three shearing

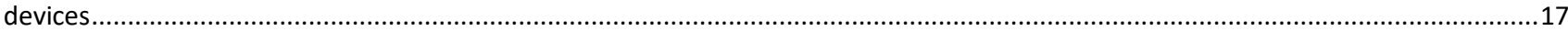

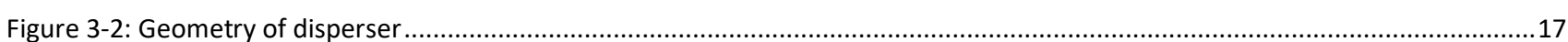

Figure 3-3: Ultra-Turrax S $20 \mathrm{~N}$ - $18 \mathrm{G}$ assembled in dispersion tool [a], turrax [b], schematic representation of shearing between stator

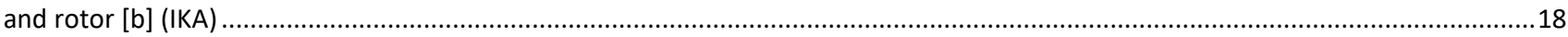

Figure 3-4: Schematic representation of experimental set-up of capillary tubes [a], experimental set-up of capillary tubes [b] ...........18

Figure 3-5: Polymer separation in ethanol [a], washing separated polymer with suction filter [b], dried polymer [c], dissolved in distilled

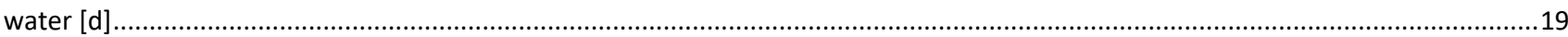

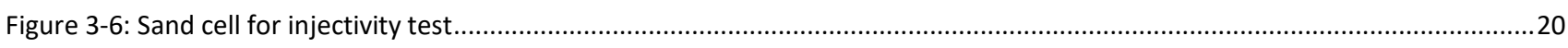

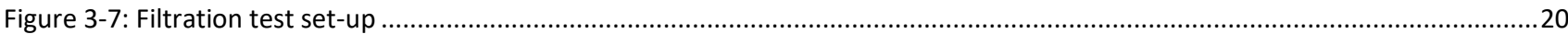

Figure 3-8: Vacuum pump attached to sand cell [a] and experimental set-up of sand-pack flooding test [b]...................................21

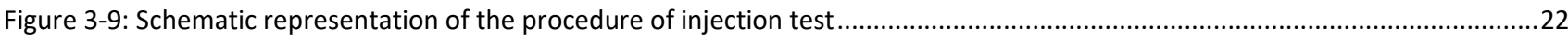

Figure 3-10: Schematic set-up of sand-pack flooding test at different stages of injection, referring to Table 3-4 ..............................23

Figure 4-1: Viscosity measured by Ubbelohde vs polymer concentration - no pre-shearing; only inversion with magnetic stirrer with

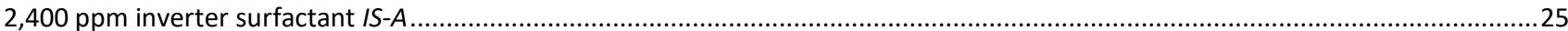

Figure 4-2: Viscosity stability at changing shear rate of unsheared polymer solution, running the solution from low to high, high to low, low to high, high to low shear rate measured with the Anton Paar rheometer, and viscosity measured with the Ubbelohde viscometer

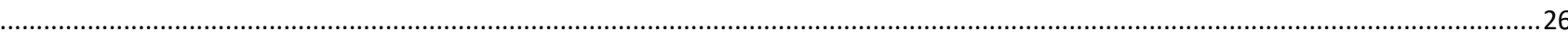

Figure 4-3: Viscoelasticity measurements of differently sheared polymer solutions at constant frequency . .26

Figure 4-4: Viscosity measured by Ubbelohde and resulting mechanical degradation from the Ubbelohde [a] and screen factor and screen factor loss [b] from experiment series with disperser...

Figure 4-5: Viscosity measured by Ubbelohde and resulting mechanical degradation from Ubbelohde [a] and screen factor and screen factor loss [b] from experiment series with turrax.

Figure 4-6: Viscosity measured by Ubbelohde and resulting mechanical degradation from Ubbelohde [a] and screen factor and screen factor loss [b] from experiment series with capillary

Figure 4-7: Rheological master curve between viscosity measured by Ubbelohde and screen factor after shearing polymer solution with different shearing devices. 
Figure 4-8: Turbine-power correlation for disperser and turrax adapted from Hershey \& Brodkey (1988), Bartes, Fondy \& Corpstein

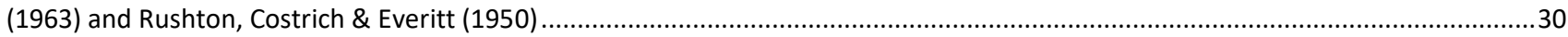

Figure 4-9: Master curve of mechanical degradation against Ev [a], and viscosity measured with Ubbelohde and screen factor against Ev [b] for disperser and turrax......

Figure 4-10: Master curve of mechanical degradation against Ev [a], and viscosity measured with Ubbelohde and screen factor against Ev [b] for disperser, turrax and capillaries

Figure 4-11: Viscosity from Ubbelohde vs polymer concentration - differently pre-sheared polymer solutions with 2,400 ppm inverter surfactant $I S-A$.

Figure 4-12: Viscosity at $250 \mathrm{rps}$ from R/S+ Rheometer vs polymer concentration measured with Brookfield R/S+ Rheometerdifferently sheared polymer solutions with 2,400 ppm inverter surfactant $I S-A$.

Figure 4-13: Viscosity curves with increasing shearing intensity measured with Anton Paar M 302.

Figure 4-14: Viscoelasticity measurements of differently sheared polymer solutions at constant frequency measured with Anton Paar M 302

Figure 4-15: pH after pre-shearing with different shearing intensities and devices 36

Figure 4-16: Molecular weight measured for differently sheared polymer solutions versus accumulated energy input during preshearing

Figure 4-17: Relative apparent viscosity vs shear rate [a], relative apparent viscosity vs pressure drop [b] .....................................38

Figure 4-18: Viscosity measured with Ubbelohde and molecular weight vs pressure drop for capillary tube with $1 \mathrm{~mm}$ diameter and 10 $\mathrm{cm}$ length.

Figure 4-19: Pre-sheared polymer solution at day 0 [a] and day 13 [b] and Polymer stability of sheared polymer solution over time

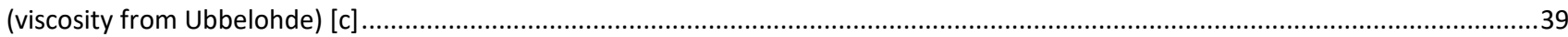

Figure 4-20: Oil droplets in unsheared [a] and strongly sheared [b] polymer solution ................................................................39

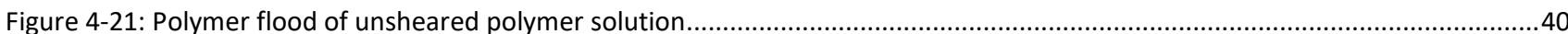

Figure 4-22: Unsheared polymer solution separated by density difference over 12 days ............................................................41

Figure 4-23: Polymer flooding test of unsheared polymer solution at different surfactant concentrations.......................................42

Figure 4-24: Injectivity results of unsheared and disperser-sheared polymer solutions .................................................................43

Figure 4-25: Injectivity results of unsheared and turrax-sheared polymer solutions ..................................................................44

Figure 4-26: Injectivity results of unsheared and capillary-sheared polymer solutions .....................................................................45

Figure 4-27: Injectivity results of unsheared and disperser-turrax-sheared polymer solutions ........................................................46

Figure 4-28: Impact of pre-shearing on viscosity, screen factor and injectivity ...........................................................................

Figure 4-29: Injectivity results of unsheared and sheared polymer solutions of different shearing origins and intensities...................48

Figure 4-30: Viscosity from Ubbelohde and injectivity gradient after $30 \mathrm{PV}$ of polymer-injected versus screen factor of carried out

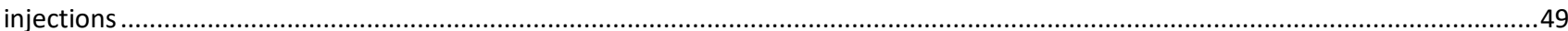

Figure 4-31: Injectivity results of polymer solutions of the same unsheared polymer solution, but different $\mathrm{pH}$ adjustment $(\mathrm{M}$ has a pH of 7.4 without $\mathrm{pH}$ adjustment, $\mathrm{M}-1$ has a pH of 8.1 with pH adjustment) [a], and injectivity results of polymer solutions of the same polymer solution with the same shearing intensity and device, but different pH adjustment ( $\mathrm{T} 2$ has a pH of 7.3 without pH adjustment, T2-1 has a $\mathrm{pH}$ of 8.0 with $\mathrm{pH}$ adjustment) [b]... . .50

Figure 4-32: Viscosity measured by Ubbelohde and screen factor vs mechanical degradation in the sand-pack at $0.5 \mathrm{ml} / \mathrm{min}$ using injected solutions M, C, T1, T2, D, DT1, DT2, DT3, DT4, T3 shown in Figure 4-29 and Table 4-8

.51

Figure 4-33: \%-permeability reduction determined by the inverse of the ratio of theoretical and measured $R F$ after injecting the sheared solutions C, T1, T2, D, DT1, DT2, DT3, DT4, T3 (shown in Figure 4-29 and Table 4-8) at 3 and 30 PV as well as the residual reduction .52

Figure 4-34: Disperser-turrax-sheared polymer injection (sheared $30 \mathrm{~min}$ at $1750 \mathrm{rpm}$ with disperser and $1 \mathrm{~min}$ at 20,000 rpm turrax)

Figure 4-35: Injectivity results of polymer solutions with different polymer concentration with the same shearing intensity and devices

Figure 4-36: Non-inverted polymer solutions: fresh polymer solution just before injection [a], polymer solution after a few hours with precipitated polymer and emulsifiers [b]

Figure 4-37: Injectivity results of polymer solutions sheared the same way, with the same polymer concentration of 4,000 ppm and different surfactant concentration [a], and Injectivity results of polymer solutions, sheared the same way with different surfactant concentration. $\mathrm{D}$ is the fresh polymer solution $P S-A$, and D-1 is $P S-A$ separated over 13 days with a separator funnel [b] ..................56 


\section{List of Tables}

Table 3-1: Components of polymer solution and used amount for polymer solution PS-A …......................................................16

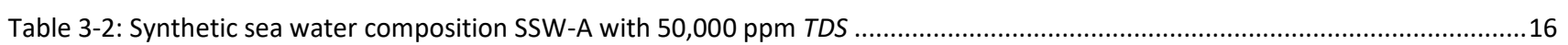

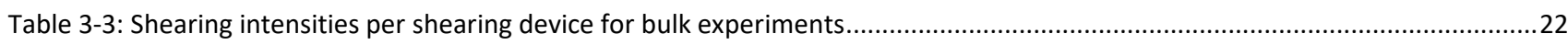

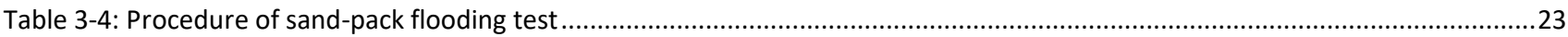

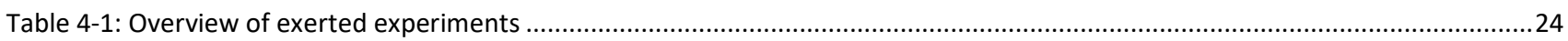

Table 4-2: Rheological parameters of unsheared separated polymer solution ..................................................................................

Table 4-3: Rheological parameters of unsheared polymer solutions (I. with 2,400 ppm IS-A, II. with 2,400 ppm IS-A and then separated

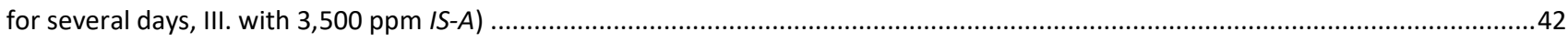

Table 4-4: Rheological results of injected polymer solutions - unsheared and disperser-sheared, referring to Figure 4-24.................43

Table 4-5: Rheological results of injected polymer solutions - unsheared and turrax-sheared, referring to Figure 4-25......................44

Table 4-6: Rheological results of injected polymer solutions - unsheared and turrax-sheared, referring to Figure 4-26......................45

Table 4-7: Rheological results of injected polymer solutions - unsheared, disperser-sheared and disperser-turrax-sheared, referring to

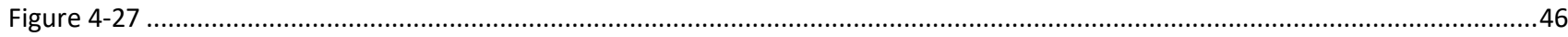

Table 4-8: Rheological results of injected polymer solutions - unsheared and differently sheared, referring to Figure $4-29 \ldots \ldots \ldots \ldots \ldots . . .48$

Table 4-9: Rheological results of injected unsheared polymer solutions with different inverter surfactant concentration, referring to

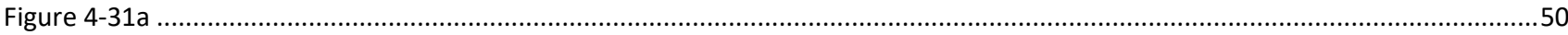

Table 4-10: Rheological results of injected polymer solutions with the same shearing intensity but different inverter surfactant

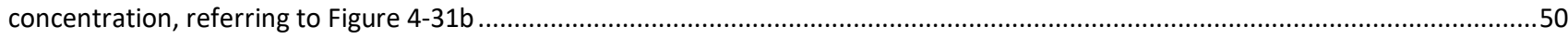

Table 4-11: Nitrogen analysis of flooding test with a sheared polymer solution in a 1,000 mD permeable sand-pack .......................52

Table 4-12: Rheological results of injected polymer solutions with different polymer concentration with the same shearing intensity and devices, referring to Figure 4-35.

Table 4-13: Rheological results of injected polymer solutions with the same shearing intensity but different inverter surfactant concentration, referring to Figure 4-37aError! Reference source not found.

Table 4-14: Rheological results of injected polymer solutions with the same shearing intensity but different inverter surfactant concentration, referring to Figure 4-37b. 


\section{Abstract}

Synthetic polymers, in the emulsified form, have been utilized for enhanced oil recovery applications by using saline make-up water. However, there are concerns that have been raised about their injectivity. The large entangled polymer chains can clog the pore throats, giving a tendency to cause injectivity reduction. In this study, processing techniques were used to condition an acrylamide-based copolymer inverse emulsion system at a salinity of 50,000 ppm TDS before being injected into porous media.

The investigated polymer solution contained 4,000 ppm active emulsion-polymer and 2,400 ppm surfactant, providing a zero-shear rate viscosity of $13 \mathrm{mPas}$. Shearing with two agitators, a disperser and Ultra-Turrax, at different intensities and pressure-driven flow into a thin capillary reduces the size of the largest polymer and disentangles the polymer chains while maintaining its viscosifying power as much as possible. Subsequently, the filtration ratios $(F R)$ with optimum between 1-1.2 were determined by performing filtration tests in a 1-micron polycarbonate membrane to evaluate the plugging behavior. This was followed by sand-pack flooding tests of differently sheared solutions in order to investigate the impact of pre-conditioning on injectivity.

Bulk experiments enabled the establishment of master curves showing viscosity and screen factor dependences on accumulated energy during pre-shearing, regardless of shear origin. The injected unsheared polymer solution has an $F R$ of 1.6 and an injectivity gradient, e.g. ratio of resistance factor over 10 pore volumes, of 2.4. All injected preconditioned solutions have an $F R$ in the optimal range between 1 to 1.2 . By imposing $15 \mathrm{MJ} / \mathrm{m}^{3}$, the disperser-sheared solution improves the injectivity by decreasing the injectivity gradient to 1.3 , while the viscosifying power is reduced by $2 \%$ and the screen factor by $30 \%$. To reach the same injectivity gradient of 1.3 with Ultra-Turrax, $31 \mathrm{MJ} / \mathrm{m}^{3}$ were imposed, which reduces the viscosity and screen factor by $11 \%$ and $44 \%$ respectively. The sheared solution into a capillary imposes $50 \mathrm{MJ} / \mathrm{m}^{3}$, giving an injectivity gradient of 2.7 . Both viscosity and screen factor are reduced by $19 \%$ and $53 \%$ respectively. This indicates that the injectivity performance is shear-origin dependent and the resulting polymer structure, when sheared through contractions, has a different alignment as compared to shearing with the agitators, the disperser and Ultra-Turrax.

In conclusion, the rheological dependencies of sheared polymer solutions form a master curve dependent of accumulated energy during shearing with different shearing devices. Further, the proven beneficial impact of pre-conditioning with agitators before injection enables a better utilization of polymer flooding operations by reducing the risk of pore plugging. 


\section{Introduction}

Enhanced oil recovery $(E O R)$ is the employment of various techniques in order to increase the amount of crude oil that can be produced from a reservoir. It is usually performed at a later stage of the field's life time, and it can be divided into three types: thermal EOR, gaseous EOR and chemical EOR. Polymer flooding is the most widely used chemical EOR technique, which aims at increasing the recoverable hydrocarbons by enhancing the viscosity of the drive fluid.

Challenges are presented during the application of polymer flooding under harsh environmental conditions, which cannot provide stable conditions, adequate space for the polymer fluid preparation, and fresh water. In practice, mostly powder polymers are used. Yet, emulsion polymers can be preferably used at unstable conditions, where there is a lack of space for polymer preparation.

When polymer flooding operations are performed at difficult conditions, the polymers need to withstand for instance high saline make-up water that is present at offshore locations in sea water. The water-soluble polymer chains are highly coiled and entangled due to hydrogen-bonding and interactions of the polymers with divalent ions present in saline environments, causing pore plugging due to the large hydrodynamic radius. Further, the high-end of the molecular weight distribution (MWD) additionally leads to pore plugging in porous media.

By pre-conditioning the polymer solution before its injection into porous media, the polymer chains can be disentangled and elongated. Further, rupturing of the chains occurs preferentially at the high-end of the MWD, meaning that the distribution is narrowed by shear stress, as Maerker (1975), among other researchers, had discovered in early studies. Thus, the deliberate pre-conditioning of the polymer solution before the polymer flooding operation can have a beneficial impact on the injectivity.

\subsection{Project Overview}

The system that is being investigated is a high-molecular-weight acrylamide-based copolymer inverse emulsion system. The polymer system contains two phases that are stabilized by surfactants. Water forms the continuous phase and contains water-soluble polymers, while oil droplets are dispersed in the water phase. Throughout the study, the concentration of the polymer and of the surfactant are kept constant at 4,000 ppm and 2,400 ppm respectively. The make-up water that is employed is synthetic sea water having 50,000 ppm total dissolved solids (TDS).

In this study, pre-conditioning is performed by shearing the polymer solution with three different shearing devices at various intensities. The shearing devices that are employed are a disperser and Ultra-Turrax, which are two different agitator systems, and a pressure-driven flow into a capillary tube.

\subsection{Research Questions and Objectives}

This thesis aims at investigating the injectivity behavior of polymer solutions by conditioning the solutions before injection. The main objectives of the thesis are as follows:

(1) Prove that pre-conditioning improves the injectivity performance of the polymer solution without significantly destroying its viscosifying effect

(2) Find dependencies and patterns between rheological parameters and the injectivity of differently pre-sheared polymer solutions 
The thesis is divided into two parts: bulk and sand-pack flooding experiments. In the bulk experiments a detailed rheological analysis is carried out, capturing the response of pre-conditioning the solution with the three shearing devices. This gives an understanding of its rheological response. Thereby, viscosity, polymer stability and the imposed energy input by pre-shearing are determined, which allows for studying the dependencies and correlations between the rheological parameters. On the basis of the bulk experiments, flooding tests in 1,000 mD sand-packed cells are performed. The injectivity behavior for differently pre-conditioned polymer solutions are studied by observing dependencies between the injections and by drawing conclusions to the bulk experiments.

\subsection{Outline of Chapters}

The thesis consists of a theoretical introduction in Chapter 2, covering a brief overview of polymer flooding techniques and the challenges faced in doing so. In Chapter 3, the experimental set-up, including bulk and sand-pack flooding experiments, is described. In Chapters 4 , the results of the bulk and sand-pack flooding experiments are presented. The final chapter presents the conclusions for this research and recommendations for future research. 


\section{Theoretical Background of Polymer Flooding}

This thesis focuses on polymer flooding, the most commonly used chemical EOR technique. Through this technique, the mobility of aqueous drive fluid is reduced by adding viscosity-enhancing polymers. This improves the displacement stability during the operation by creating a sharper flooding front, reducing the bypassing of oil, and increasing the sweep efficiency.

This chapter provides a brief overview of the polymers used in the EOR industry and the studied inverse polymer emulsion system. The rheology of polymers is introduced, including the viscosity and viscoelasticity behavior, as well as the polymer stability. Further, the flow through porous media is discussed, covering the basics of the theoretical polymer flow through porous media, and the related challenges faced regarding polymer injectivity in porous media are defined.

\subsection{Introduction to Polymers for EOR}

Polymers for EOR can be classified as biopolymers and synthetic polymers. Biopolymers, such as xanthan, have a rigid structure, which aligns in flow direction. Thus, they are not affected significantly by mechanical degradation, but they are prone to biological or chemical degradation. As opposed to biopolymers, synthetic polymers, such as hydrolyzed polyacrylamide (HPAM), are sensitive to mechanical degradation, since they do not align perfectly in accordance with the flow direction due to their flexible coiled chain structure, and it is thereby affected by extensional shear flow. Thus, the synthetic polymer coils are completely stretched, followed by a rupturing of the chain at the center and permanent loss of viscosity (Jouenne, Chakibi, \& Levitt, 2017; Sheng, 2010). The chemical structure of xanthan and HPAM are shown in Figure 2-1.

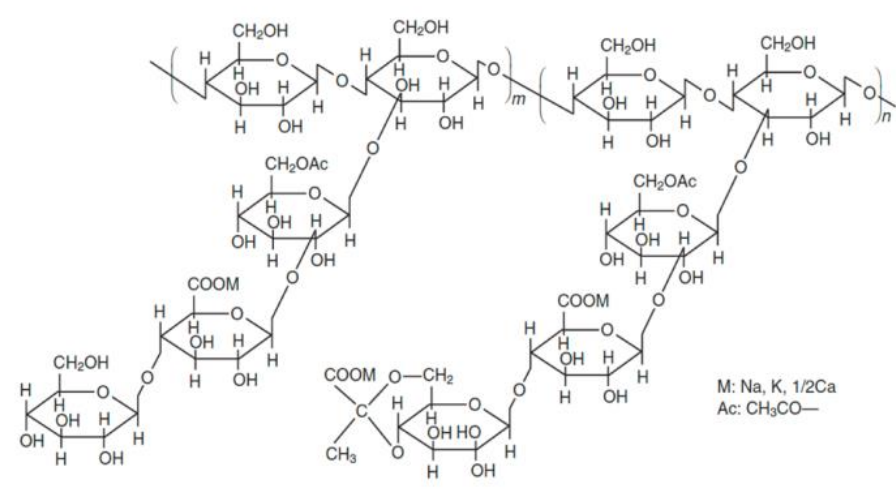

Xanthan

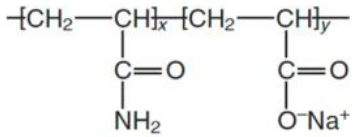

Partially hydrolyzed

Polyacrylamide (HPAM)

Figure 2-1: Chemical structure of xanthan (left) and HPAM (right) (Sheng, 2010)

Due to the susceptibility of xanthan to bacterial attacks, HPAM is mainly used for EOR. The hydrolysis converts in commercial products $15-35 \%$ of the amide groups, $\mathrm{CONH}_{2}$, to anionic carboxyl groups, COO-. Thus, HPAM molecules and other synthetic polymers are negatively charged. The presence of the -O- of the carboxyl group in the HPAM allows it to withstand higher temperatures as compared to xanthan. Further, $\mathrm{CONH}_{2}$ reduces the precipitation of cations, such as $\mathrm{Ca}^{2+}$ and $\mathrm{Mg}^{+}$, providing a higher salinity stability. However, it reduces the viscosifying power at the same time, since there is only a little repulsion between the chains due to the change of charge. Polymer chains, such as HPAM, dissolved in water are not perfectly stretched and elongated due to the effect of hydrogen bridging, which causes the formation of dipole-dipole interactions between $\mathrm{H}_{2} \mathrm{O}$ molecules and the negatively-charged groups. Depending on the type of monomers and their charge, intramolecular (i.e. only in the polymer chain itself) and intermolecular (i.e. with other 
polymer chains) interactions occur, which are strong enough to coil the polymer (Chauveteau, 1986; Sheng, 2010; Sorbie, 1991).

The most common physical form of polymers is powder form. They are especially used onshore as they can be easily transported and stored at a low cost. However, powder polymers require a more elaborate and sophisticated preparation in large mixing units at the given location as they need long stirring time at stable conditions. The alternative to powder polymers are emulsion polymers, which contain polymers in the water phase, stabilized by the addition of surfactants in the continuous oil phase. The application of powder or emulsion polymers strongly depends on the location of the production site. In general, emulsion polymers have a higher OPEX as they are more expensive and a larger amount of volume has to be transported. But, the advantage of an emulsion is that the preparation of the polymer solution at the production site is comparably easy-to-handle. At onshore locations powder polymers are usually used, since there is enough space to set up preparation facilities, the conditions are stable, and the transportation costs are comparably cheaper. For offshore operations, however, it can be more feasible to use emulsion polymers, both in terms of economic and technical factors, due to the convenience in the preparation and the reduced need of space for adequate mixing facilities (Jouenne, Klimenko, \& Levitt, 2016; Lake, 1989).

\subsection{Inverse Polymer Emulsion}

The polyacrylamide-based polymer is stable at high salinities up to 200,000 ppm, which allows for the use of brine water as make-up water for the polymer solution. The inverse polymer emulsion is a water-in-oil emulsion. An organic oil phase forms the continuous phase, while water-soluble polymers based on acrylamide are dissolved in the water phase. The addition of emulsifiers enables the stabilization of water droplets throughout the oil phase. A combination of water-and oil-soluble emulsifiers are used, whereby its solubility is determined by the hydrophilic-lipophilic balance $(H L B)$. An $H L B$ of 0 means that an emulsifier is totally oil soluble, while an $H L B$ of 20 suggests that the emulsifier is totally water soluble. The process of polymerization is shown schematically in Figure 2-2. The water-soluble monomers are dissolved in water, while the two emulsifiers are dissolved in oil. A pre-emulsion is created through continuous stirring, whereby the emulsifiers form micelles around the water droplets that contain the monomers. The lipophilic tail of the emulsifiers lies at the outside of the water droplet in the oil phase, while the hydrophilic heads of both emulsifiers protrude into the water phase. The combination of the two emulsifiers was chosen since it allows to pack more emulsifiers closely together due to the difference in size between the hydrophilic head and the lipophilic tail. This results in a low interfacial tension, which provides long-term stability for the emulsion. After creating the pre-emulsion, initiators are added. After continuous stirring at high temperature and high $\mathrm{pH}$, the initiators become reactive and react with the remaining monomers in the oil phase. The initiators break the unsaturated double bond in the monomer, which creates a chain reaction as the monomer then has another free electron. This free electron attacks the double bond of another monomer. And, this leads to the formation of water-soluble oligomers, which are forced into the water micelles and subsequently form polymers. The inverse polymer emulsion contains $30 \%$ active polymers. The water droplets in the resulting inverse polymer emulsion are monodispersed in the continuous oil phase and have a diameter of $0.5-1 \mu \mathrm{m}$. The inverse polymer emulsion is then inverted in water through the use of inverter surfactants, creating an oil-in-water solution. 


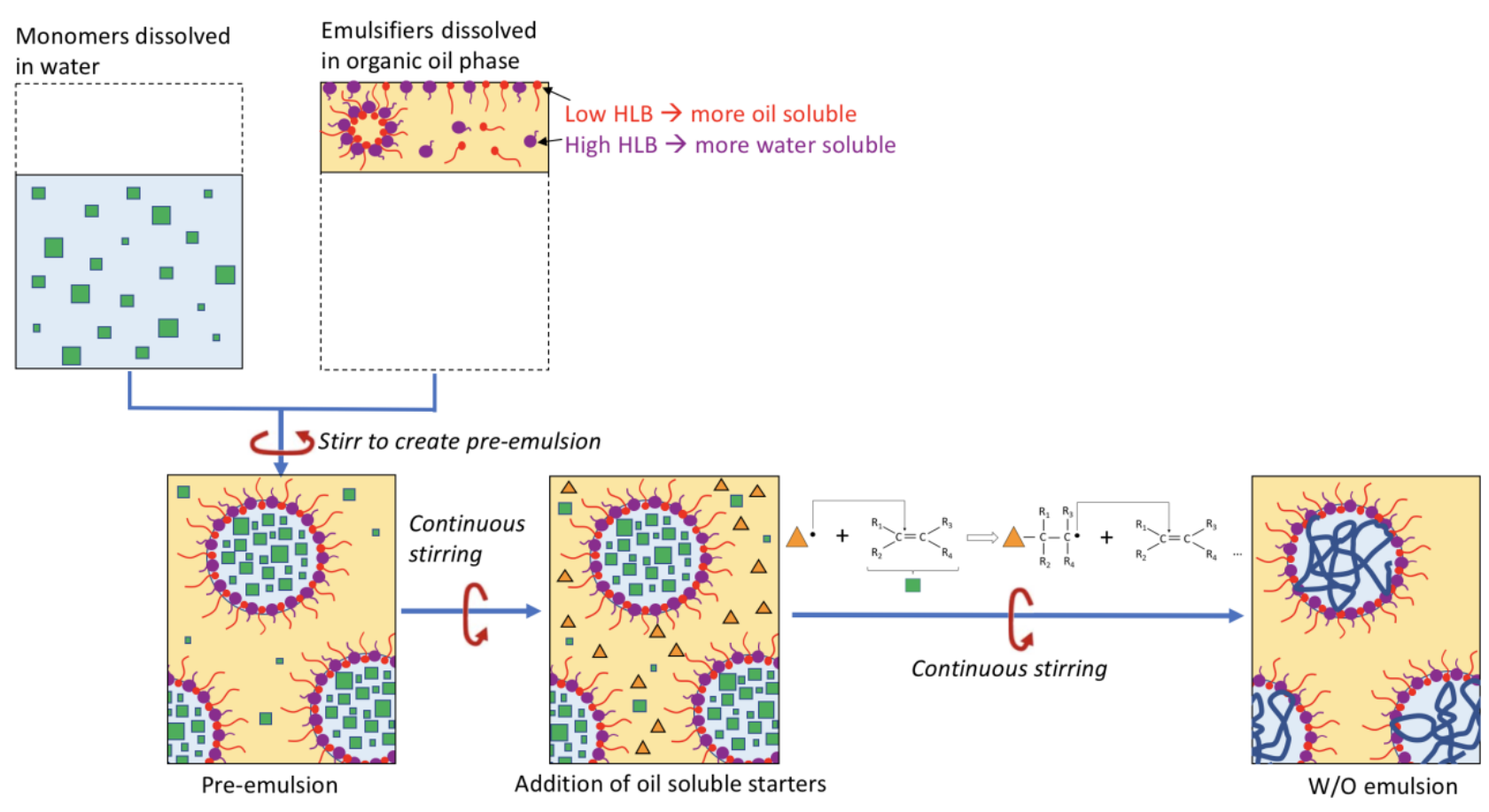

Figure 2-2: Schematic process of polymerization of inverse polymer emulsion

Upon continuous stirring Inverter Surfactant A, IS-A, and active Emulsion Polymer A, EP-A, are added to the Synthetic Sea Water A, SSW-A, (see Figure 2-3). Further stirring of the water-soluble inverters break the emulsion. The oil-soluble emulsifier form micelles around the oil droplets, creating an oil-in-water emulsion. The polymer chains, initially caught in the aqueous micelles, can then expand in the water phase, while still - as explained above - being in a coiled state due to effects of hydrogen bonding effects and salt ions. After being stirred for an adequate time, the polymer solution is fully inverted. The resulting polymer solution is referred to as Polymer Solution A, PS-A.

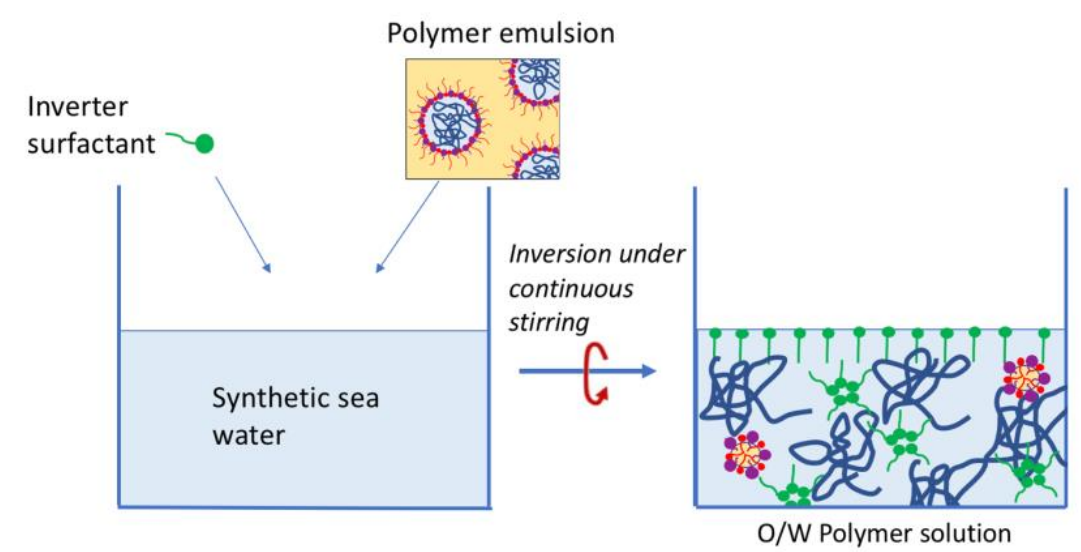

Figure 2-3: Inverting to $\mathrm{O} / \mathrm{W}$-polymer solution 


\subsection{Rheology of Polymer Solutions}

In this section, the basic principles of polymer rheology are introduced. This section encompasses viscosity and viscoelasticity behavior, concentration regimes, molecular weight, and polymer stability.

\subsubsection{Polymer Viscosity}

One of the factors that affects the viscosity of the polymer solution is the shear rate. The shear rate, which the polymer experiences throughout the surface facilities, during injection and in flow through porous media, is subject to change. At low shear rates, Newtonian behavior occurs, while at higher shear rates Non-Newtonian behavior takes place. NonNewtonian behavior can be divided into two types: pseudoplastic and dilatant behavior. Biopolymers only experience pseudoplastic behavior, as the rigid coil structure of the polymer does not allow extensional flow and thus causes a dilatant behavior. Synthetic polymers, such as HPAM, show both pseudoplastic and dilatant behavior (Sheng, 2010; Sorbie, 1991).

The three viscosity regimes of a synthetic polymer solution are shown in Figure 2-4. In the Newtonian regime, the viscosity has a stable value, and it is independent of the shear rate. This behavior occurs at a low velocity and low shear rate, where the motion of the polymer chains is small. The viscosity is proportional to the linear relation of shear stress and the shear rate as shown below:

$$
\tau=\mu \cdot \dot{\gamma}
$$

At a certain first critical shear rate, $\dot{\gamma}_{c 1}$, the fluid starts behaving like a non-Newtonian fluid, and the shear flow dominates over elongational flow. This regime is referred to as shear thinning or pseudoplastic behavior. The viscosity reduces with the increase in shear rate, which occurs for instance during polymer injection. This behavior takes place due to the disentanglement of polymer chains, aligning in the direction of flow, causing a viscosity drop. During injection in the porous media, shear thinning improves the injectivity of polymer solutions as long as no permanent damage is caused to the polymer (Sheng, 2010; Sorbie, 1991).

Shear thickening is exhibited at high shear rates in porous media, and it is owed to the flexible structure of synthetic polymers. This behavior occurs when the relaxation time of polymers is too low, meaning that the polymers do not have enough time to assume its structure when flowing at high shear rates through narrow pore throats, which causes an increase in viscosity (Chauveteau, 1986; Sheng, 2010; Sorbie, 1991).

In this context, there are the following two types of viscosities: shear (or bulk) viscosity, which is observed in a laboratory rheometer or viscometer, and the apparent (or in-situ) viscosity, which is observed during the flow of the polymer solution through porous media. Both viscosities are the same during Newtonian and pseudoplastic behavior. At second critical shear rate, $\dot{\gamma}_{c 2}$, the apparent viscosity shows shear thickening behavior, while the shear viscosity continues to reduce. With regard to polymer flooding in porous media, the apparent viscosity in terms of shear rate is the relevant viscosity, which displays the behavior of the polymer during elongational flow in the reservoir (Chauveteau, 1986; Sheng, 2010; Sorbie, 1991). 
The shear stress with respect to shear rate for Non-Newtonian behavior is provided by the power-law model or the law of Ostwald and de Waele:

$$
\tau=K \cdot \dot{\gamma}^{n}=-K\left(\frac{\partial v_{i}}{\partial r}\right)^{n}
$$

where, $K$ is the consistency factor, and $n$ is the flow factor, which can be fitted to experimental, allowing for the prediction of the viscosity at different shear rates (Chauveteau, 1986; Sheng, 2010). For Newtonian fluids, $K=\mu$ and $n=1$. In the shear thinning regime, the flow factor is $n \leq 1$, and in the shear thickening regime $n>1$ (Sheng, 2010).

Derived from Equation 2-2, the viscosity with respect to shear rate can be expressed as follows:

$$
\mu\left(\gamma^{\cdot}\right)=K \cdot \dot{\gamma}^{n-1}
$$

There are many other models that describe viscosity during shear thinning with respect to shearing, yet the power law model provides the simplest approach, requiring only two unknowns $K$ and $n$. Another frequently used model is the Carreau model given by (Sorbie, 1991):

$$
\mu(\dot{\gamma})=\mu_{\infty}+\left(\mu_{0}-\mu_{\infty}\right)\left[1+(\lambda \dot{\gamma})^{2}\right]^{\frac{n-1}{2}}
$$

requiring four unknowns, where $\eta_{\infty}$ is the infinite shear rate viscosity, $\eta_{0}$ the zero-shear rate viscosity, $\lambda$ a time constant and $n$ the same flow factor, which is included in the power law model. The Carreau model manages to match the viscosity curve also a low and high shear rates and thus provides a better model than the power law model, which only works well for intermediate shear rates (Sorbie, 1991).

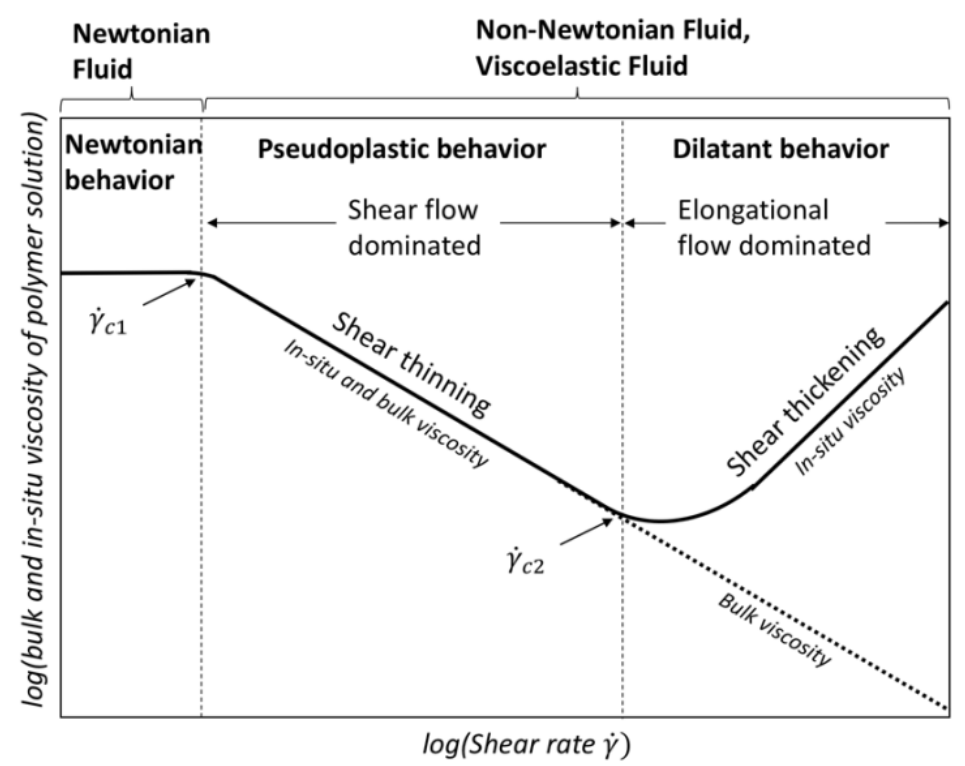

Figure 2-4: Viscosity development of synthetic polymer during flow through porous media, adapted from Chauveteau, 1986 and Sheng, 2010

The viscosity of a polymer solution can be measured with a rheometer or capillary viscometer.

The rheometer with a Couette system consists of a rotating bob and a fixed cup. The fluid is poured in a cup in which a bob is placed, which causes deliberate shearing due to rotation. The shear rate can be adjusted to cover the viscosity behavior in Newtonian and pseudoplastic regime.

The Ubbelohde viscometer, shown in Figure 2-5, is a capillary-based viscometer that measures the viscosity of a solution with an accuracy of $0.1 \%$, based on Hagen-Poiseuille equation. The polymer solution is poured in the widest capillary of 
the Ubbelohde, until the liquid reaches a level between $C$ and $D$. With a Peleus ball the fluid is sucked into the capillary above the line marked as ' $A$ '. As soon as the fluid drops to ' $A$ ', the time is recorded until the fluid reaches ' $B$ '. The kinematic viscosity can then be determined by the following equation:

$v=K_{U b b} \cdot t$

whereby the constant, $K_{U b b}$, depends on the diameter of the capillary and $t$ is the measured time. The dynamic viscosity, $\eta$, can then be determined by the product of the kinematic viscosity, $v$, and solution density, $\rho$ :

$\eta=v \cdot \rho$

\subsubsection{Polymer Viscoelasticity}

The viscoelasticity of polymers can be studied by the complex shear modulus $G^{*}$ given the Hooke's elasticity law for oscillation, which is defined as the ratio of shear stress and strain:

$G^{*}=\frac{\tau}{\gamma}=G^{\prime}+i G^{\prime \prime}$

$G^{\prime}$ is the storage modulus that represents the real part and provides an indication of how much deformation energy can be stored in the fluid, while the loss modulus, $G^{\prime \prime}$, represents the imaginary part, which corresponds to the viscous response. Equation 2-7 is illustrated in Figure 2-6. By measuring $G^{\prime}$ and $G^{\prime \prime}$ at constant frequency at changing shear stress and strain, conclusions can be drawn about whether it is elastic or viscous behavior that dominates, as shown in Figure 2-7. If $G^{\prime \prime}$ is higher than $G^{\prime}$ in the linear viscoelastic (LVE) range, limited by $\gamma_{L}$, then the polymer solution behaves as an ideally

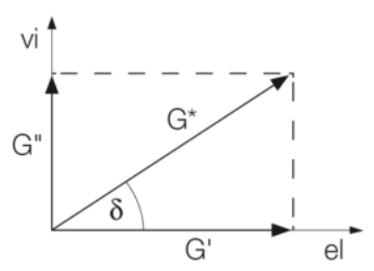

Figure 2-6: Complex shear modulus $G^{*}$ (Goodyer, 2015) viscous fluid having a liquid-like structure. Elastic forces dominate in the opposite case, and the fluid exhibits a gel-like structure. This understanding helps, for instance, to determine the extent to which polymers can store deformation without rupturing the chain (Goodyer, 2015).

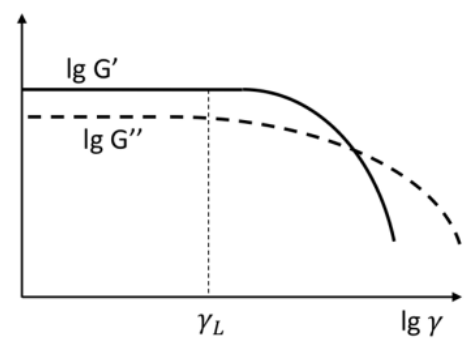

$\mathrm{G}^{\prime}>\mathrm{G}^{\prime \prime}$ in LVE-range

$\rightarrow$ "gel-like structure"

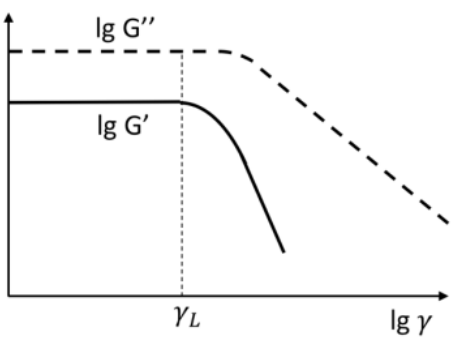

$\mathrm{G}^{\prime \prime}>\mathrm{G}^{\prime}$ in LVE-range

$\rightarrow$ "liquid-like structure"

Figure 2-7: Determination of gel-like and liquid-like structure by measuring storage modulus $G^{\prime}$ and loss modulus $G^{\prime \prime}$ against shear strain (Goodyer, 2015) 
An alternative approach to determine the viscoelasticity of a fluid solution is to employ the screen viscometer, which measures the elongational viscosity from stretched to coiled state.

The screen factor is defined as the ratio of the flow time of polymer solution volume through five 100-mesh screens to the flow time for the same volume of solvent through the screens. The solvent for the used polymer system is surfactant water. It is a quantitative way to determine the degree to which mechanical degradation has been caused by comparing the solutions that have undergone different shearing intensities with each other. Therefore, it is important that for every experiment the same screen viscometer is used as there are differences in the stacking of mesh screens. The principle is similar to the Ubbelohde viscometer. The screen viscometer is shown in Figure 2-8. The liquid here is sucked from the bottom to the top with a Peleus ball, and the time is then measured for the liquid to reach from

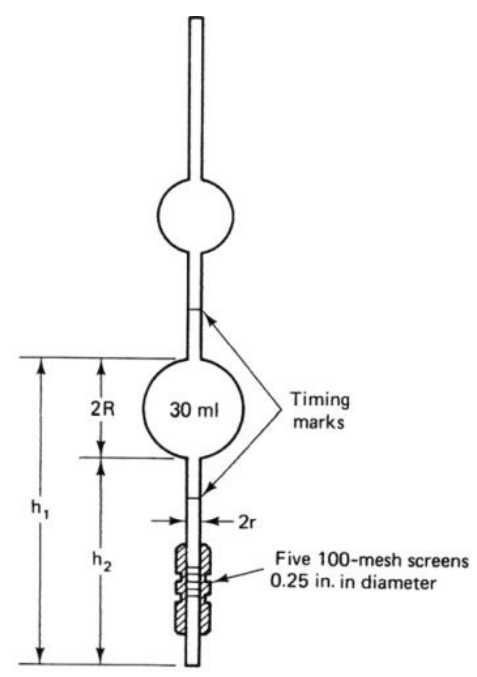

Figure 2-8: Screen viscometer

(Lake, 1989) the first timing mark to the second timing mark (Sheng, 2011).

\subsubsection{Concentration Regimes}

Depending on the concentration of the polymer dissolved in water, the polymer solutions can be categorized in dilute, semi-dilute and concentrated regimes, as shown in Figure 2-9. Thereby, the radius of gyration (indicated with blue circles in Figure 2-9) defines the volume that the polymer occupies. In a dilute system, which occurs at a low concentration of polymer, the polymer chains are separated and do not interact with each other. By increasing the polymer concentration, a concentration threshold, $C^{*}$, is reached, where the concentration regime enters a semi-dilute state. The polymer chains interact with each other and overlap. At the second threshold, $C^{C}$, the polymer concentration is so high that the coils are densely packed, and they entangle strongly with each other. Further, the more the polymer concentration rises, the more the polymer chains entangle and interact with each other (Y. Z. Wang, et al., 2010).

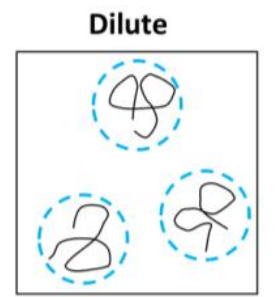

$\mathrm{C}<\mathrm{C}^{*}$

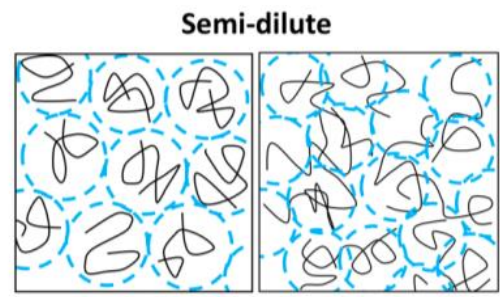

$\mathrm{C}=\mathrm{C}^{*}$ $\mathrm{C}^{*}<\mathrm{C}<\mathrm{C}^{\mathrm{C}}$

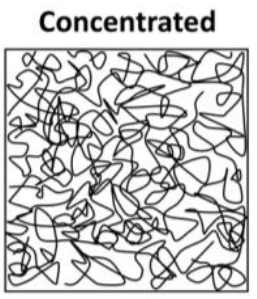

$\mathrm{C}>\mathrm{C}^{\mathrm{C}}$

Figure 2-9: Dilute, semi-dilute and concentration polymer systems, adapted from Y. Z. Wang, et al., 2010

The fluid state can be characterized by measuring the viscosity of the polymer solution. Therefore, three linear trend line regimes occur as shown in Figure 2-10. By intersecting the two neighboring trend lines, the two thresholds, $C^{*}$ and $C^{C}$, can be determined. This allows for distinguishing between dilute, semi-dilute, and concentrated regime in the used polymer system (Chauveteau, 1986; Duffy, 2015; Wang, Li, Xiong, Wang, \& Zhang, 2010). 


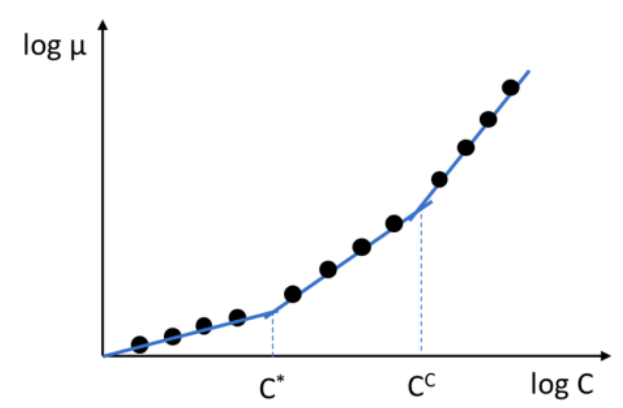

Figure 2-10: Determination of $C^{*}$ and $C^{C}$ (Duffy, 2015)

\subsubsection{Molecular Weight Size}

Synthetic polymers used in EOR generally have a molecular weight size between 2-10 Mega Dalton, whereby synthetic polymers usually display a higher polydispersity index than biological polymers. Thereby, the molecular weight distribution $(M W D)$ is typically positively skewed, which means that a minor amount of chains contributes to the highend of the distribution curve (Sorbie, 1991). An exemplary MWD curve for HPAM-based polymer solution is shown schematically in Figure 2-11.

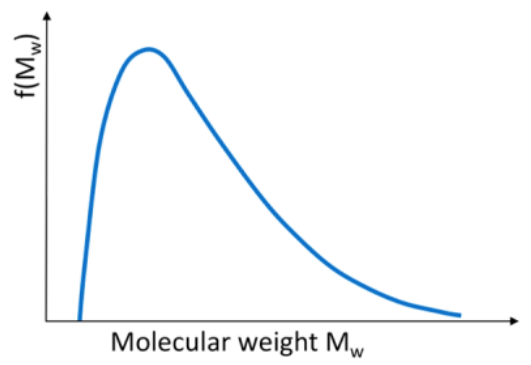

Figure 2-11: Molecular-weight distribution for HPAM-based polymer solutions adapted from Sorbie (1991)

MWD curves can be determined by gel permeation chromatography $(G P C)$ or light scattering, which however cannot always provide accurate results. Especially for high-molecular-weight polymers the $M W D$-measurements becomes increasingly challenging. The average molecular weight, $M_{w, a v}$, can be determined by the Mark-Houwink equation:

$\eta_{\text {int }}=K_{M H} \cdot M_{w, a v}{ }^{a}$

where $\eta_{i n t}$ is the intrinsic viscosity, measuring the solute's contribution to the viscosity of the solution, and $K_{M H}$ and $a$ are the Mark-Houwink parameters that depend on the polymer (Graillat, Pichot, Guyot, \& El Aasser, 1986).

\subsubsection{Polymer Stability}

Polymer solutions are prone to various events of degradation, which cause a loss in viscosity, and thereby a reduction in the sweep efficiency. The degradation sources can have biological, chemical, or mechanical origin. Biological degradation includes the involvement of bacteria, and it is only relevant for biopolymers (Lake, 1989; Sorbie, 1991). Thus, it is not of interest in this study.

Chemical degradation includes all chemical reactions caused by temperature, salinity, oxygen or other components that lead to chemical degradation. In this study, only the impact caused due to salinity has been considered. In high-saline water, the polymer chains form coils due to the presence of divalent ions. Negatively-charged groups form covalent interactions with divalent salt ions, which are much stronger than the hydrogen bonds. As a consequence, the viscosity 
reduces with the increasing salinity due to the increase in coiling. The precipitation with divalent ions is schematically shown in Figure 2-12 (Lake, 1989; Sheng, 2010; Sorbie, 1991).

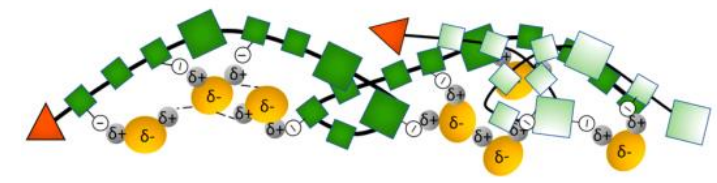

Dissolved in fresh water:

Inter- and intramolecular interactions due to hydrogen bonding

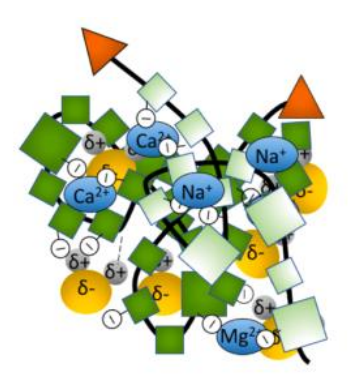

Dissolved in salt water with divalent ions: Precipitation leads to strongly coiled structure

Figure 2-12: Effect of divalent ions on polymer solution in low and high salinity

Mechanical degradation is defined as permanent irreversible loss of viscosity caused by the rupturing of the chain. In a field project, the polymer solution undergoes various mechanical degradation while being in pumps, various surface facilities, wellbore, perforations, and porous media, which are caused by shearing forces with extensional and rotational components. The acrylamide polymers are highly flexible chains, which are, thus, prone to shear degradation. By integrating monomer groups such as acrylate, $\mathrm{n}$-Vinyl-Pyrrolidone ( $n V P)$ and Acrylamido tert-Butyl Sulfonate (ATBS), as shown in Figure 2-13, to the HPAM-chain, the mechanical stability of the polymer can be increased. Thereby, acrylate improves the shear stability by charge repulsion, while $n V P$ and ATBS improve the shear stability by its higher molecular weight, providing chain rigidity. The order of increasing shear stability is acrylamide, acrylate, $n V P$ and ATBS, which also causes a respective increase in the monomer molecular volume (Zaitoun, et al., 2011).

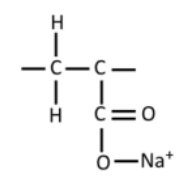

Acrylate (Molecular weight
volume: $114 \AA$ )

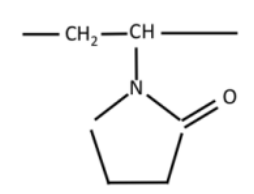

n-Vinyl-Pyrrolidone (Molecular weight volume: $177 \AA$ A)

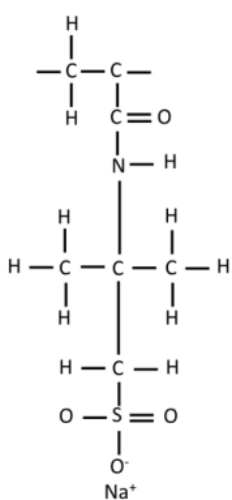

Acrylamido tert-Butyl Sulfonate (ATBS) (Molecular weight volume: $313 \hat{A}$ )

Figure 2-13: Chemical structure of acrylate, nVP and ATBS, adapted from (Zaitoun, et al., 2011)

The mechanical degradation of the polymers can be determined by using the following formula:

$$
\text { Mechanical Degradation }=100 \cdot \frac{\mu_{\text {init }}-\mu_{m}}{\mu_{\text {init }}-\mu_{w}}
$$

where $\mu_{\text {init }}$ is the initial solution viscosity before degradation, $\mu_{m}$ is the viscosity after mechanical degradation, and $\mu_{w}$ is viscosity of the water. Jouenne et al. (2017) suggests that mechanical degradation results from the accumulation of total energy that is obtained during stretching. Further, mechanical degradation was found to occur during abrupt contractions, which are caused by an orifice, porous media, valves, etc., and it does not depend on the geometry or type 
of the mechanical degradation event. This means that mechanical degradation from different origins can be summed up in order to determine the total mechanical degradation. When mechanical degradation occurs, the molecular weight $M_{w}$ distribution was found to move from skewed distribution with increasing mechanical degradation to approach a narrow bell, where the polymer coils do no longer break (Jouenne, Chakibi, \& Levitt, 2017).

\subsection{Polymer Flow in Porous Media}

The flow of polymers through porous media can be described by theoretical approaches that have been introduced in the first section of this chapter. Furthermore, the challenges of polymer flow caused due to polymer retention has also been introduced.

\subsubsection{Theoretical Model of Flow in Porous Media}

The apparent viscosity behavior can be determined by the flow through capillary tubes, which can be seen as analogues to the flow path of the fluid through porous media. The velocity profile in a capillary is parabolic, whereby the maximum velocity occurs
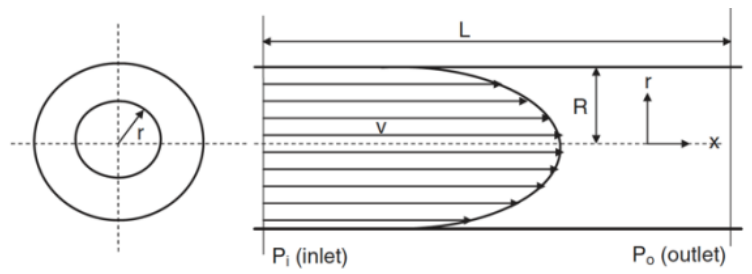

Figure 2-14: Flow profile through capillary (Sheng, 2010) at the middle of the capillary, and the minimum velocity, which is zero, occurs at the wall (see Figure 2-14). In the capillary tubes the following force equilibrium is given by the following equation (Sheng, 2010):

$2 \cdot \pi \cdot r \cdot \Delta L \cdot \tau=\pi \cdot r^{2} \cdot \Delta P$

where $r$ is the radius of the capillary, $\Delta L$ is the length gradient, $\Delta P$ is the pressure gradient of the inlet and outlet of the capillary. The superficial velocity, $u_{D}$, is described by Darcy's law, which states the following:

$$
u_{D}=\phi \cdot v_{i}=\frac{k \cdot \Delta P}{\mu \cdot \Delta L}
$$

where $k$ is the permeability, and $\phi$ the porosity. $k$ is determined by the theory of bundle of tubes, which is given as follows (Sheng, 2010):

$$
k=\frac{\phi \cdot R^{2}}{8}
$$

By inserting Equation 2-2 in Equation 2-10, solving for $\frac{\partial v}{\partial r}$, and integrating the resulting equation over the entire capillary tube, the interstitial flow velocity, $v$, can be determined as follows:

$v_{i}(r)=\left(\frac{\Delta P}{2 \cdot K \cdot \Delta L}\right)^{\frac{1}{n}} \cdot \frac{n}{n+1} R^{\frac{(n+1)}{n}}\left[1-\left(\frac{r}{R}\right)^{\frac{(n+1)}{n}}\right]$

where the average interstitial velocity $\bar{v}$ is given by the following equation:

$\bar{v}_{i}=\left(\frac{\Delta P}{2 \cdot K \cdot \Delta L}\right)^{\frac{1}{n}} \cdot \frac{n}{3 n+1} R^{\frac{(n+1)}{n}}$

Therefore, the average interstitial velocity for a Newtonian fluid, where $K=\mu$ and $n=1$, result in the Hagen-Poiseuille equation, assuming a laminar Newtonian flow with no-slip condition (e.g. $v(R)=0)$ (Sorbie, 1991):

$\bar{v}_{i}=\frac{R^{2} \cdot \Delta P}{8 \cdot \mu \cdot \Delta L}$ 
The force equilibrium can be inserted in the power law model given by Equation 2-2, and the entire equation can then be rearranged to the shear rate with respect to the radius of the capillaries. This gives the following equation:

$\dot{\gamma}(r)=\frac{\partial v_{i}(r)}{\partial r}=-\left(\frac{\Delta P}{2 \cdot K \cdot \Delta L} r\right)^{\frac{1}{n}}$

For simplification, $\dot{\gamma}$ is determined at the maximum shear rate, which is at the maximum radius $r=R$ and then expressed depending on the average interstitial viscosity $\bar{v}$. The maximum shear rate, $\dot{\gamma}(R)$ at the capillary wall results in the following equation:

$\dot{\gamma}(R)=-\left(\frac{\Delta P}{2 \cdot K \cdot \Delta L} R\right)^{\frac{1}{n}}$

By replacing a part of Equation 2-14 with Equation 2-17 and rearranging the shear rate at the capillary wall, $\dot{\gamma}(R)$ can be expressed in dependency of the average interstitial viscosity $\bar{v}$ :

$\dot{\gamma}(R)=\left(\frac{3 n+1}{4 n}\right) \cdot \frac{4 \bar{v}_{i}}{R}$

By inserting Equation 2-11 and Equation 2-12 in Equation 2-18, the average shear rate is determined as follows:

$\bar{\gamma}=\frac{(1+3 n)}{4 n} \cdot \alpha \cdot \frac{4 u_{D}}{\sqrt{8 k \phi}}$

where $\alpha$ is an empirical shift factor that has to be fitted to the experimental data. The shear rate in the capillary tubes can be determined by neglecting the effect of non-Newtonian fluid on the shear rate, assuming Newtonian behavior and laminar. By setting $n=\alpha=\phi=1$ and using Equation 2-19 and replacing the denominator with Equation 2-12, the shear rate in the capillary can be determined as follows (Seright, 1983):

$$
\dot{\gamma}=\frac{4 \cdot u_{D}}{R}=\frac{4 \cdot V}{\pi \cdot R^{3} \cdot t}
$$

The relative apparent viscosity in a capillary can be determined by the ratio of viscosity of the polymer to the viscosity of the solvent, which is water containing the inverter surfactant in the case of an inverse emulsion (Seright, 1983):

$$
\eta_{\text {eff }}=\frac{\mu_{p}}{\mu_{s}}
$$

In an experimental set-up, the relative apparent viscosity in a capillary is calculated by Darcy's law, whose results for the velocity of the surfactant water and polymer is as follows:

$$
[a] \quad u_{\text {solvent }}=\frac{k}{\mu_{s}} \nabla P ; \quad[b] \quad u_{\text {polymer }}=\frac{k}{\mu_{p}} \nabla P
$$

If the pressure and the permeability remain constant, the Equation 2-22a and Equation 2-22b can be equalized, which allows determining the apparent viscosity by the ratio of the solvent velocity and the polymer solution's velocity: $\eta_{\text {eff }}=\frac{\mu_{p}}{\mu_{s}}=\frac{u_{s}}{u_{p}}$ 


\subsubsection{Injectivity Challenges of High-Molecular-Weight Polymer Solutions in Porous Media}

Polymer retention presents a challenge when injecting high-molecular-weight polymer solutions caused by a reduction in the permeability. Ideally, the permeability remains constant during a polymer flood. However, in reality, the permeability reduces due to mechanisms such as (1) adsorption, (2) mechanical trapping, and (3) hydrodynamic retention:

(1) Adsorption is caused by the interaction between polymer and grain surface. It is mainly caused due to physical effects, such as van-der-Waals forces, and it can be influenced by the addition of surfactants and $\mathrm{pH}$ control.

(2) Mechanical trapping occurs especially when the polymer chains are too large to flow through the narrow pore throats and block the pathway for other fluids. Therefore, the high-end of the MWD of the polymer system and the highly coiled chains, formed due to hydrogen bonding as well as precipitation of divalent salt ions, are a major source of mechanical trapping (Sorbie, 1991). As a consequence of mechanical trapping, the risk to cause permanent formation damage, such as unwanted fracking during injection, increases.

(3) Hydrodynamic retention is the least well-studied polymer retention mechanism, and it becomes visible by a change in flow rate. Since it is assumed not to contribute significantly to the total polymer retention in high permeable formations, hydrodynamic retention is neglected in this study (Sheng, 2010; Szabo, 1975).

Polymer retention can be described by the resistance factor, $R F$, and residual resistance factor, $R R F$. The $R F$ is given by the ratio of the mobilities of brine to polymer solution. In an ideal polymer flood, both the permeability and the $R F$ remain constant. Thus, the theoretical $R F$ is given by the ratio of the viscosities of polymer solution to brine, which results as the apparent relative viscosity, $\eta_{e f f}$, of the polymer solution in porous media (Jennings, Rogers, \& West, 1971):

$R F_{\text {theo }}=\frac{\mu_{p}}{\mu_{w}}=\eta_{\text {eff }}$

In practice, the reduction in permeability occurs during the polymer injection. Thereby, $R F$ is given as per the following relation:

$$
R F=\frac{\lambda_{w, \text { before polymer flood }}}{\lambda_{p}}=\frac{\left(\frac{k}{\mu}\right)_{w, \text { before polymer flood }}}{\left(\frac{k}{\mu}\right)_{p}}=\frac{\Delta P_{p}}{\Delta P_{w}}
$$

The $R R F$ shows the degree of polymer retention after polymer flood. It is determined by the ratio of the brine mobility after the polymer flood and the mobility of the polymer solution:

$$
R R F=\frac{\lambda_{w, \text { after polymer flood }}}{\lambda_{p}}=\frac{\left(\frac{k}{\mu}\right)_{w, \text { before polymer flood }}}{\left(\frac{k}{\mu}\right)_{w, \text { after polymer flood }}}=\frac{\Delta P_{w, \text { after polymer flood }}}{\Delta P_{w, \text { before polymer flood }}}
$$

An exemplary profile of a theoretical and actual $R F$ profile in a porous media flood is shown in Figure 2-15 for a sandpack flooding experiment. While the theoretical $R F$ remains constant depending only on the viscosities, the actual $R F$ shows an increase throughout the polymer injection, caused by a reduction in permeability. After the polymer injection, the sand cell is flushed with water, allowing for the determination of the $R R F$, and thereby, the resulting change in permeability.

With the injectivity gradient $I G$, the $R F$ profiles can be easily compared. The $I G$ is determined by the ratio of $R F$ at a certain pore volume, $P V$, to an $R F$ at an early $P V$ : 


$$
I G_{(x+1) P V}=\frac{R F_{(x+1) P V}}{R F_{(x) P V}}
$$

Ideally, the $I G$ should be 1, meaning that there is no increase in $R F$.

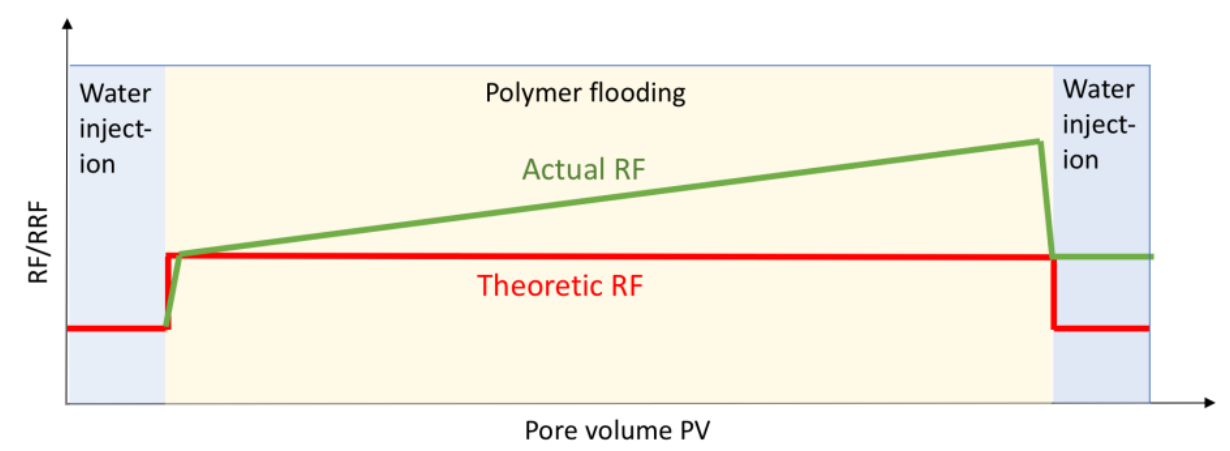

Figure 2-15: Theoretical and actual $R F$ profile in sand cell flood

By deliberately pre-conditioning the polymer solutions, the polymer chains can be de-entangled, bringing them in a stretched state and destroying the high-end of the molecular weight distribution. Pre-conditioning can be achieved by pre-shearing the polymer solution for instance with agitators or flow through contractions. Previous studies of Maerker (1975) and Sorbie (1991) already discovered that mechanical degradation first impacts the high-end of the MWD, narrowing the distribution significantly. The two aims of pre-shearing are illustrated schematically in Figure 2-16.
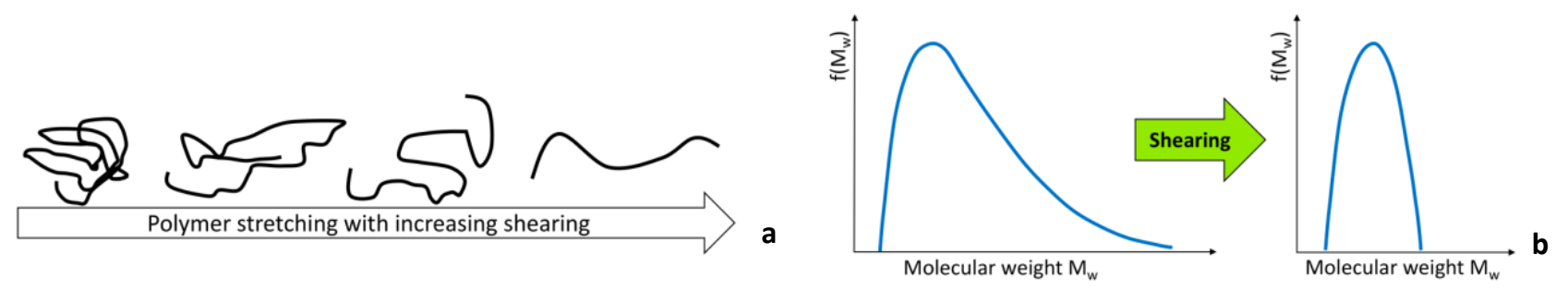

Figure 2-16: Schematic of reasons for pre-conditioning the polymer solution: polymer stretching with increasing shearing [a], narrowing of $M W D[\mathrm{~b}]$

The superimposed volume specific energy input by the agitators can be determined by turbine-power correlations, which is expressed by power number, $N_{p}$, versus Reynolds number for agitators, $R_{e}$. For this, the power input, $P_{N}$, has to be determined first as follows (Sanchez Perez, Rodriguez Procel, \& Casas Lopez, 2006):

$P_{N}=N_{p} \cdot \rho \cdot n_{r}^{3} \cdot d_{i}^{5}$

where $N_{p}$ is the power number, $\rho$ the fluid density, $n_{r}$ the rotational speed, and $d_{i}$ the diameter of the impeller. $N_{p}$ can be expressed by the ratio of a geometry-dependent constant, $C_{g}$, and Reynolds number for agitators, $R_{e} . R_{e}$ is calculated by the Equation 2-29 in the following way (Sanchez Perez, Rodriguez Procel, \& Casas Lopez, 2006):

$R_{e}=\frac{\rho \cdot n_{r} \cdot d_{i}^{2}}{\mu}$

This enables to classify the energy brought into the polymer solution. The imposed volume specific energy input, $E_{v}$, is then determined by the following equation:

$E_{v}=\frac{P_{N} \cdot t}{V}$

where $t$ is the stirring time, and $V$ is the stirred volume. 


\section{Experiments}

This chapter introduces the exerted experiments within this study by covering the materials and methods, the experimental set-up, and the procedure of the bulk and sand-pack flooding tests.

\subsection{Materials and Methods}

The materials and methods section includes the components and preparation of the polymer solution, the applied rheological measurements, and the sand-pack cell used for the flooding tests.

\subsubsection{Preparation of Polymer Solution}

The polymer solution PS-A contains 4,000 ppm active emulsion polymer $E P-A$ and 2,400 ppm active inverter surfactant IS-A. The amount of emulsion polymer and inverter surfactant is constant for all experiments, unless specifically stated otherwise. The components of the polymer solution along with the data about the used amount are given in Table 3-1.

Table 3-1: Components of polymer solution and used amount for polymer solution PS-A

\begin{tabular}{|c|c|c|}
\hline \multirow{2}{*}{ Component } & \multicolumn{2}{|c|}{ Amount } \\
\cline { 2 - 3 } & {$[\mathrm{ppm}]$} & $1038(=1 \mathrm{~L})$ \\
\hline Synthetic sea water SSW-A & & 13.33 \\
\hline Active emulsion polymer $E P-A$ & 4,000 (active) & 3.43 \\
\hline Inverter surfactant IS-A & 2,400 & \\
\hline
\end{tabular}

The composition of synthetic sea water SSW-A is given in Table 3-2.

Table 3-2: Synthetic sea water composition SSW-A with 50,000 ppm TDS

\begin{tabular}{|c|c|c|}
\hline Component & Chemical formula & Salinity [ppm] \\
\hline Sodium & $\mathrm{Na}^{+}$ & 15,121 \\
\hline Potassium & $\mathrm{K}^{+}$ & 1,928 \\
\hline Magnesium & $\mathrm{Mg}^{2+}$ & 649 \\
\hline Calcium & $\mathrm{Ca}^{2+}$ & 3 \\
\hline Strontium & $\mathrm{Sr}^{2+}$ & 27,771 \\
\hline Chloride & $\mathrm{Cl}^{-}$ & 3,700 \\
\hline Sulphate & $\mathrm{SO}_{2}^{-}$ & 153 \\
\hline Hydrogencarbonate & $\mathrm{HCO}_{3}^{-}$ & \\
\hline
\end{tabular}

The polymer solution PS-A is inverted in a 3-liter beaker with an outer diameter of $15 \mathrm{~cm}$. For this, $1038 \mathrm{~g} S S W$ - $A$ are added to the beaker, placed on a magnetic stirrer, which is set to $600 \mathrm{rpm}$. IS-A weighing $3.43 \mathrm{~g}$ is then added to SSW-A. After $1 \mathrm{~min}$ of stirring, the surfactant is distributed in the aqueous phase, and $13.33 \mathrm{~g}$ of $E P-A$ are added. The polymer system is already inverted after a few minutes of stirring, yet to ensure that all components are dissolved, the solution is stirred for $45 \mathrm{~min}$. The polymer solution, that is inverted in the magnetic stirrer, is referred to as the unsheared polymer solution from now on, since no mechanical degradation occurs when stirring with the magnetic stirrer, which was proven by determining the viscosity at different stirring times and observing no viscosity loss.

The polymer solution is pre-conditioned with three different shearing devices at various intensities. Therefore, the unsheared polymer solution is created with the magnetic stirrer and then conditioned with the UltraTurrax or over the 
capillaries. When conditioning with the disperser, the polymer solution is not inverted with a magnetic stirrer beforehand, since the inversion of the solution occurs within a few seconds due to the high turbulence caused by disperser. The schematic set-up is shown in Figure 3-1. The three different shearing devices are introduced in the following section.

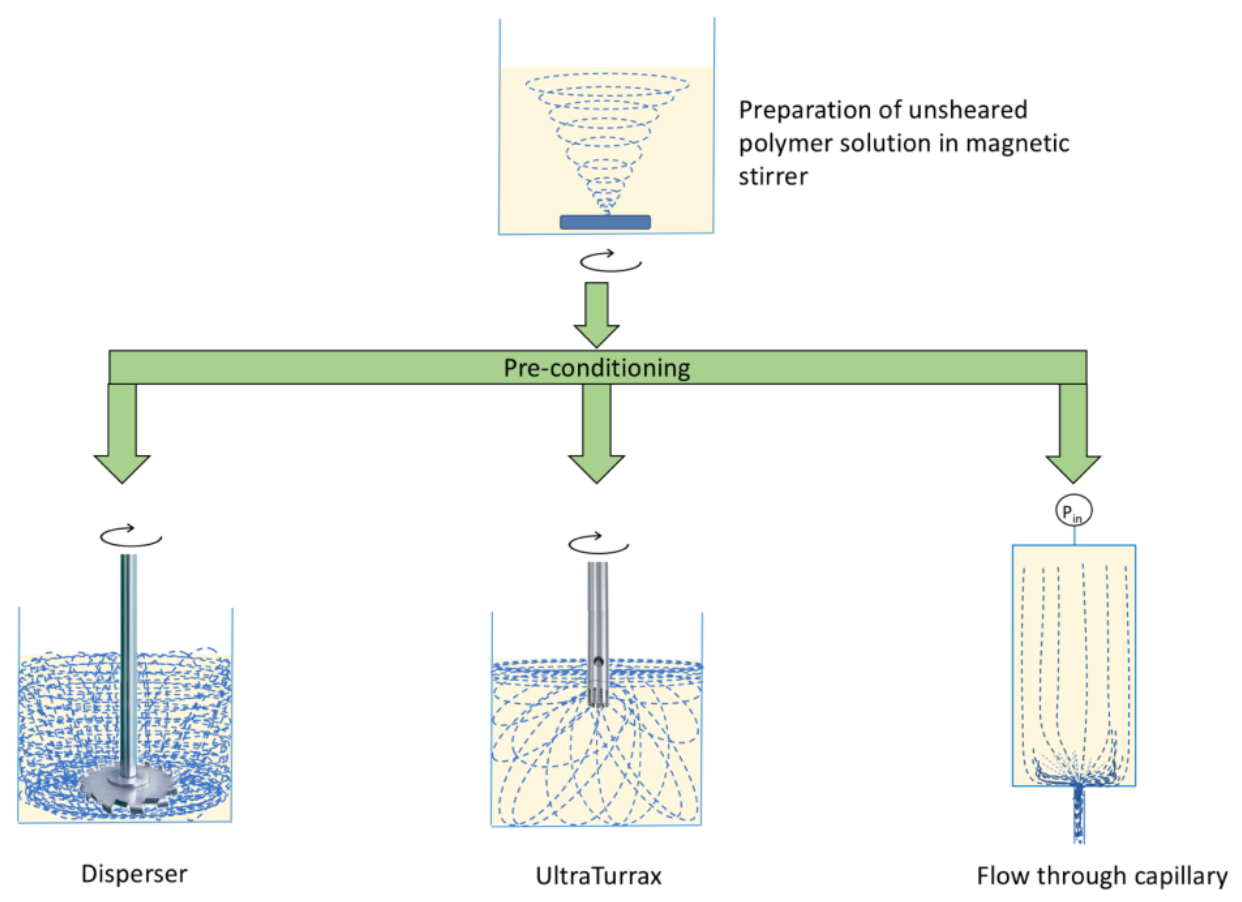

Figure 3-1: Preparation of unsheared polymer solution with magnetic stirrer and conditioned polymer solutions with three shearing devices

\section{Shearing device 1 - Disperser}

The disperser is an agitator that has a toothed circular disk stirrer as shown in Figure 3-2. The disperser model that is used is Buddeberg DS 5, which has a mixer diameter of $7 \mathrm{~cm}$. SSW-A weighing $1038 \mathrm{~g}$ is added to a 3-liter beaker that has an outer diameter of $15 \mathrm{~cm}$. The disperser is positioned $2 \mathrm{~cm}$ from the bottom. IS- $A$ weighing $3.43 \mathrm{~g}$ is added to $S S W$ A. After $1 \mathrm{~min}$ of stirring at a certain shearing intensity, $13.33 \mathrm{~g}$ of EP-A are added and stirred for a certain amount of time. The maximum rotational speed of the agitator is $1750 \mathrm{rpm}$. When shearing is done with the disperser, foam is formed due to the high turbulence. Further experiments are carried out only after the foam disappears.

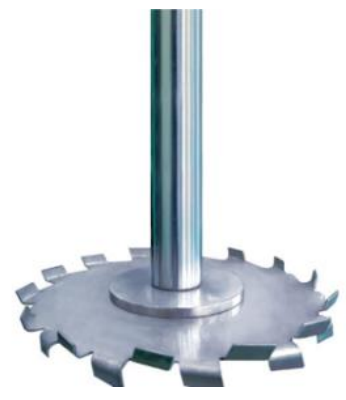

Figure 3-2: Geometry of disperser

\section{Shearing device 2 - Ultra-Turrax}

Before shearing the solution with the Ultra-Turrax, the unsheared polymer solution is inverted in a 3-liter beaker in a magnetic stirrer for $45 \mathrm{~min}$ as has been described above. The unsheared polymer solution is sheared with Ultra-Turrax $\mathrm{S}$ $25 \mathrm{~N}-18 \mathrm{G}$ provided by IKA, which can reach a rotational speed of $20,000 \mathrm{rpm}$. The polymer solution is forced through the gaps of the rotating rotor in the shear gap between the rotor and stator, where high turbulence occurs. The UltraTurrax S $25 \mathrm{~N}-18 \mathrm{G}$ is, hereinafter, simply referred to as turrax. 

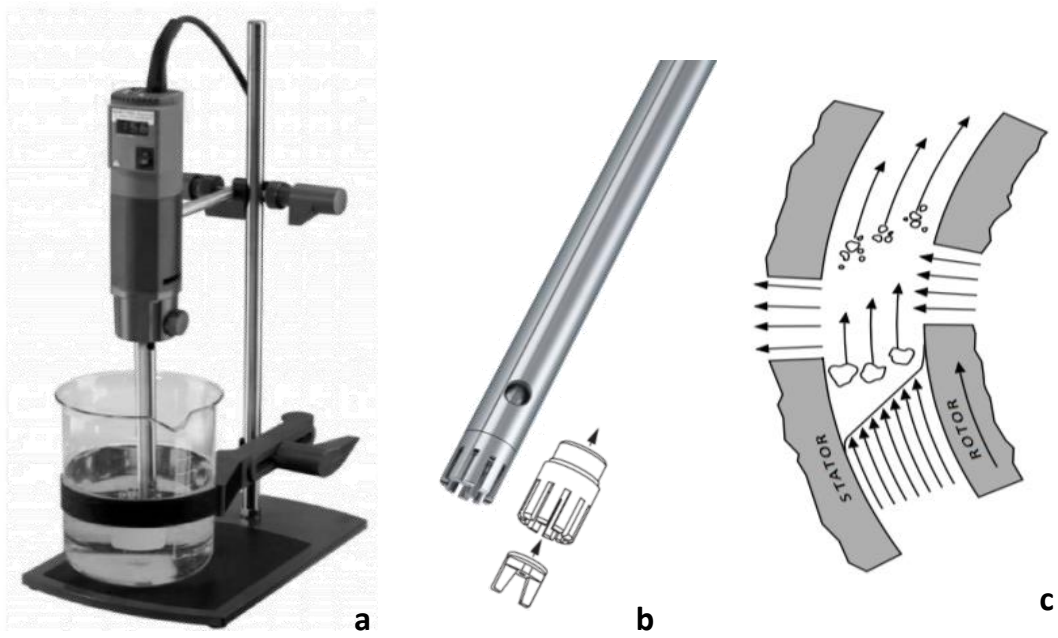

Figure 3-3: Ultra-Turrax S 20 N - 18G assembled in dispersion tool [a], turrax [b], schematic representation of shearing between stator and rotor [b] (IKA)

\section{Shearing device 3 - Capillaries}

The experimental set-up of the pressure-driven flow through a capillary consists of a steel cylinder with a volume of $300 \mathrm{ml}$ and an attached capillary that has an inner diameter of $1 \mathrm{~mm}$ and varying lengths of 3, 10, and $20 \mathrm{~cm}$ at the end of the cylinder. At the top of the cylinder, a pressure gauge is installed, which applies pressures between 1 to 20 bar. The unsheared polymer solutions with the same amount of SSW-A,IS-A, and EP-A is inverted in a magnetic stirrer at $600 \mathrm{rpm}$ for $45 \mathrm{~min}$, as described above. Unsheared polymer solution of $250 \mathrm{ml}$ volume is then successively filled in a steel cylinder and forced through the capillary at a constant pressure. At the exit of the capillary, the outcoming polymer solution is collected in a beaker, placed on a balance, thereby measuring the volumetric flow. The experimental set-up is shown in Figure 3-4. The same experiment is exerted for surfactant water, which is considered to be the solvent of the polymer system. It is prepared by stirring $1038 \mathrm{~g} S S W-A$ and $3.43 \mathrm{~g} I S-A$ for $1 \mathrm{~min}$ with the magnetic stirrer at $600 \mathrm{rpm}$. The capillary flow velocity of the polymer solution and solvent at
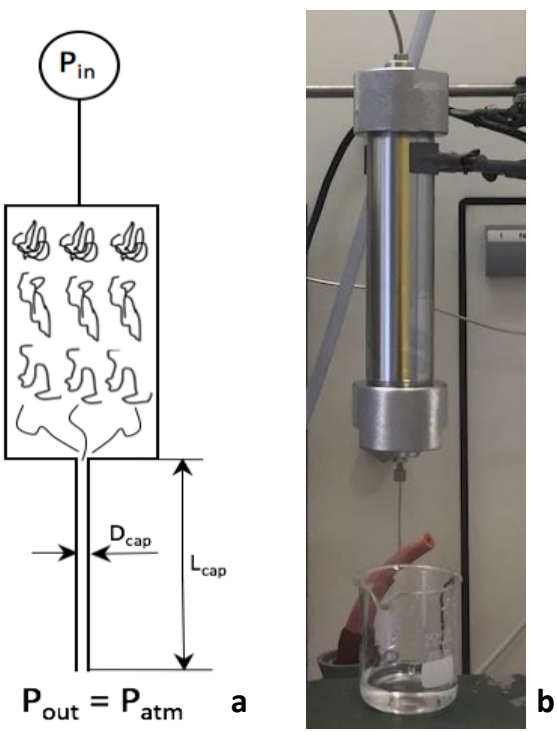

Figure 3-4: Schematic representation of experimental set-up of capillary tubes [a], experimental set-up of capillary tubes [b] different pressures is determined by dividing the volumetric flow by the cross-sectional area of the capillary tube. The shear rate and the relative apparent velocity of the capillary tube are then calculated with Equation 2-20 and Equation 223 respectively.

\subsubsection{Rheological Measurements}

The differently sheared polymer solutions are characterized by determining the rheological properties including viscosity and viscoelasticity, as well as average molecular weight and $\mathrm{pH}$.

\section{a) Viscosity and Viscoelasticity}

The viscosity is determined with an Ubbelohde viscometer that is placed in a $30^{\circ} \mathrm{C}$ water bath. The polymer solution is tempered for $10 \mathrm{~min}$ in the Ubbelohde. The Ubbelohde that was used has the type number 50113 Ic that has an internal 
capillary tube diameter of $0.01 \mathrm{~mm}$, and it can be used to determine a kinematic viscosity $v$ between 3 to $30 \mathrm{~mm}^{2} / \mathrm{s}$. The constant, $K_{U b b}$, is 0.03 for the used Ubbelohde.

Further, the viscosity against shear rate is measured with the Anton Paar rheometer MCR 302 equipped with a concentric cylinder (B-CC27) and measuring cup (C-CC27/SS/Air), as well as the R/S+ Brookfield with double-gap geometry. All viscosity measurements are done at $30{ }^{\circ} \mathrm{C}$. The complex shear modulus is measured with the Anton Paar rheometer MCR302.

The screen factor is determined as described in section 2.3.2 of this thesis. The solvent is the surfactant water, which is prepared by stirring $1038 \mathrm{~g} S S W-A$ and $3.43 \mathrm{~g} / S-A$ for $1 \mathrm{~min}$ with the magnetic stirrer at $600 \mathrm{rpm}$.

\section{b) Average Molecular Weight}

In order to determine the average molecular weight, the polymer needs to be extracted from the polymer solution. The separation of the polymer from the PS-A is shown in Figure 3-5. The polymer is separated by pouring $50 \mathrm{ml}$ of the $P S-A$ in a cup filled with $300 \mathrm{ml}$ ethanol that is placed on a magnetic stirrer. Upon continuous stirring, the polymer precipitates (see Figure 3-5a). The precipitated polymer is filtered with a suction filter that is attached to a vacuum pump (see Figure 3-5b). By pouring ethanol over the separated polymer in the suction filter and turning on the vacuum pump, the remnants are separated from the polymer. This process is repeated three times. The separated polymer is then dried for 24 hours in a drying oven. The dried polymer is shown in Figure 3-5c. The polymer is then dissolved in distilled water, having $0.5 \%$ polymer content (see Figure 3-5d). Dilution series at 3, 2, 1, 0.75, $0.5 \mathrm{~g} / \mathrm{L}$ polymer concentration of the $0.5 \%$ polymer solution are created, and the viscosities are measured for the dilution series with the Ubbelohde viscometer and R/S+ Brookfield rheometer. By plotting the viscosities of the polymer solution at the different concentrations, the intrinsic viscosity can be determined by extrapolating the resulting trend to zero concentration. The Mark-Houwink constants, $K$ and $a$, are chosen to be $6.31 \mathrm{E}-03$ and 0.8 , which corresponds to water as solvent and polyacrylamide as polymer (Graillat, Pichot, Guyot, \& El Aasser, 1986).
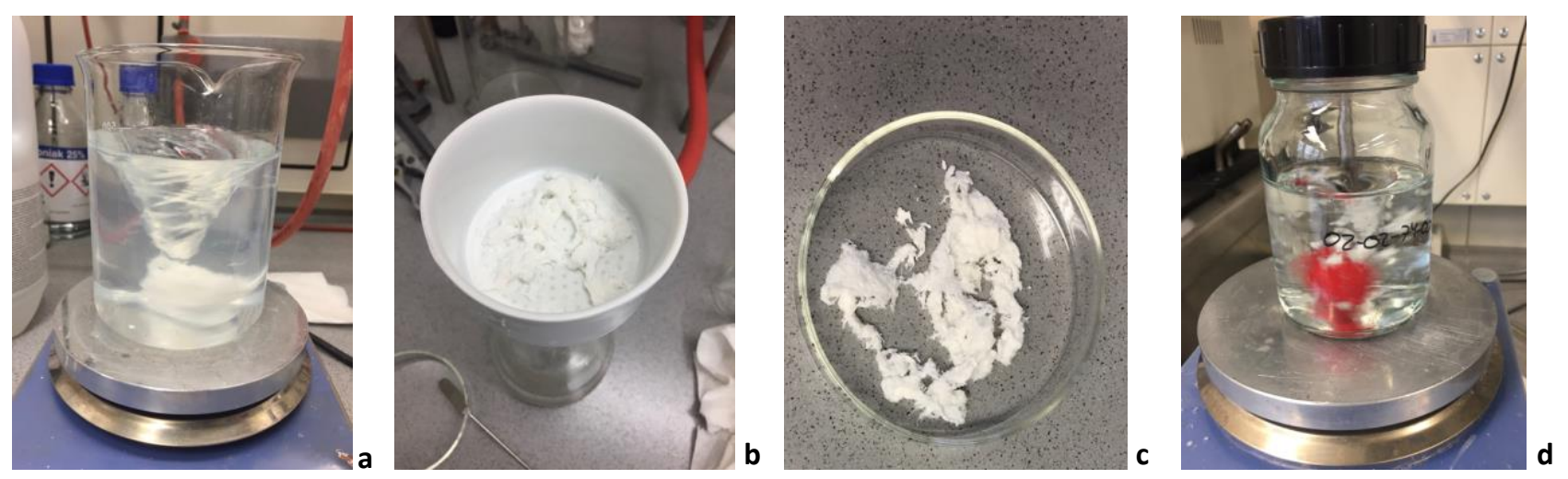

Figure 3-5: Polymer separation in ethanol [a], washing separated polymer with suction filter [b], dried polymer [c], dissolved in distilled water $[d]$

\section{c) $\mathrm{pH}$ Measurement}

The $\mathrm{pH}$ is measured for all solutions. The device used for measuring $\mathrm{pH}$ is Portamess ${ }^{\circledR} 911 \mathrm{pH}$ from Knick with an InLab Reach Pt1000-225 electrode that contains an integrated temperature sensor. 


\subsubsection{Sand-pack Cell}

The sand cell, used for the flooding test, has a cylindrical shape, and it is made out of SS 316 steel (see Figure 3-6). The sand cell has an inner diameter of $1.52 \mathrm{~cm}$ and a length of $7.5 \mathrm{~cm}$, which provides a volume of $13.6 \mathrm{~cm}^{3}$. At the bottom of the sand cell a $30 \mu \mathrm{m}$ square mesh filter, and a coarser filter are installed. The

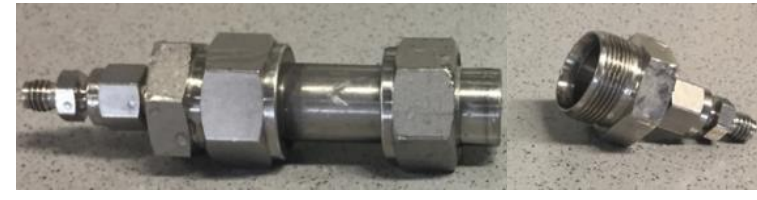

Figure 3-6: Sand cell for injectivity test sand that is used is a quartz sand with a particle size of 0-125 $\mu \mathrm{m}$. The average particle size is $80 \mu \mathrm{m}$. Quartz sand weighing $20 \mathrm{~g}$ is fitted into the sand cell under continuous gentle hammering in the vertical and the horizontal direction, leaving a $6 \mathrm{~mm}$ air gap at the top of the sand cell. The pore volume is determined by the difference in the bulk volume and the volume occupied by the sand, and it results as $6.5 \mathrm{~cm}^{3}$. By dividing the pore volume to the bulk volume, a porosity of $46 \%$ can be determined.

\subsection{Experimental Set-up}

The experimental set-up includes a filtration test, the evacuation process of sand cell and the sand-pack flooding apparatus.

\subsubsection{Filtration Test}

A filtration test is performed in order to characterize the injectivity of the polymer solution, which will be injected into porous media. The polymer solution is poured in a 1-liter steel cylinder that has a pressure gauge on the top, having a pressure of 1 bar. The bottom of the cylinder contains a 1-micron polycarbonate membrane, through which the solution is filtered. At the outlet of the cylinder a valve is installed, allowing the outcoming polymer solution to be collected in a beaker. The beaker is placed on a balance in order to determine the volumetric flow. The set-up is shown in Figure 3-7. The entire solution is filtered, not only to determine the $F R$, but also to avoid pore blocking that is caused by undissolved molecules or impurities. The following relation has been used to determine the FR (Sheng, 2011):

$$
F R=\frac{t_{200 \mathrm{ml}}-t_{180 \mathrm{ml}}}{t_{80 \mathrm{ml}}-t_{60 \mathrm{ml}}}
$$

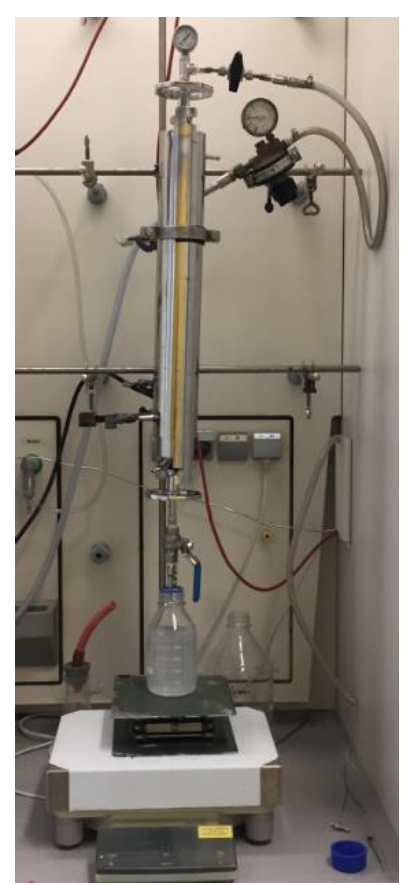

Figure 3-7: Filtration test set-up

where the subscript represents the cumulative volume of the filtrate. An $F R$ of 1 to 1.2 is required to ensure fluid injectivity (Sheng, 2010).

\subsubsection{Evacuation of Sand Cell}

The sand cell is evacuated before the injection test. For this, the exit of the sand cell is attached to a vacuum pump, which allows for the evacuation of the cell. The entrance of the cell is attached to a tube that is connected to a tank filled with SSW-A. While vacuum is maintained, the valve of the water carrying tube at the top of the sand is closed. The sand cell is evacuated for $10 \mathrm{~min}$. After evacuation, the vacuum pump is turned off, and the valve of the water tube is opened so that the sand cell is flooded with SSW-A for $10 \mathrm{~min}$. The experimental set-up is shown in Figure 3-8a. 


\subsubsection{Sand-pack Flooding Tests}

The experimental set-up of the sand-pack flooding test is shown in Figure 3-8b. The previously described sand cell is installed into the flooding set-up. A pressure sensor type A-10 from WIKA with an accuracy of $0.5 \%$ is installed above the sand cell, which allows pressure measurements from 0 to 4 bar. SSW-A is pumped through the entrance tube of the setup. The use of a two-way valve after the pump, allows SSW-A to either flow directly into the sand-pack or in an equalizing tank filled with a spacer-oil. The equalizing oil tank is connected to an equalizing polymer tank, which allows the polymer solution to flow in the sand cell without being directly pumped. This ensures that no mechanical degradation of the polymer occurs by pumping.
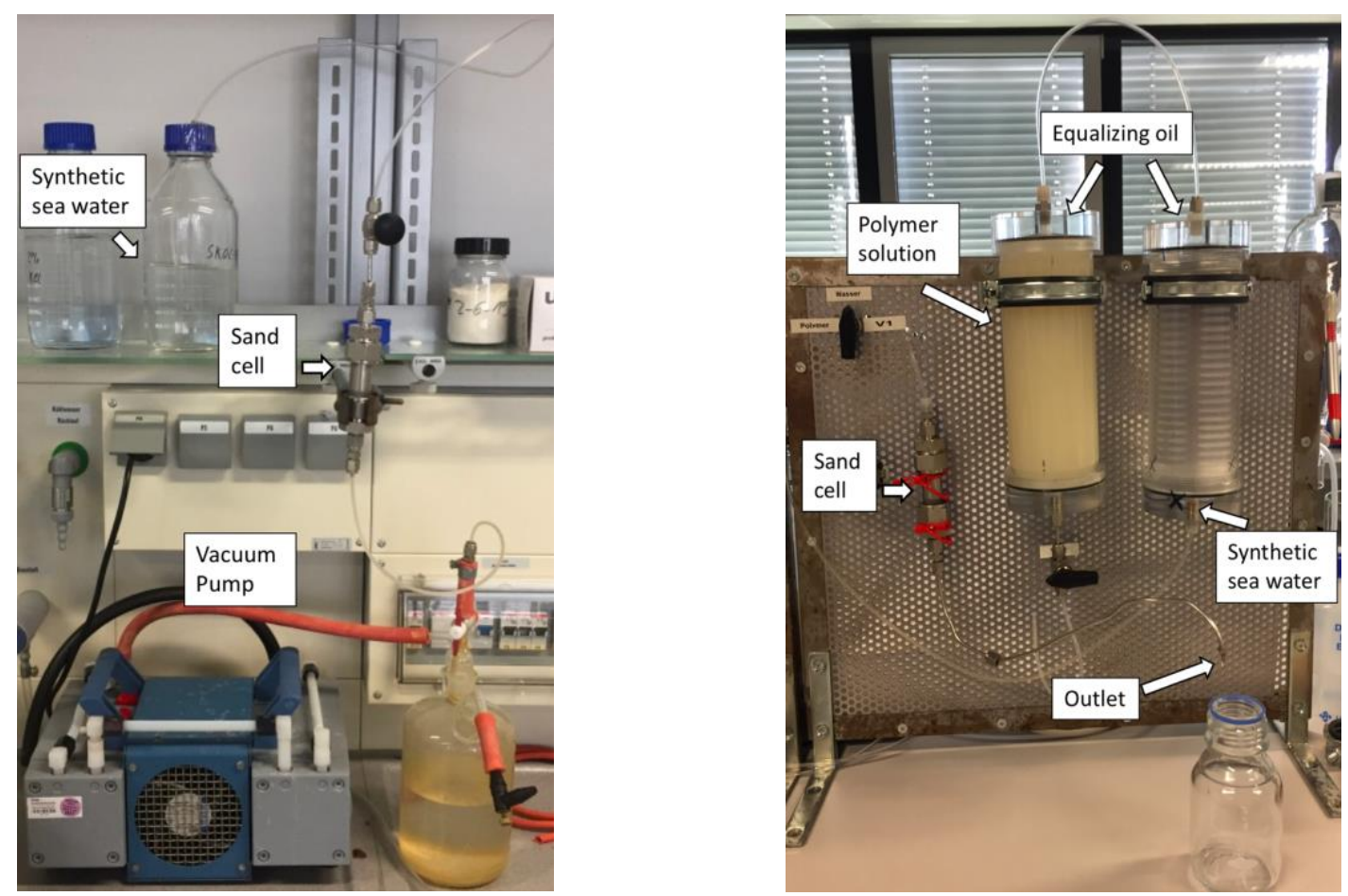

Figure 3-8: Vacuum pump attached to sand cell [a] and experimental set-up of sand-pack flooding test [b]

\subsection{Experimental Procedure}

The experiments are divided into bulk experiments and sand-pack flooding experiments. With the bulk experiments the rheological response of the polymer solution to pre-conditioning is studied, while the flooding experiments aim to investigate on the flow behavior of the polymer solution in porous media.

\subsubsection{Bulk Experiments}

For the bulk experiments, the polymer solution is sheared at different intensities with the disperser, turrax and capillaries. Subsequently, the rheology including viscosity, viscoelasticity, screen factor, average molecular weight and $\mathrm{pH}$ are measured. The exerted shearing intensities for the bulk experiments are summarized in Table 3-3. 
Table 3-3: Shearing intensities per shearing device for bulk experiments

\begin{tabular}{|c|c|}
\hline Shearing device & Shearing intensity \\
\hline Disperser & $500,750,1000,1250,1500$ and $1750 \mathrm{rpm}$ for both 30 and $60 \mathrm{~min}$ \\
\hline Turrax & $5,000,10,000$, and $20,000 \mathrm{rpm}$ for $1,3.25,5,7.75$, and $10 \mathrm{~min}$ \\
\hline Capillary & $\begin{array}{r}\text { Flow under constant pressure of } 1,2,3,4,5,10,20 \text { bar through capillaries } \\
\text { with the lengths of } 3,10,20 \mathrm{~cm}\end{array}$ \\
\hline
\end{tabular}

\subsubsection{Sand-pack Flooding Tests}

The sand injection consists out of five major steps that include the following:

(1) Preparation and conditioning of the polymer solution

(2) Performance of filtration test

(3) Determination of the rheology of the degassed polymer solution before injection

(4) Performance of the sand-pack flooding test

(5) Determination of the rheology of the polymer solution after injection

This procedure is shown schematically in Figure 3-9. The details of the set-up are explained below.

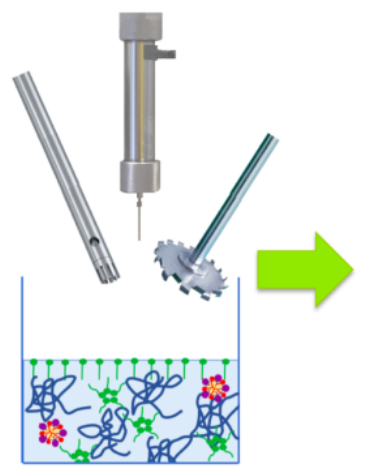

1. Invert polymer solution and pre-shearing of polymer solution PS-A

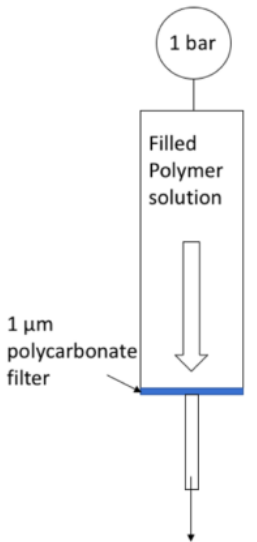

2. Filtration test $F R=\frac{t_{200 m l}-t_{180 m l}}{t_{80 m l}-t_{60 m l}}$
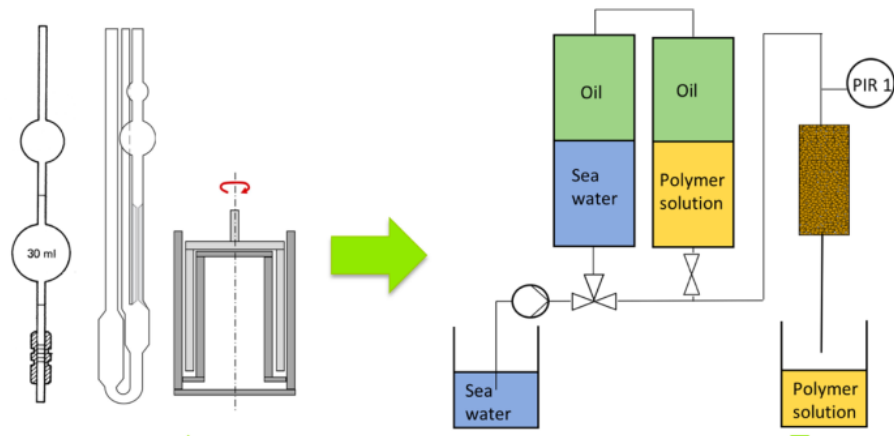

3./5. Rheology determination (screen, Ubbelohde, rheometer) before and after injection

Figure 3-9: Schematic representation of the procedure of injection test

The injection rate of the polymer solution into porous media is given by the client at $0.5 \mathrm{ml} / \mathrm{min}$. The procedure of the sand-pack flooding test is summarized in Table 3-4 and schematically shown in Figure 3-10. In the beginning of the experiment, SSW-A flows directly through the sand cell. The sand cell is flushed for $30 \mathrm{~min}$ at $3 \mathrm{ml} / \mathrm{min}$ followed by 30 $\min$ of flushing at $0.5 \mathrm{ml} / \mathrm{min}$ with SSW-A. The measurement of pressure at different flow rates and the chosen time period of $30 \mathrm{~min}$ increases the accuracy in the permeability estimation using Darcy's law. After SSW-A flooding, the valve at the entrance of the equalizing oil tank is changed to the direction of the tank, and the valve at the exit of the equalizing polymer tank is opened. In this manner, SSW-A is pumped in the first equalizing tank, whereby the oil stays on top the water due to the difference in density. With the inflow of water in the tank, the oil flows in the equalizing polymer tank, pushing the polymer in the tube towards the sand cell. The polymer begins to flood the sand-pack. After the polymer flooding is completed, the valves are switched back to SSW-A flooding for 1 hour, in order to determine a stable RRF value. 
Table 3-4: Procedure of sand-pack flooding test

\begin{tabular}{|c|c|c|c|c|}
\hline Sequence & \multirow{2}{*}{ Injected fluid } & Injection rate & Injection time & PV \\
\cline { 3 - 5 } & & {$[\mathrm{ml} / \mathrm{min}]$} & {$[\mathrm{min}]$} & {$[-]$} \\
\hline 1 & \multirow{2}{*}{ Synthetic sea water $\boldsymbol{S S W}-\boldsymbol{A}$} & 3 & 30 & $\sim 14$ \\
\cline { 3 - 5 } & & 0.5 & 30 & $\sim 2$ \\
\hline 2 & Polymer solution $\boldsymbol{P S}-\boldsymbol{A}$ & 0.5 & Over night & $\sim 66$ \\
\hline 3 & Synthetic sea water $\boldsymbol{S S W}-\boldsymbol{A}$ & 0.5 & 60 & $\sim 2$ \\
\hline 4
\end{tabular}

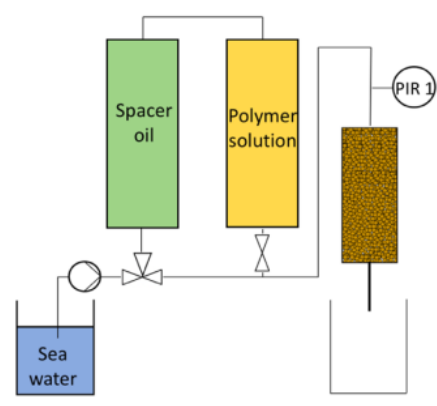

Step 0

Before starting experiment

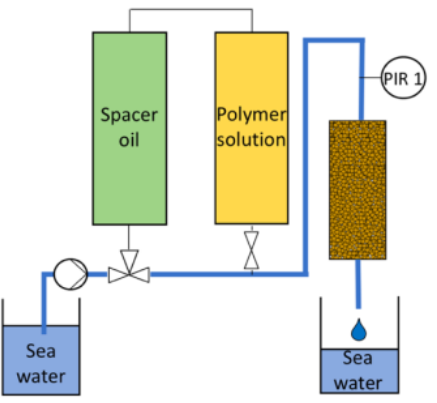

Steps $1 \& 2$

Sea water Injection at 0.5 and $3 \mathrm{ml} / \mathrm{min}$

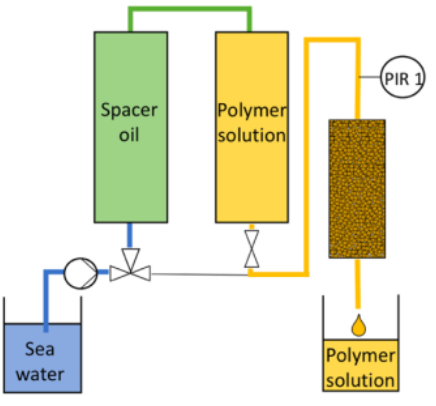

Step 3

Polymer Injection at $0.5 \mathrm{ml} / \mathrm{min}$

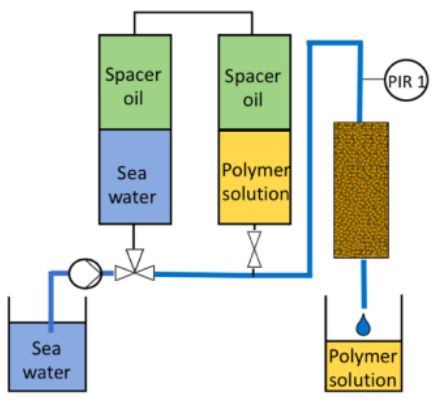

Step 4

Sea water injection after polymer injection at $0.5 \mathrm{ml} / \mathrm{min}$

Figure 3-10: Schematic set-up of sand-pack flooding test at different stages of injection, referring to Table 3-4 


\section{Results and Discussion}

In this chapter, the results of the bulk and sand-pack flooding tests are presented. The overview of the experiments is given in Table 4-1. Bulk experiments of the unsheared and sheared polymer solution were performed in order to gain an understanding of the influence of conditioning on the polymer system before polymer flooding in porous media. The rheological fluid characterization of the unsheared polymer solution is presented in section 4.1. Similar data for the sheared polymer solutions are discussed in section 4.2. The flow of unsheared and sheared polymer solutions into sandpacks is discussed in section 4.3.

Table 4-1: Overview of exerted experiments

\begin{tabular}{|c|c|c|c|}
\hline \multicolumn{2}{|c|}{ Experiments } & \multirow{2}{*}{$\begin{array}{l}\text { Section } \\
4.1 .1\end{array}$} & \multirow{2}{*}{$\begin{array}{l}\text { Overview of experiments in sections } \\
\text { Rheological overview } \\
\text { Shows } \mathrm{pH} \text {, screen factor, viscosity from Ubbelohde of the unsheared solution }\end{array}$} \\
\hline \multirow{9}{*}{ 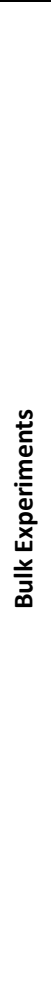 } & \multirow{4}{*}{ 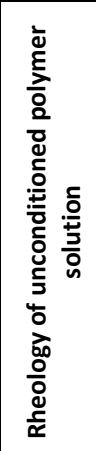 } & & \\
\hline & & 4.1 .2 & $\begin{array}{l}\text { Critical polymer concentration } \\
\text { Shows the viscosity at different polymer concentrations of the unsheared solution, which enables the establishment of } \\
\text { concentration regimes }\end{array}$ \\
\hline & & 4.1 .3 & $\begin{array}{l}\text { Polymer viscosity } \\
\text { Shows the viscosity measured by the rheometer and Ubbelohde, as well as the corresponding shape memory of the } \\
\text { unsheared solution }\end{array}$ \\
\hline & & 4.1 .4 & $\begin{array}{l}\text { Polymer viscoelasticity } \\
\text { Shows the storage and loss modulus against shear stress of the unsheared solution, which gives evidence about } \\
\text { deformability }\end{array}$ \\
\hline & \multirow{5}{*}{ 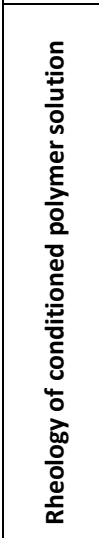 } & 4.2 .1 & $\begin{array}{l}\text { Device-dependent rheological response to pre-conditioning } \\
\text { Shows the screen factor and viscosity measured by Ubbelohde after shearing the polymer solution with each shearing } \\
\text { device and establishing correlations between the different shear origins }\end{array}$ \\
\hline & & 4.2 .2 & $\begin{array}{l}\text { Critical polymer concentration } \\
\text { Shows the viscosity at different polymer concentrations of differently sheared solutions, which enables the } \\
\text { establishment of concentration regimes }\end{array}$ \\
\hline & & 4.2 .3 & $\begin{array}{l}\text { Polymer viscosity } \\
\text { Shows the viscosity measured by the rheometer and Ubbelohde of differently sheared solutions }\end{array}$ \\
\hline & & 4.2 .4 & $\begin{array}{l}\text { Polymer viscoelasticity } \\
\text { Shows the storage and loss modulus against shear stress of differently sheared solutions, which gives evidence about } \\
\text { deformability }\end{array}$ \\
\hline & & 4.2 .5 & $\begin{array}{l}\mathrm{pH} \\
\text { Shows } \mathrm{pH} \text { of polymer solutions, sheared with different shearing devices }\end{array}$ \\
\hline \multirow{4}{*}{\multicolumn{2}{|c|}{ 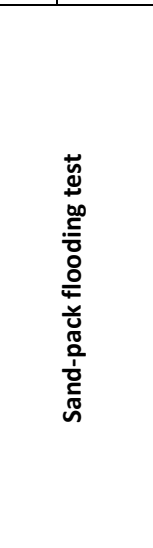 }} & 4.3 .1 & $\begin{array}{l}\text { Injection of unconditioned polymer solution } \\
-\quad \text { Shows injection results of unsheared polymer solution, including } \mathrm{pH} \text {, viscosity from rheometer and Ubbelohde and } \\
\text { screen factor } \\
\text { - } \quad \text { Compares injection results of unsheared polymer solution with higher and lower surfactant concentration }\end{array}$ \\
\hline & & 4.3 .2 & $\begin{array}{l}\text { Device-specific impact of pre-conditioning on injectivity } \\
\text { Shows injection results of each disperser-, turrax-, capillary and disperser-turrax-sheared solutions }\end{array}$ \\
\hline & & 4.3 .3 & $\begin{array}{l}\text { Device-independent impact of pre-conditioning on injectivity } \\
-\quad \text { Compares differently sheared polymer solutions } \\
-\quad \text { Shows mechanical degradation in sand-pack } \\
\text { - Shows of polymer retention by determination of permeability reduction over time using Darcy's law and by } \\
\text { determination of adsorption via nitrogen content }\end{array}$ \\
\hline & & 4.3 .4 & $\begin{array}{l}\text { Impact of polymer and surfactant concentration on pre-conditioned polymer solution } \\
-\quad \text { Observes viscosity influence of sheared polymer solution by increasing the polymer concentration } \\
-\quad \text { Observes surfactant influence of unsheared and sheared polymer solution by changing surfactant concentration }\end{array}$ \\
\hline
\end{tabular}

\subsection{Rheological Fluid Characterization of Unconditioned Polymer Solution}

This section covers the rheological fluid characterization of the unsheared polymer solution, which provides the base value to the differently sheared polymer solutions. This allows to draw conclusions of the beneficial impact of preconditioning to the polymer solution before injecting. 


\subsubsection{Rheological Overview}

The viscosity of the unsheared polymer solution is determined with the Ubbelohde viscometer, and it is found to be $12.5 \pm 0.6 \mathrm{mPas}$. The screen factor is determined with the screen viscometer, and it is found to be $19.0 \pm 0.9$. This has been calculated by acquiring the ratio of flow time of the polymer solution $(=142.8 \pm 7.0 \mathrm{sec})$ and solvent time $(=7.5 \pm$ $0.3 \mathrm{sec})$. For the $\mathrm{pH}$, a value of $7.3 \pm 0.3$ is measured.

\subsubsection{Critical Polymer Concentration}

The polymer concentration regime was determined by measuring the viscosities at different polymer concentration, as described in section 2.3.3. The results of the unsheared polymer solution are shown in Figure 4-1. Three linear trend lines were matched to the three concentration regimes, allowing for the determination of $C^{*}$ at 1,200 $\pm 10 \mathrm{ppm}$ and $C^{C}$ at $3,620 \pm 10 \mathrm{ppm}$. At a polymer concentration of $4,000 \mathrm{ppm}$, the polymer solution is seen to just passed the threshold of the critical concentration $C^{C}$, and it is in concentrated regime. This means that the polymer chains interact with each other and entangle.

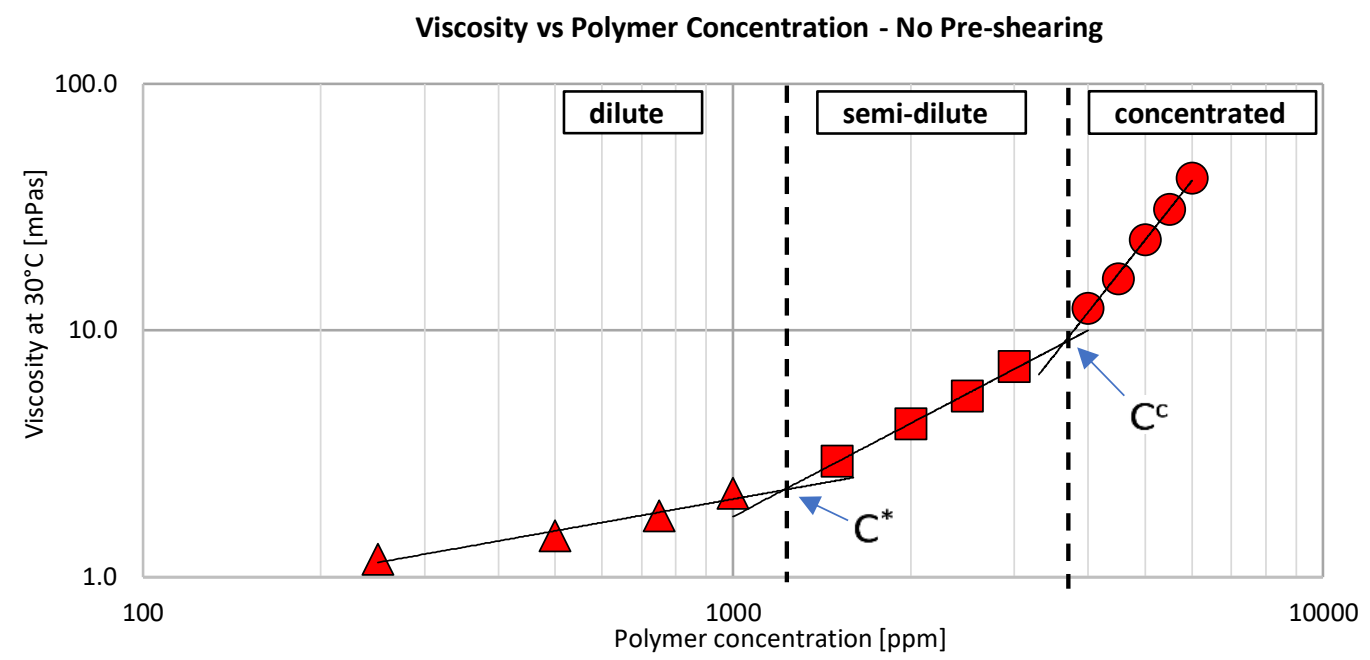

Figure 4-1: Viscosity measured by Ubbelohde vs polymer concentration - no pre-shearing; only inversion with magnetic stirrer with 2,400 ppm inverter surfactant IS-A

\subsubsection{Polymer Viscosity}

The viscosity of the unsheared polymer solution was also determined with the Anton Paar rheometer, from a low shear rate to a high shear rate. Therefore, the shape memory of the unsheared polymer solution was investigated at changing shear rate. This measurement ensures that the polymer solution keeps its viscosifying power independent of the shear history. The shear rate was changed at intervals, running from low to high shear rate and back again. The shear rate was run twice from low to high shear rate and back again, so that four curves were evolved, which matched each other, as shown in Figure 4-2. This means that the viscosity is maintained with changing shear rates. In practice, the polymer solution is exposed to various changes in shear rate. The viscosity curves show shear thinning behavior. Newtonian behavior is indicated at low shear rates, however it is difficult to determine due to the lack of viscosity data at shear rates smaller than $1 \mathrm{~s}^{-1}$. The viscosity measured by the Ubbelohde is also shown in Figure 4-2, whereby the shear rate is calculated with Equation 2-20. A slight discrepancy of $<5 \%$ between the viscosities measured by the Ubbelohde and the rheometer can be observed, due to measurements errors. 


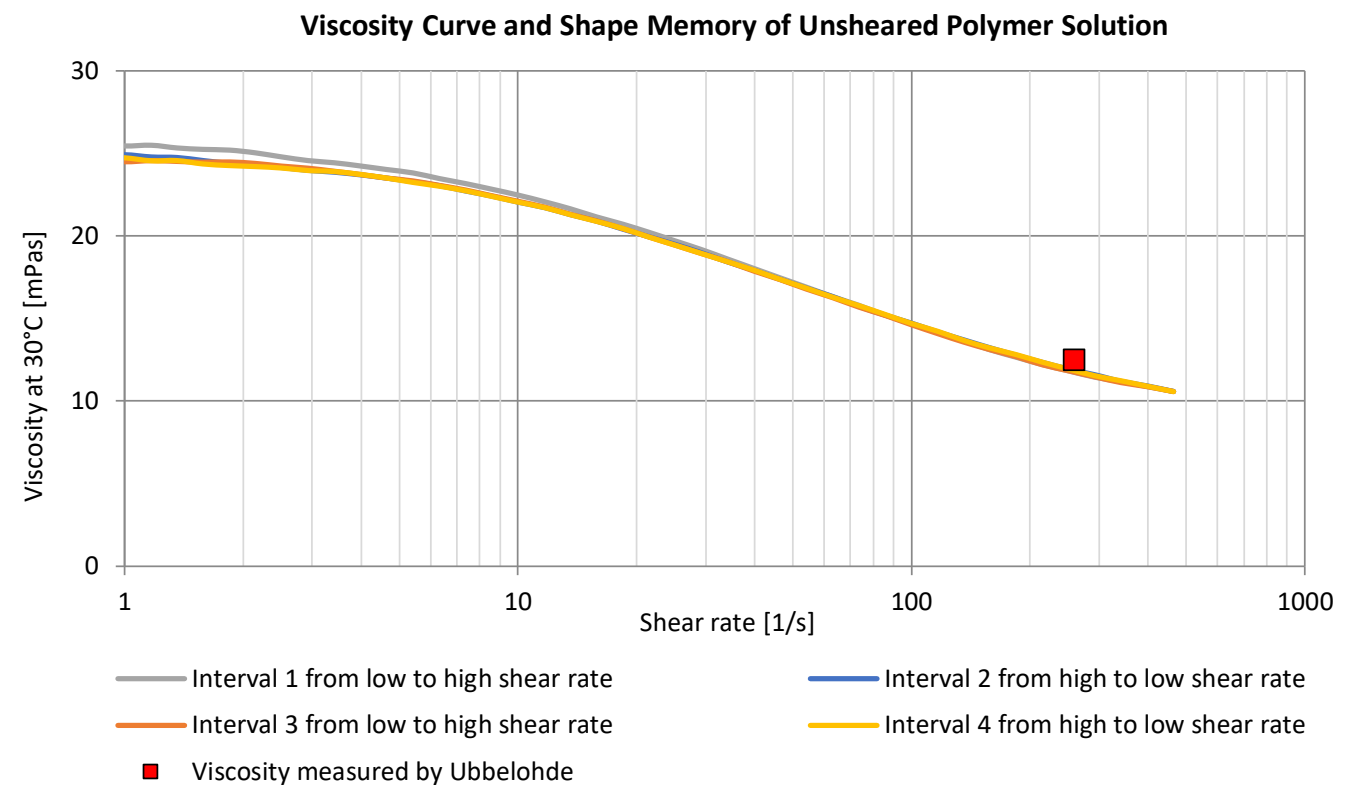

Figure 4-2: Viscosity stability at changing shear rate of unsheared polymer solution, running the solution from low to high, high to low, low to high, high to low shear rate measured with the Anton Paar rheometer, and viscosity measured with the Ubbelohde viscometer

\subsubsection{Polymer Viscoelasticity}

Because the shearing process during conditioning introduces deformation energy to the polymers, viscoelasticity measurements are exerted in order to find out to what extent the polymer is deformable without chain rupture. By measuring the complex shear modulus, $G^{*}$, of the polymer solution in oscillation at a shear stress range from 0.01 to $10 \mathrm{~Pa}$, the viscoelastic properties of the unsheared polymer solution is investigated. The viscoelastic behavior of the polymer solution during the amplitude sweep is shown in Figure 4-3. The linear viscoelastic range reaches until around 0.1 Pa before pseudoelastic behaviors are observed. As $G^{\prime \prime}$ is higher than $G^{\prime}$ in the linear viscoelastic range, the unsheared polymer solution behaves as an ideally viscous fluid having a liquid-like structure. This means that the polymer does not have a high ability to store deformation energy, and it shows with chain rupture during conditioning.

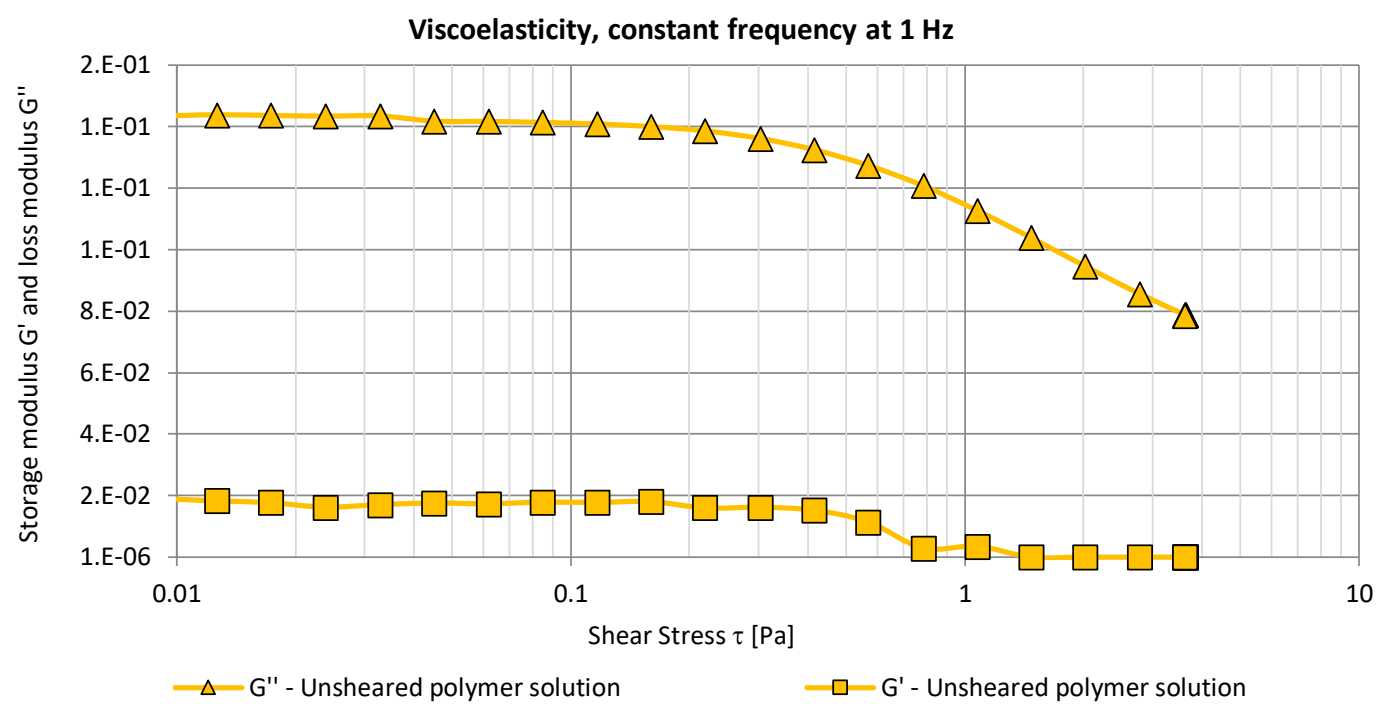

Figure 4-3: Viscoelasticity measurements of differently sheared polymer solutions at constant frequency 


\subsection{Rheology Fluid Characterization after Pre-conditioning}

This section describes the impact of shearing on rheological parameters and compares it to the unsheared polymer solution. The analysis includes screen factor, viscosity, critical polymer concentration, viscoelasticity, $\mathrm{pH}, \mathrm{molecular}$ weight and microscopic measurements of differently sheared polymer solutions.

\subsubsection{Device-dependent Rheological Response to Pre-conditioning}

The viscosity and the screen factor change after shearing, based on the initial values of the unsheared polymer solution, are examined for different shearing intensities using the disperser, turrax, and capillaries. This enables the establishment on how rheological parameters depend upon shearing intensity and of the corresponding correlations.

\section{a) Disperser-sheared Polymer Solution}

The polymer solution was sheared at 500, 750, 1000, 1250, 1500 and $1750 \mathrm{rpm}$ for both 30 and 60 min. Afterwards, the viscosity of the solution was measured by Ubbelohde viscometer, which is shown together with the determined mechanical degradation in Figure 4-4a. The screen factor and screen factor loss are shown in Figure 4-4b. At a rotational speed of $0 \mathrm{rpm}$, the values of the unsheared polymer solution are marked. It can be inferred that the rotational time does not have a significant impact on the rheological parameters. Furthermore, the viscosity is not as significantly affected by shearing as the screen factor is. While the viscosity drops by $4.6 \%$ after stirring for $30 \mathrm{~min}$ at $1750 \mathrm{rpm}$, the screen factor is reduced by $36 \%$. This indicates that the disperser highly disentangles the polymers, without causing a significant rupturing of the chain.
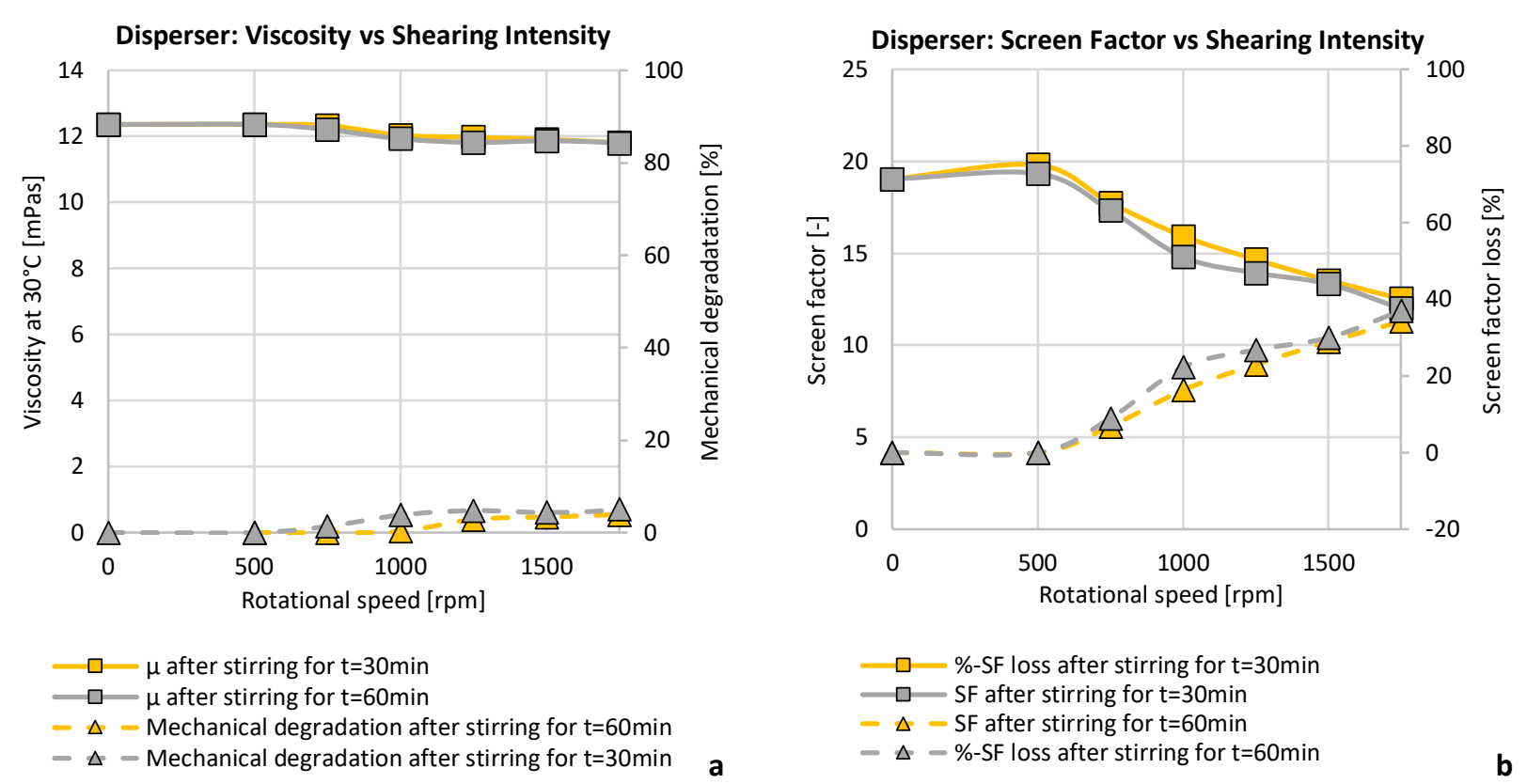

Figure 4-4: Viscosity measured by Ubbelohde and resulting mechanical degradation from the Ubbelohde [a] and screen factor and screen factor loss [b] from experiment series with disperser

\section{b) Turrax-sheared Polymer Solution}

The polymer solution was sheared with the turrax at 5,000, 10,000, and 20,000 rpm for 1, 3.25, 5.5, 7.75, and 10 min. The resulting rheological parameters after shearing with the turrax are shown in Figure 4-5, in which Figure 4-5a shows the Ubbelohde viscosity and mechanical degradation, and Figure 4-5b shows the screen factor and the screen factor loss. At $0 \mathrm{~min}$, the values of the unsheared polymer solutions are marked. As compared to the conditioning with the disperser, 
the turrax causes higher changes in viscosity and screen factor, reducing the viscosity by $28 \%$ and the screen factor by $71 \%$ when stirred for $10 \mathrm{~min}$ at $20,000 \mathrm{rpm}$.
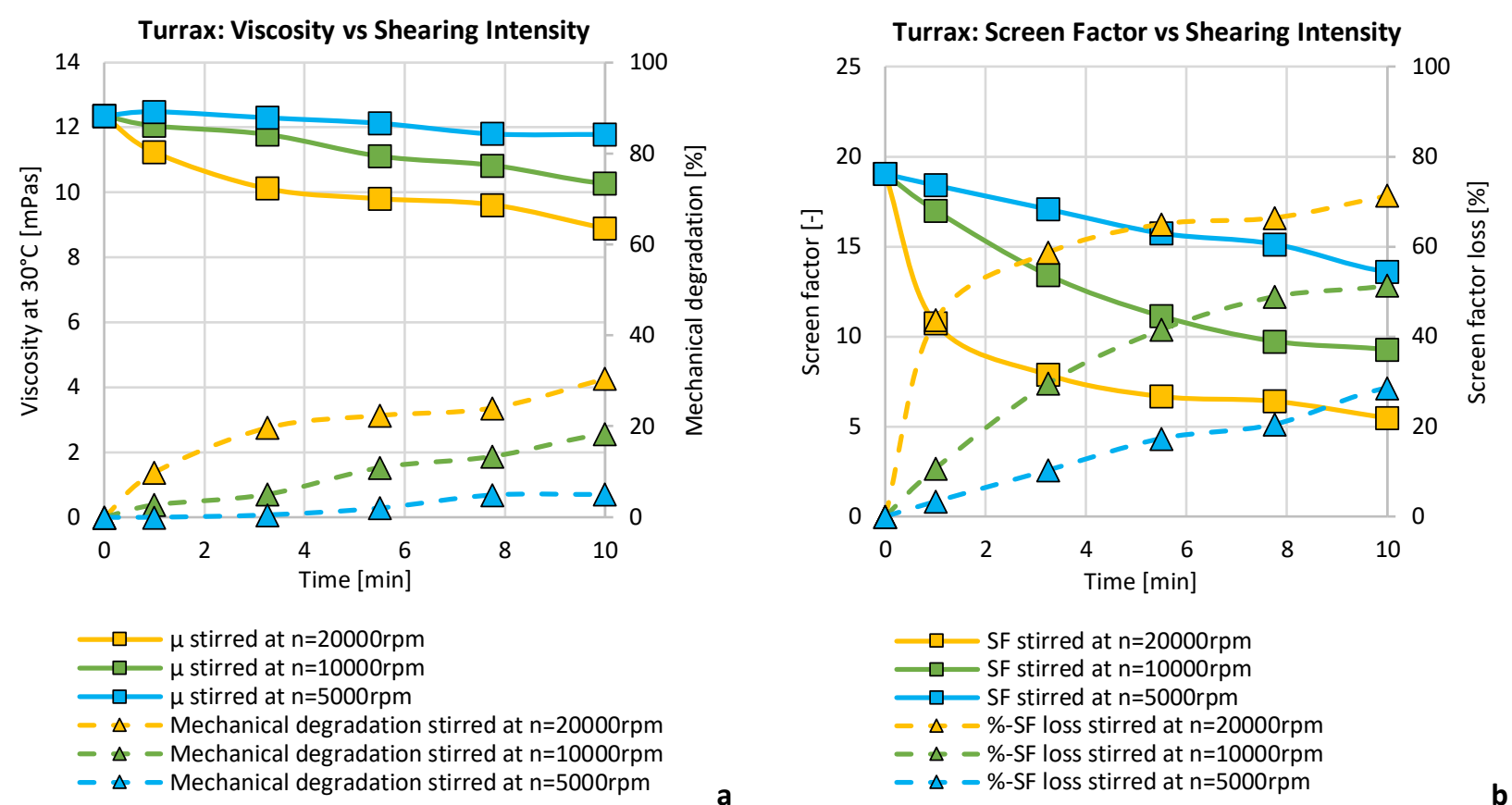

Figure 4-5: Viscosity measured by Ubbelohde and resulting mechanical degradation from Ubbelohde [a] and screen factor and screen factor loss [b] from experiment series with turrax

\section{c) Capillary-sheared Polymer Solution}

The unsheared polymer solution was sheared by forcing the solution into a capillary tube with an inner diameter of $1 \mathrm{~mm}$. The applied pressures are 1, 2, 3, 4, 5, 10, and 20 bar. The capillary lengths used are 3, 10, and $20 \mathrm{~cm}$. Figure 4-6 presents the results, whereby Figure 4-6a shows the Ubbelohde viscosity and the mechanical degradation, and Figure 4-6b shows the screen factor and the screen factor loss. It is observed that the mechanical degradation is higher for shorter capillaries due to the loss of pressure over the length, which increases with the length of the capillary. This means that the pressure at the entrance of the capillary is higher for shorter capillaries. It shows that mechanical degradation occurs at the entrance of the capillary, where turbulent flow is expected, while only little mechanical degradation occurs within the capillary. The highest measured viscosity loss is $14 \%$, which happens when a pressure of 20 bar is applied and a capillary with the length of $3 \mathrm{~cm}$ is used. The corresponding screen factor loss is $52 \%$. 

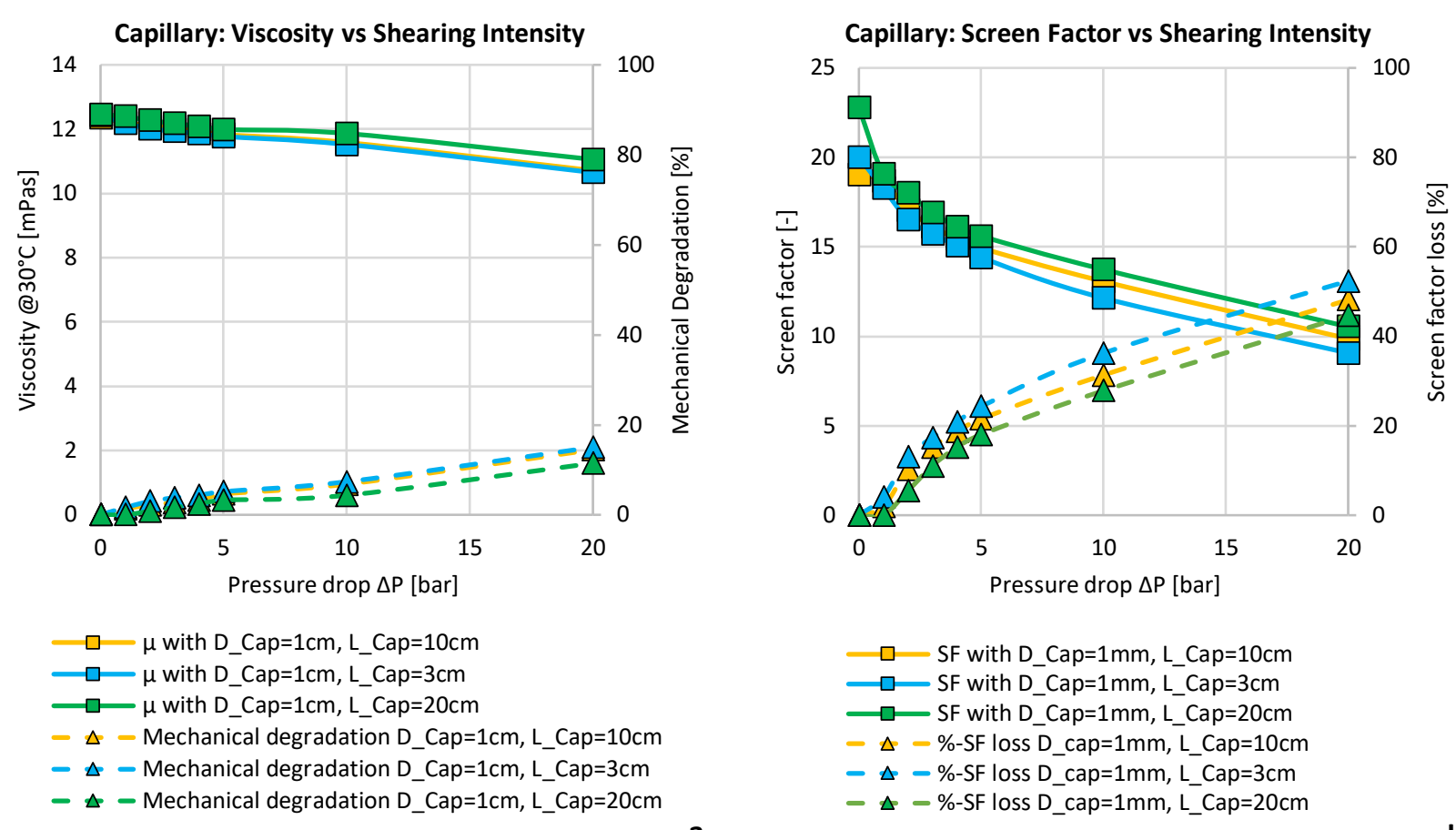

Figure 4-6: Viscosity measured by Ubbelohde and resulting mechanical degradation from Ubbelohde [a] and screen factor and screen factor loss [b] from experiment series with capillary

\section{d) Correlations between Rheology of Different Shear Origins}

Correlations between the rheology of the polymer solution conditioned by three different shearing devices are now investigated. The relation between the viscosity and screen factor of differently sheared polymer solutions is examined. This is done by plotting all the results of the viscosity and screen factor of the sheared polymer solutions by the disperser, turrax and capillaries shown in the above sections a), b) and c) against each other. Interestingly, this gives a perfectly matching trend as shown in Figure 4-7. This means that no matter which source of degradation or intensity is chosen, the viscosity and screen factor respond in the same way, providing a rheological master curve for the polymer system PS- $A$, which is shear-origin independent.

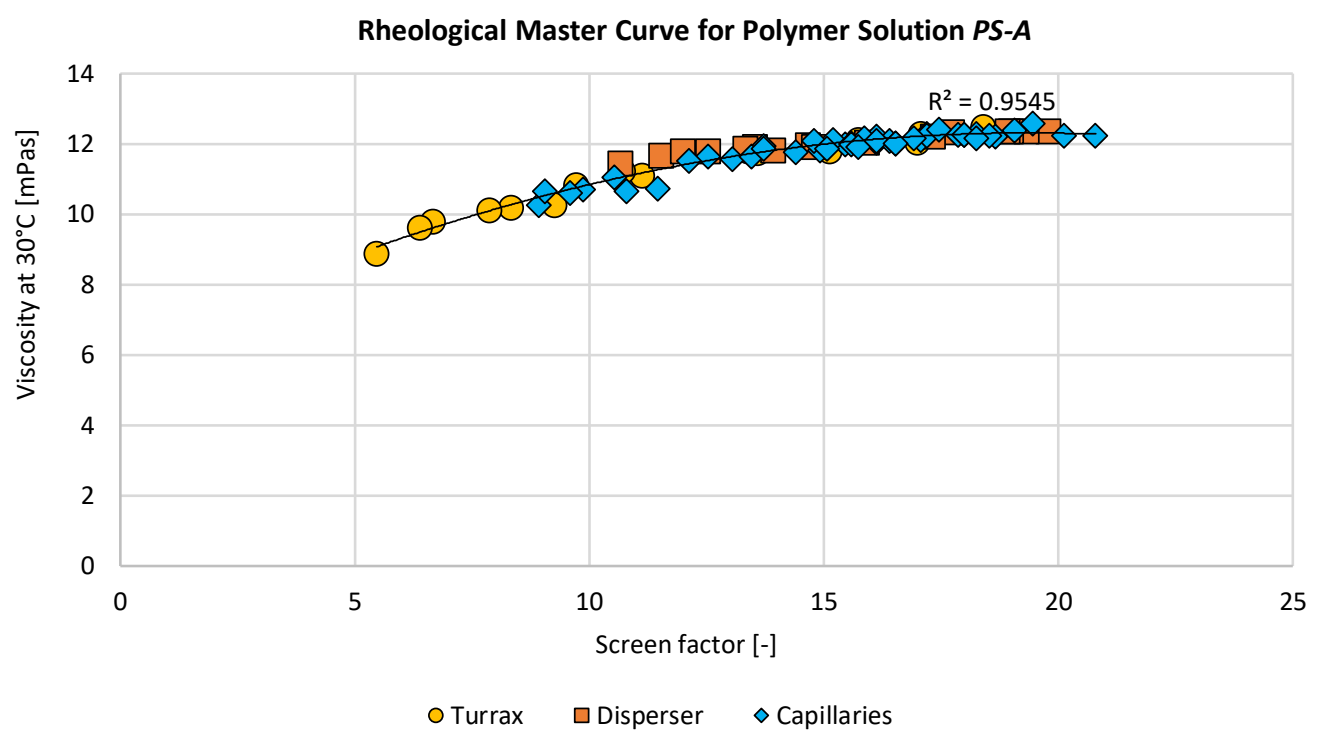

Figure 4-7: Rheological master curve between viscosity measured by Ubbelohde and screen factor after shearing polymer solution with different shearing devices 
The volume-specific energy input, $E_{v}$, of the polymer solution can be determined for all shearing devices. This allows a direct comparison of the rheological response with the different shearing devices. To determine $E_{v}$, Reynolds number for agitators, $R_{e}$, and the power number, $N_{p}$, need to be determined according to Equation 2-28 and 2-29 respectively. $R_{e}$ can be determined for each solution, taking density, rotational speed, diameter of the mixer, and the final viscosity of the solution as input. $N_{p}$ is then determined through the turbine power correlations, which are empirical geometry dependent correlation, based on Rushton, Costrich \& Everitt's work (1950), and it has been adopted ever since in the agitator research (Bates, Fondy, \& Corpstein, 1963; Hershey \& Brodkey, 1988).

The existing turbine-power correlation for disperser and disk stirrer is shown in Figure 4-8. The turrax geometry is similar to the disk stirrer, yet it provides a higher energy input due to the rotor and stator components. Thus, the curve has been shifted by matching the rheological data of the turrax-sheared polymer solutions to the disperser-sheared solutions (see Figure 4-8) in order to provide a realistic $N_{p}$.

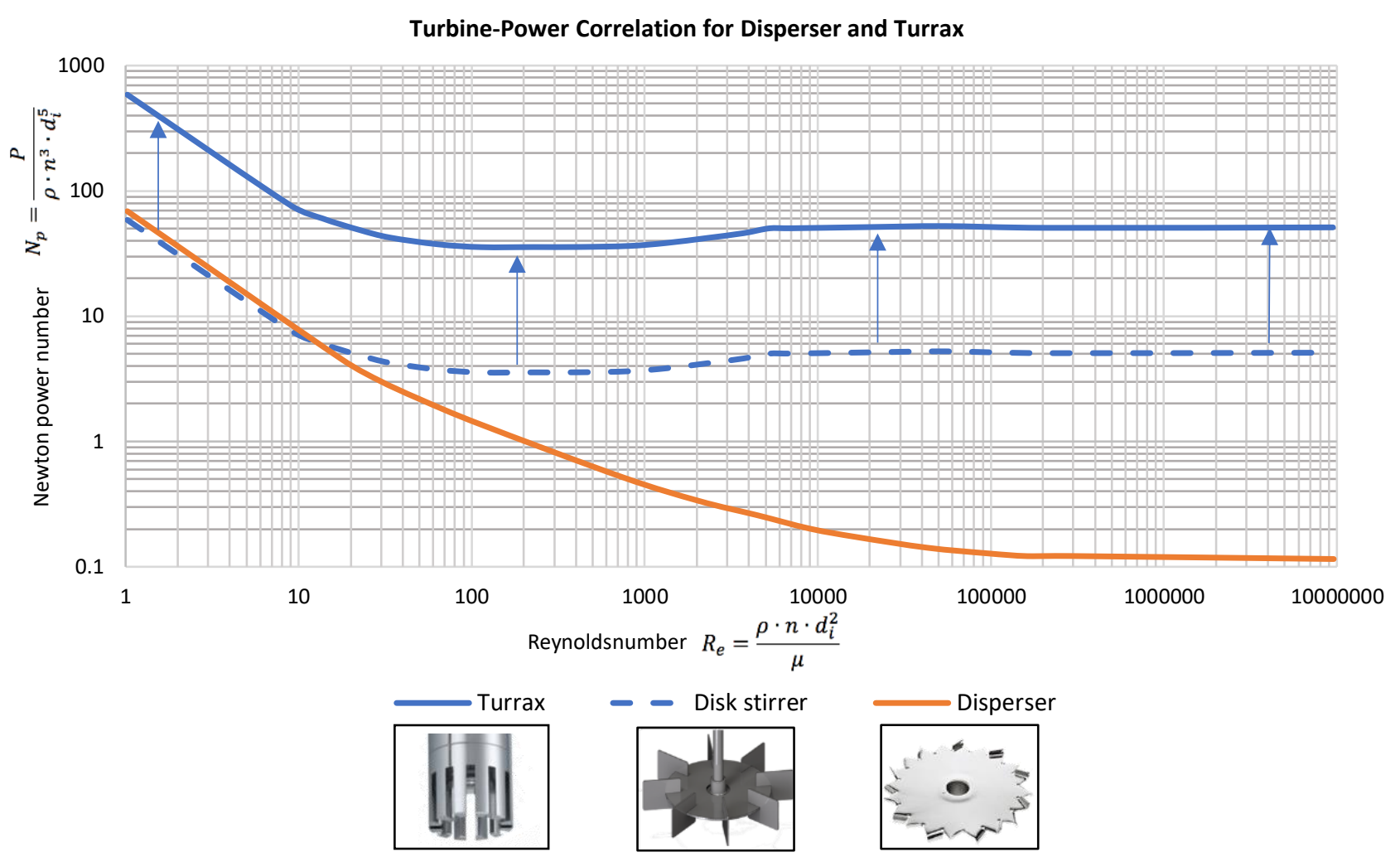

Figure 4-8: Turbine-power correlation for disperser and turrax adapted from Hershey \& Brodkey (1988), Bartes, Fondy \& Corpstein (1963) and Rushton, Costrich \& Everitt (1950)

The curves for both disperser and turrax are shown in Figure 4-9. It can be seen that, also here, another unique master curve emerges, which allows for the determination of rheological parameters, such as the viscosity or the screen factor.

As shown in Figure 4-9b, both viscosity and screen factor approach asymptotic values. This means that with an increase in the shearing intensity, there is a reduction in the loss of viscosity and screen factor. This can be explained by the destruction of the high-end of the MWD. 

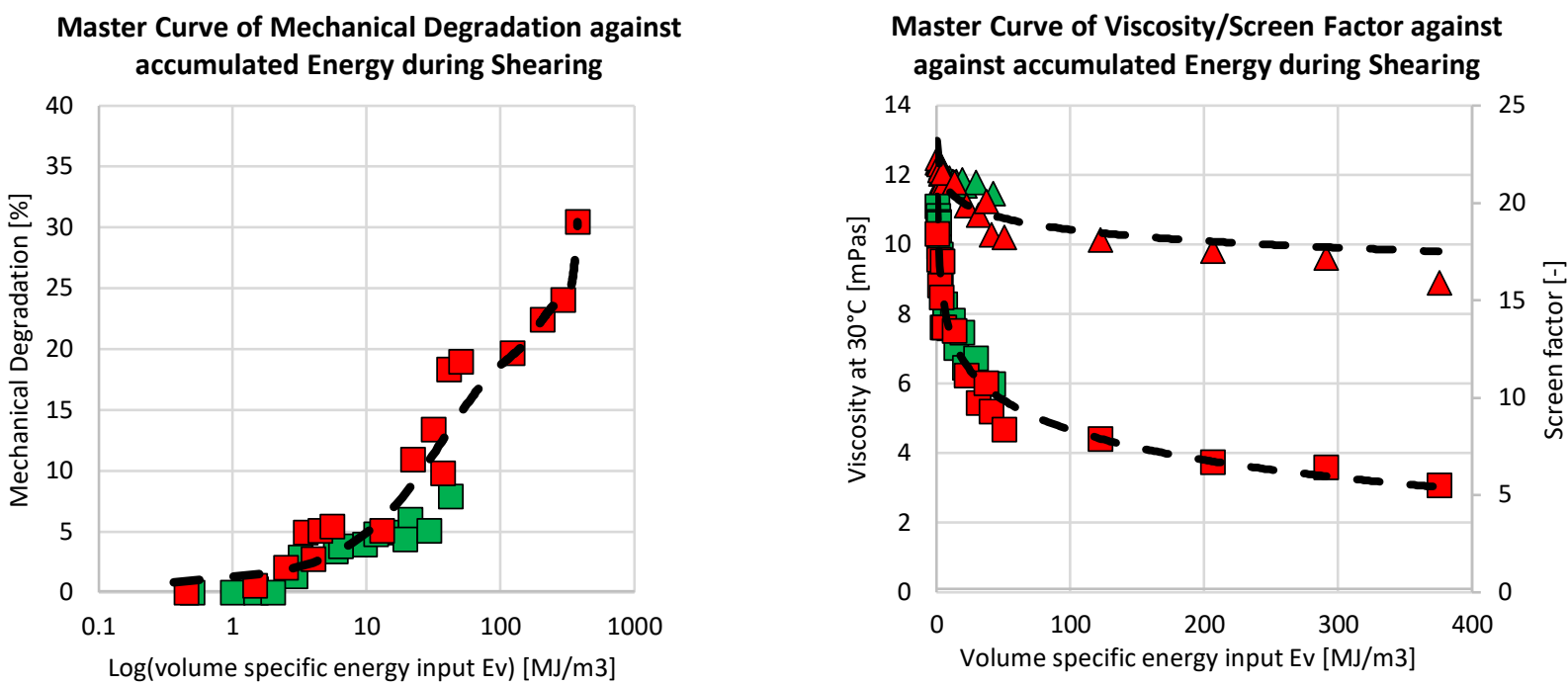

$\square$ Disperser $\quad \square$ Turrax
$\Delta$ Viscosity with Disperser
$\Delta$ Viscosity with Turrax
$\square$ Screen factor with Disperser
$\square$ Screen factor with Turrax

Figure 4-9: Master curve of mechanical degradation against Ev [a], and viscosity measured with Ubbelohde and screen factor against Ev [b] for disperser and turrax

The strong trend between the viscosity and screen factor at different shearing intensities, as shown in Figure 4-10, indicates that the flow in contractions through capillary tubes also applies to the master curve. By using the regression function of the screen factor versus $E_{v}$, resulting from the master curve of turrax-sheared and disperser-sheared polymer solutions, the viscosity and screen factor with respect to $E_{v}$ can be added to the master curve as shown in Figure 4-10.

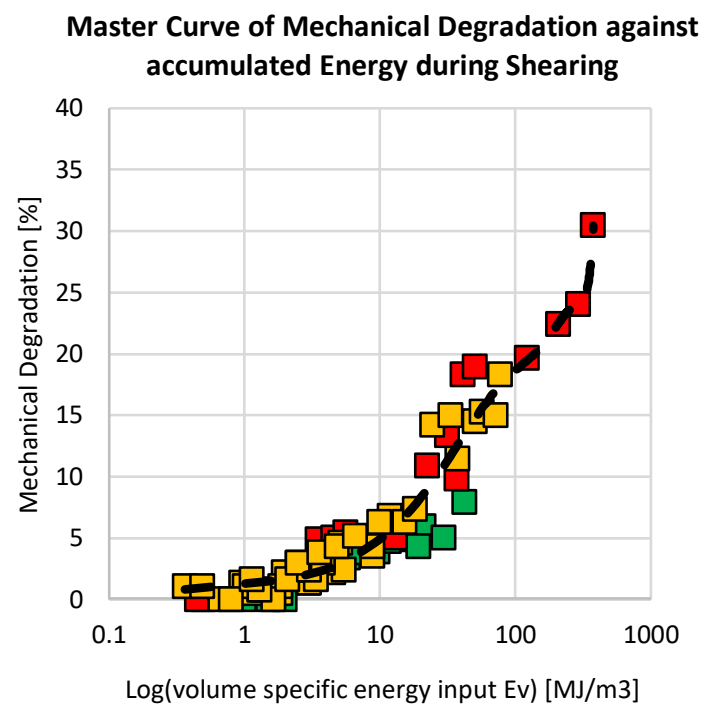

$\square$ Disperser $\quad \square$ Turrax $\square$ Capillaries

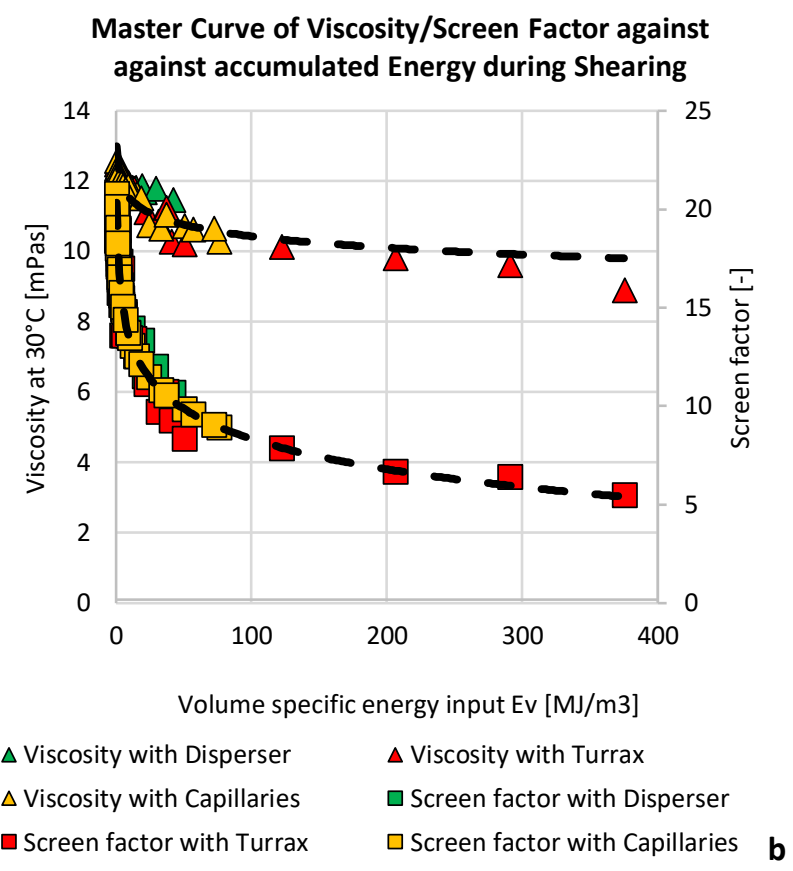

Figure 4-10: Master curve of mechanical degradation against Ev [a], and viscosity measured with Ubbelohde and screen factor against Ev [b] for disperser, turrax and capillaries 


\subsubsection{Critical Polymer Concentration}

To investigate on the effect of shearing on the polymer concentration, the polymer solution is sheared at different polymer concentrations. The results are shown in Figure 4-11. As expected, the viscosity of the polymer solutions reduces in all concentration regimes due to the increased disentanglement of polymer chains and the mechanical degradation of the high-end of the MWD. Except for one outlier in the $C^{*}$ intersection, $C^{*}$ and $C^{C}$ grow with increasing shearing intensity. Nevertheless, the polymer solution remains at the threshold of the concentrated regime for all sheared polymer solutions.

Ubbelohde Viscosity vs Polymer Concentration - Differently Pre-sheared Polymer Solutions

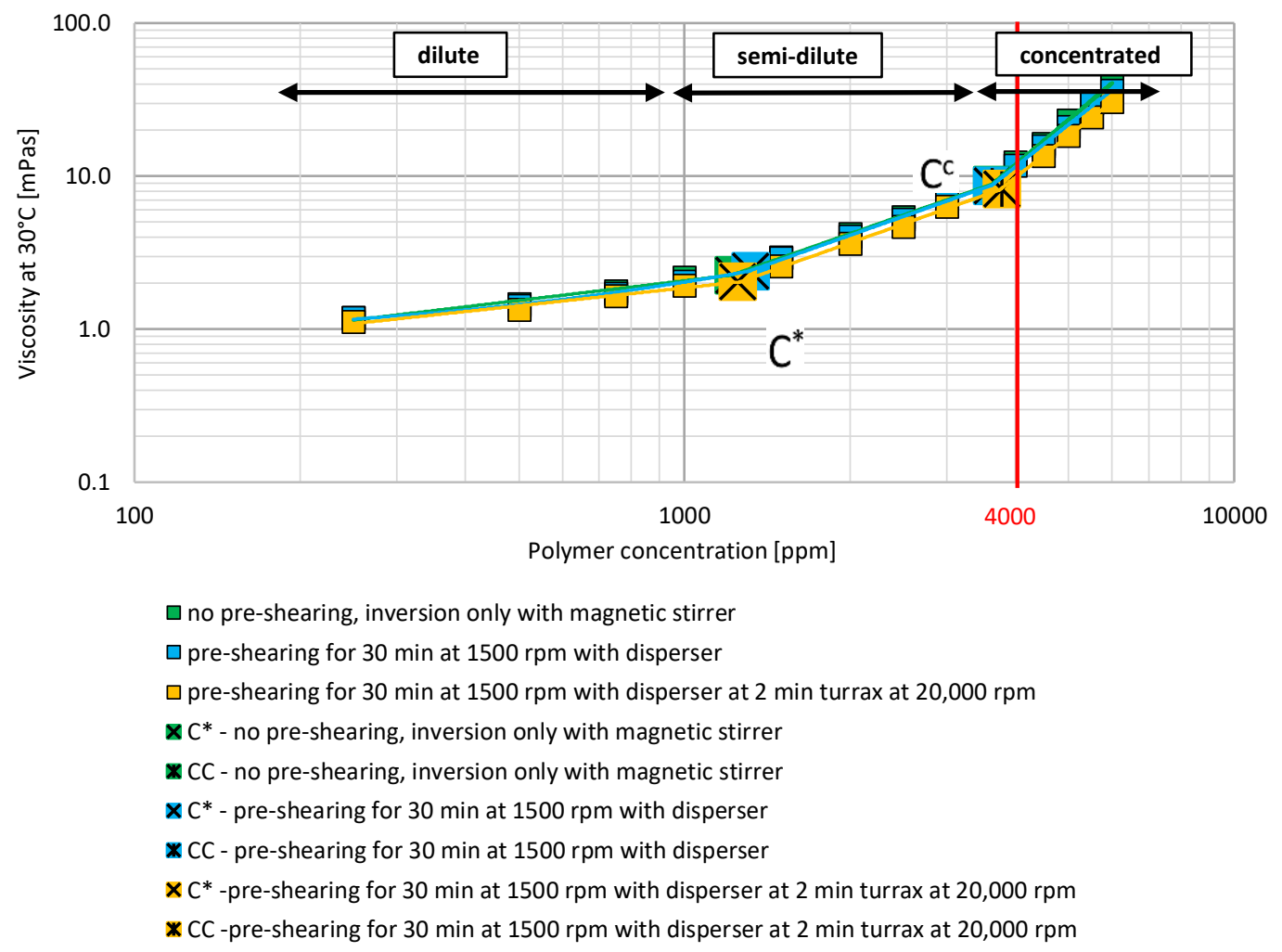

Figure 4-11: Viscosity from Ubbelohde vs polymer concentration - differently pre-sheared polymer solutions with 2,400 ppm inverter surfactant $I S-A$

The investigation of the test series with a rheometer at a higher shear rate of 250 rps shows that the distinction between semi-dilute and concentrated regime becomes less visible. Still, two separate trend lines can still be determined allowing for the location of the critical concentration $C^{C}$ (see Figure 4-12). The same location of $C^{C}$ can be determined for shear rates up to $1,000 \mathrm{rps}$. This means that the investigated polymer system at 4,000 ppm remains in concentrated regime, also with varying shear rates. Further, pre-shearing of the polymer solution at different shearing intensities also does not destroy the viscosifying effect and the solution remains in concentrated regime. 


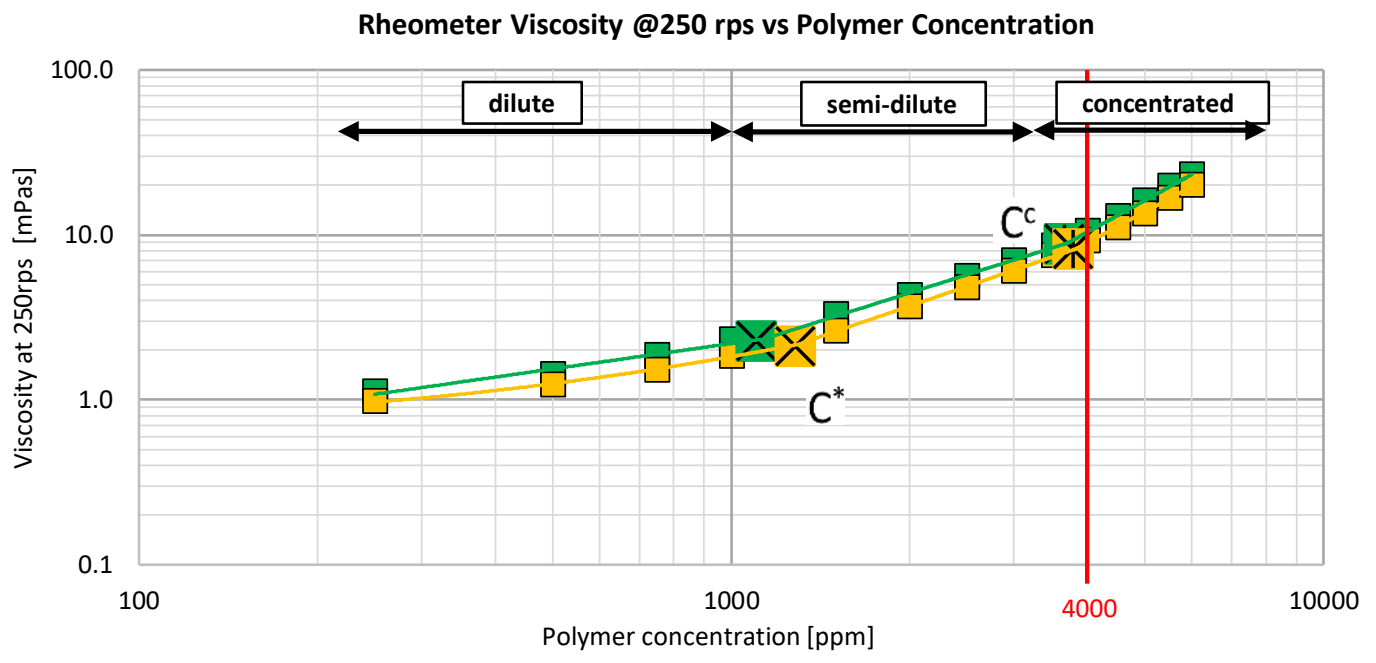

$\square$ at 250rps - no pre-shearing, inversion only with magnetic stirrer

$\square$ at $250 \mathrm{rps}$ - after $30 \mathrm{~min}$ at $1500 \mathrm{rpm}$ with disperser at $2 \mathrm{~min}$ turrax at 20,000 rpm

$\mathbf{x} C^{*}$ - no pre-shearing, inversion only with magnetic stirrer

* CC - no pre-shearing, inversion only with magnetic stirrer

X C* -pre-shearing for $30 \mathrm{~min}$ at $1500 \mathrm{rpm}$ with disperser at $2 \mathrm{~min}$ turrax at 20,000 rpm

× CC -pre-shearing for $30 \mathrm{~min}$ at $1500 \mathrm{rpm}$ with disperser at $2 \mathrm{~min}$ turrax at 20,000 rpm

Figure 4-12: Viscosity at 250 rps from R/S+ Rheometer vs polymer concentration measured with Brookfield R/S+ Rheometerdifferently sheared polymer solutions with $2,400 \mathrm{ppm}$ inverter surfactant IS-A

\subsubsection{Polymer Viscosity}

The viscosity curves for differently shearing intensity have been determined against shear rate by the Anton Paar rheometer, and the results are shown in Figure 4-13. By increasing the pre-shearing intensity, the zero-rate viscosity decreases, while the critical shear rate (i.e. the transition point from Newtonian to shear thinning behavior) increases (marked in Figure 4-13 determined by matching curves to Carreau model). Consequently, the relaxation time, which is the inverse of the critical shear rate, decreases, the higher the pre-shearing intensity. This means that the polymers require less time to relax, i.e. to contract from extended state, the more it is pre-sheared, leading to a longer Newtonian lasting plateau. 


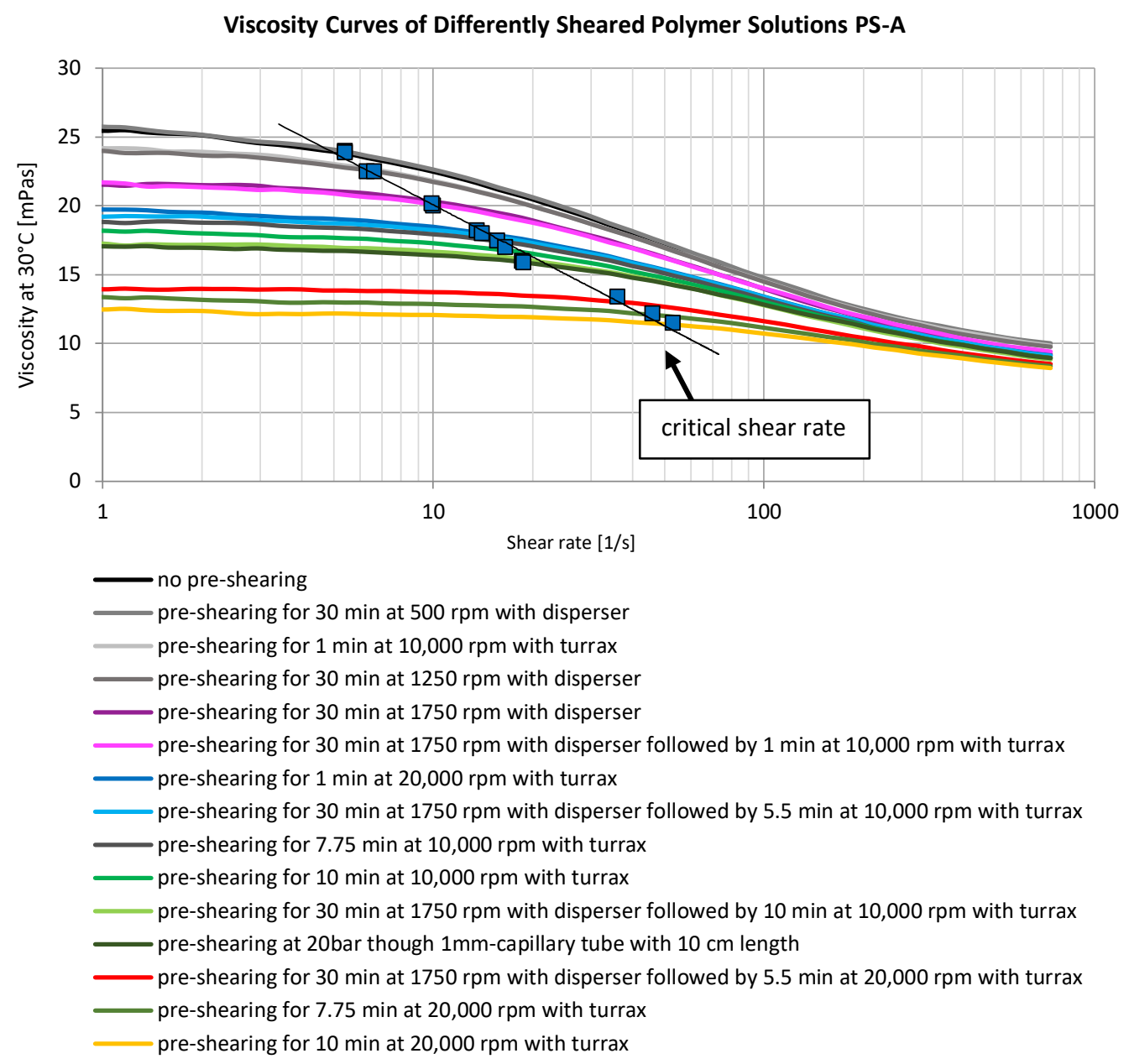

Figure 4-13: Viscosity curves with increasing shearing intensity measured with Anton Paar M 302

\subsubsection{Polymer Viscoelasticity}

The complex shear modulus, $G^{*}$, is determined for differently sheared polymer solutions. As can be seen in Figure 4-14, both $G^{\prime}$ and $G^{\prime \prime}$ reduce with the increase in shearing intensity of the polymer solution. At the same time, the capacity of the fluid to maintain its viscosity increases with the increasing shearing intensity, which can be observed in the duration of the plateau behavior of the loss modulus, $G^{\prime \prime}$. Therefore, the drop of viscosity loss occurs at higher shear stress, i.e. with the increase in the shearing intensity of the polymer solution. After the $G^{\prime \prime}$ drop, a delayed reduction in $G^{\prime}$ occurs. Likewise as the unsheared solution, the sheared polymer solution also behaves as an ideally viscous fluid with a liquidlike structure for sheared polymer solutions. 


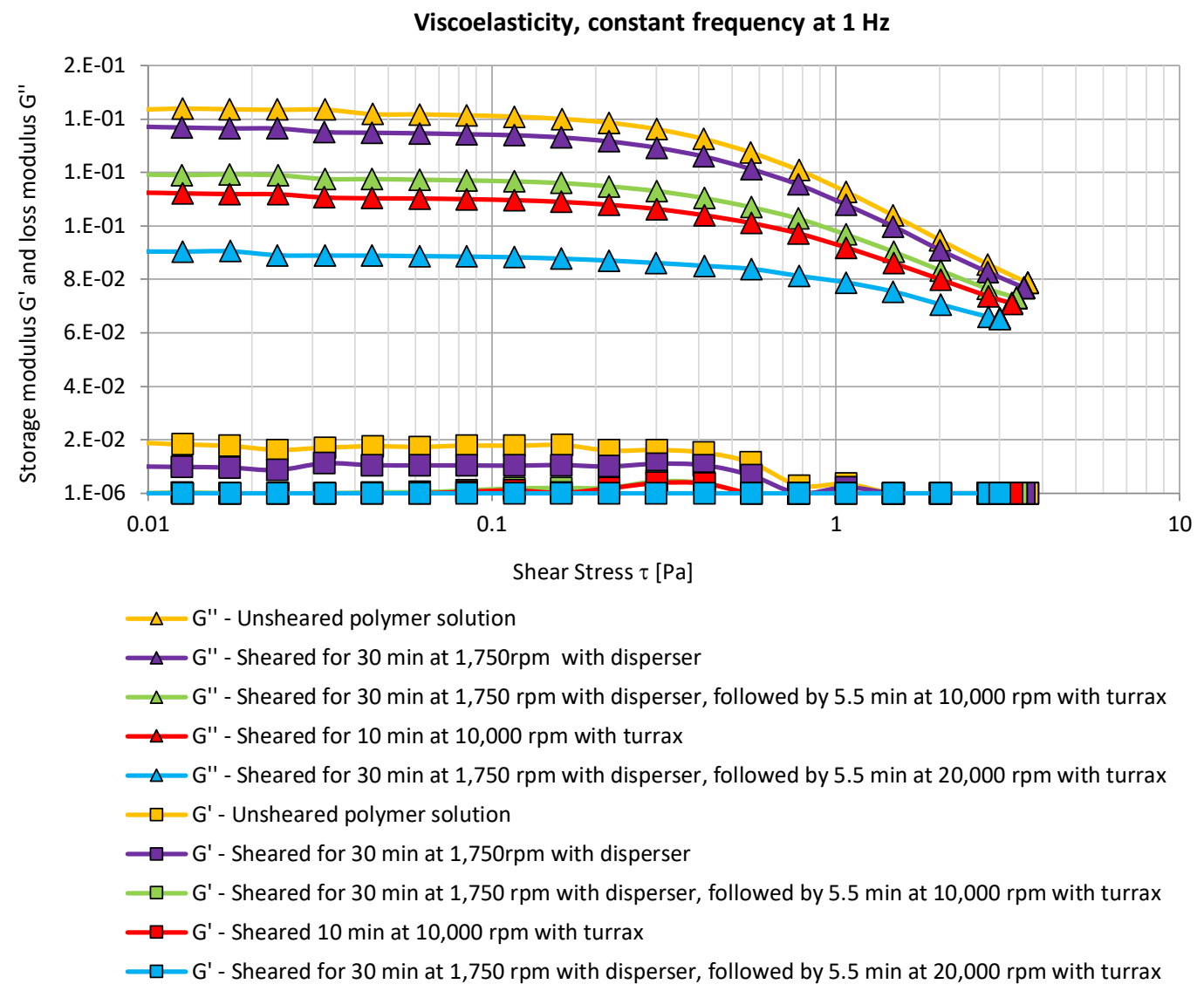

Figure 4-14: Viscoelasticity measurements of differently sheared polymer solutions at constant frequency measured with Anton Paar M 302

\subsection{5 pH Measurement}

The $\mathrm{pH}$ was determined for all polymer solutions. Even though, the different sheared polymer solutions fall under the same master curves as explained in section 4.2.1, a slight increase in $\mathrm{pH}$ between the differently sheared polymer solutions depending on the shear origin can be recognized (see Figure 4-15).

There are several reasons that could explain such a shear-origin dependent increase in $\mathrm{pH}$. The most plausible reason for the change in the $\mathrm{pH}$ could be that the mechanical degradation of inverter surfactant $I S-A$, which is highly shear sensitive, occurs. Shearing with a disperser causes a higher turbulence, as compared to the capillaries and turrax, leading to a high foam formation. However, the hydrolysis of $I S-A$ could happen faster due to the introduction of the air, which causes the formation of foam. The increasing foam formation, increases the degradation of $I S-A$, which, in turns, causes the $\mathrm{pH}$ to move to alkaline milieu. This, however, does not impact the measured rheology including viscosity and screen factor. 


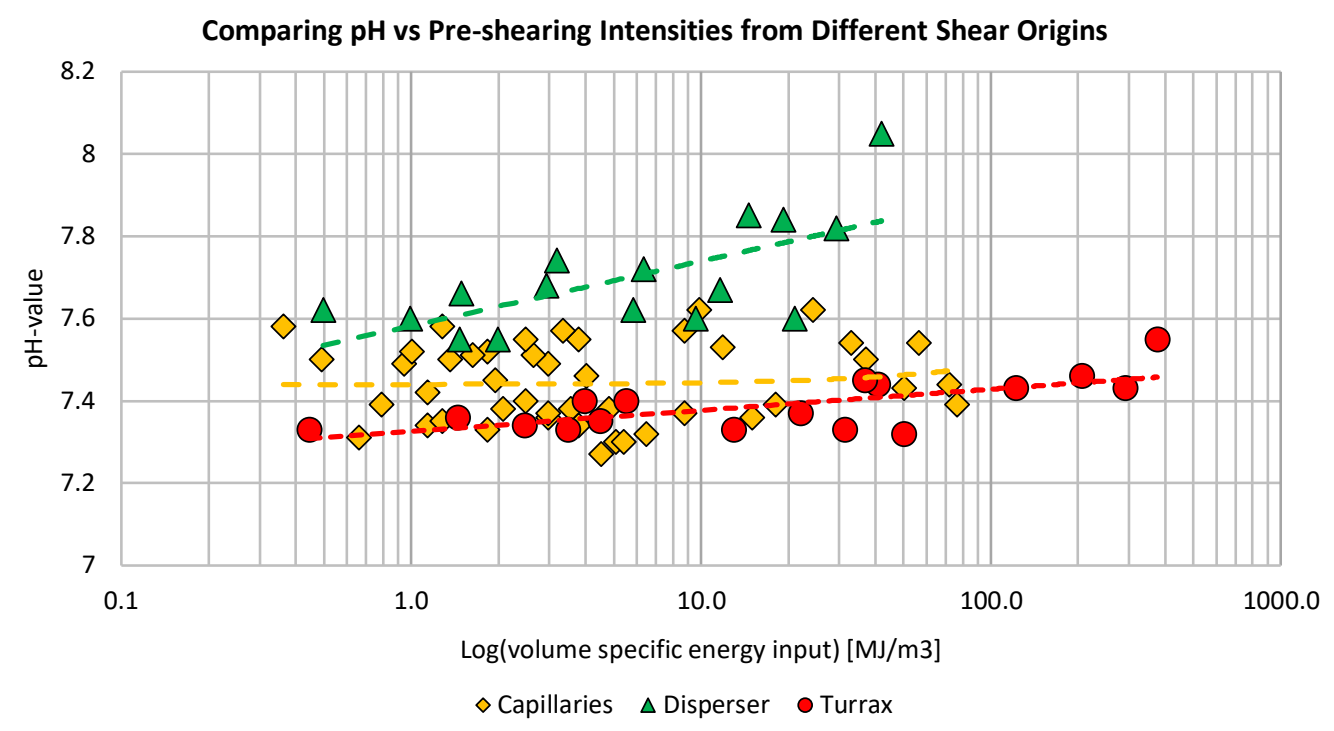

Figure 4-15: pH after pre-shearing with different shearing intensities and devices

\subsubsection{Molecular Weight}

The average molecular weight of differently sheared polymer solutions was determined using Equation 2-8 and it has been plotted relatively against $E_{v}$ imposed by shearing, as shown in Figure 4-16. As described above, the forced flow through capillary tubes nearly stays constant for 1 to 5 bar, which is represented by the first five data points shown in Figure 4-16. It, then, drops at higher pressures.

Shearing with the disperser for $30 \mathrm{~min}$ at $1750 \mathrm{rpm}$ reduces the average molecular weight from 1 to 0.84 . The molecular weight is significantly reduced by shearing with turrax, which also inputs a comparably higher energy input. The molecular weight of polymer solutions sheared with the turrax only for 1 and $3 \mathrm{~min}$ at 20,000 rpm is significantly higher at 0.86 and 0.84 respectively at the determined energy input than the molecular weight of the polymer solution sheared with the turrax for $7.75 \mathrm{~min}$ at $10,000 \mathrm{rpm}$ at a lower energy input, where the molecular weight drops to 0.72 . By shearing the polymer solution for $7.75 \mathrm{~min}$ at 20,000 rpm, the molecular weight drops to 0.67 . The increased molecular weight sheared at 1 and $3 \mathrm{~min}$ indicates that the residence time for the sheared volume was too short to shear the entire polymer solution. This left a few chains unsheared, resulting in a higher molecular weight. 


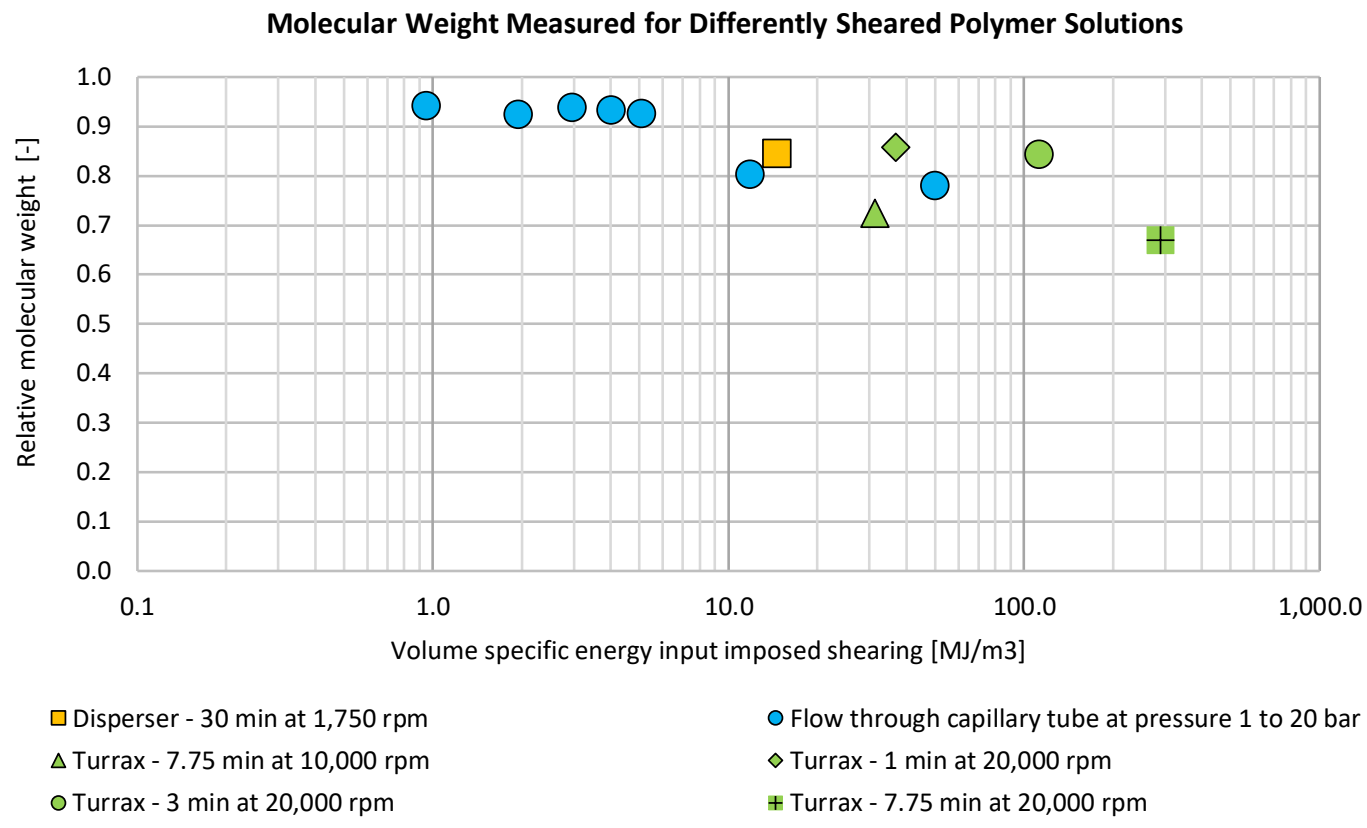

Figure 4-16: Molecular weight measured for differently sheared polymer solutions versus accumulated energy input during preshearing

\subsubsection{Viscosity of Conditioned Solution in Capillary Tubes}

For capillary tube experiment conducted at 1, 2, 3, 4, 5, 10, and 20 bar with three different capillary lengths of 3, 10, and $20 \mathrm{~mm}$, the shear rate and relative apparent viscosity was determined by using Equation 2-20 and Equation 2-23. The corresponding graph is represented in Figure 4-17. In the shear rate range of 6E03 to $8 \mathrm{E} 03$ rps, an increase in relative apparent viscosity can be observed for the shorter capillaries of 3 and $10 \mathrm{~mm}$ length, in which the intensity of the viscosity rise increases with the decrease in capillary length. This is due to the fact that the shorter the capillary, the higher the pressure at the entrance of the capillary. This causes more turbulence and, thereby, less relaxation time. The capillary having the longest length of $20 \mathrm{~mm}$ only shows shear thinning behavior, and no increase in the apparent viscosity. Interestingly, the relative apparent viscosity also of the shorter capillary lengths reduces again at higher pressure or shear rates, becoming aligned again with the apparent viscosity of the 20-mm capillary. This could indicate that there is an area where pressure-dependent shear thickening behavior occurs. But, when the pressure or shear rate gets too high, this behavior disappears again and returns to shear-thinning due to the mechanical degradation of polymer chains at higher pressures as shown in Figure 4-6b. Also, the study of Al Hashmi, et al. (2013) a similar behavior of shearing thinning, shear thickening followed by shear thinning could be observed. 
Relative Apparent Viscosity vs Shear Rate

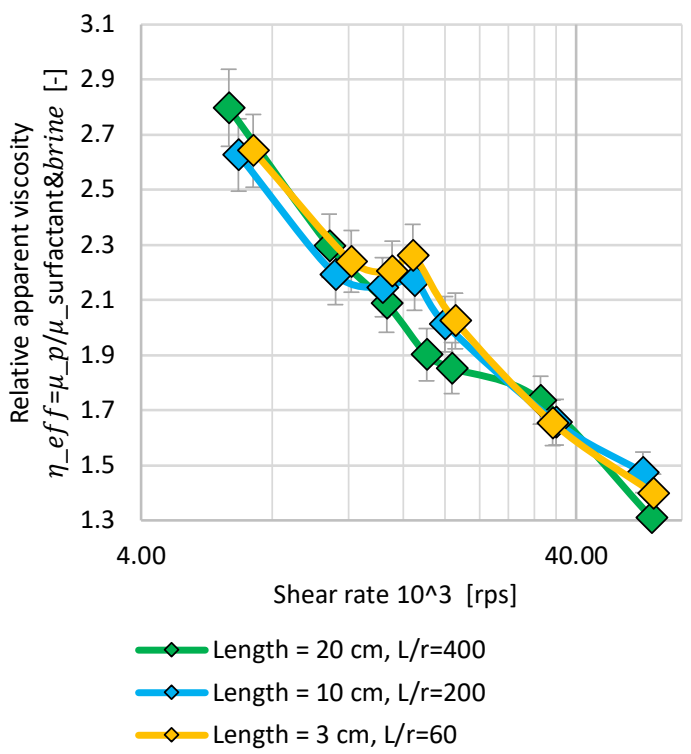

Relative Apparent Viscosity vs Pressure Drop

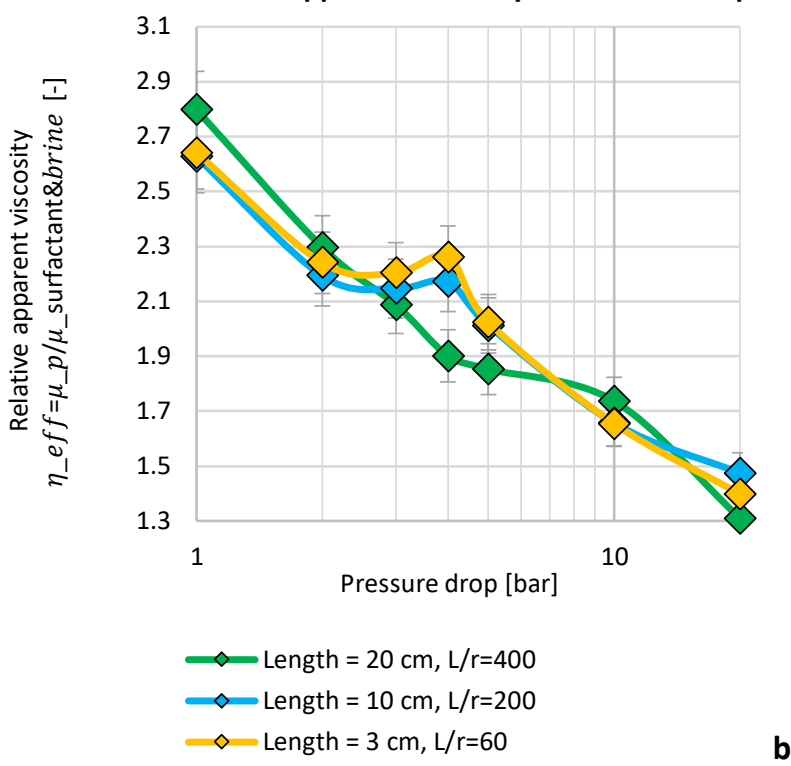

Figure 4-17: Relative apparent viscosity vs shear rate [a], relative apparent viscosity vs pressure drop [b]

The molecular weight for the capillary with the length of $10 \mathrm{~cm}$ was determined for fluids forced through the capillaries at different pressures by measuring the viscosity of the extracted polymer dissolved in distilled water at different concentrations. The resulting molecular weight derived from the Mark-Houwink equation and the measured viscosity at $30^{\circ} \mathrm{C}$ against the applied pressure drop in the capillary experiment are shown in Figure 4-18.

It can be observed that the molecular weight as well as the viscosity stay relatively constant till a pressure of 5 bar. After the pressure of 5 bar, the molecular weight drops by $16 \%$ at 10 bar. This indicated that there was none or very small changes in the length of the polymer chain, while a change in the screen factor can be observed, meaning that the polymer is disentangled, i.e. stretched until a pressure of 5 bar, without causing a rupture in the chain.

Also, the constant values of molecular weight and the measured viscosity until 5 bar could be another argument indicating that the observed increase in apparent viscosity is a temporary shear thickening behavior. Also here, the shear thinning behavior due to the mechanical degradation of the polymer is shown by the reduction in viscosity and molecular weight.

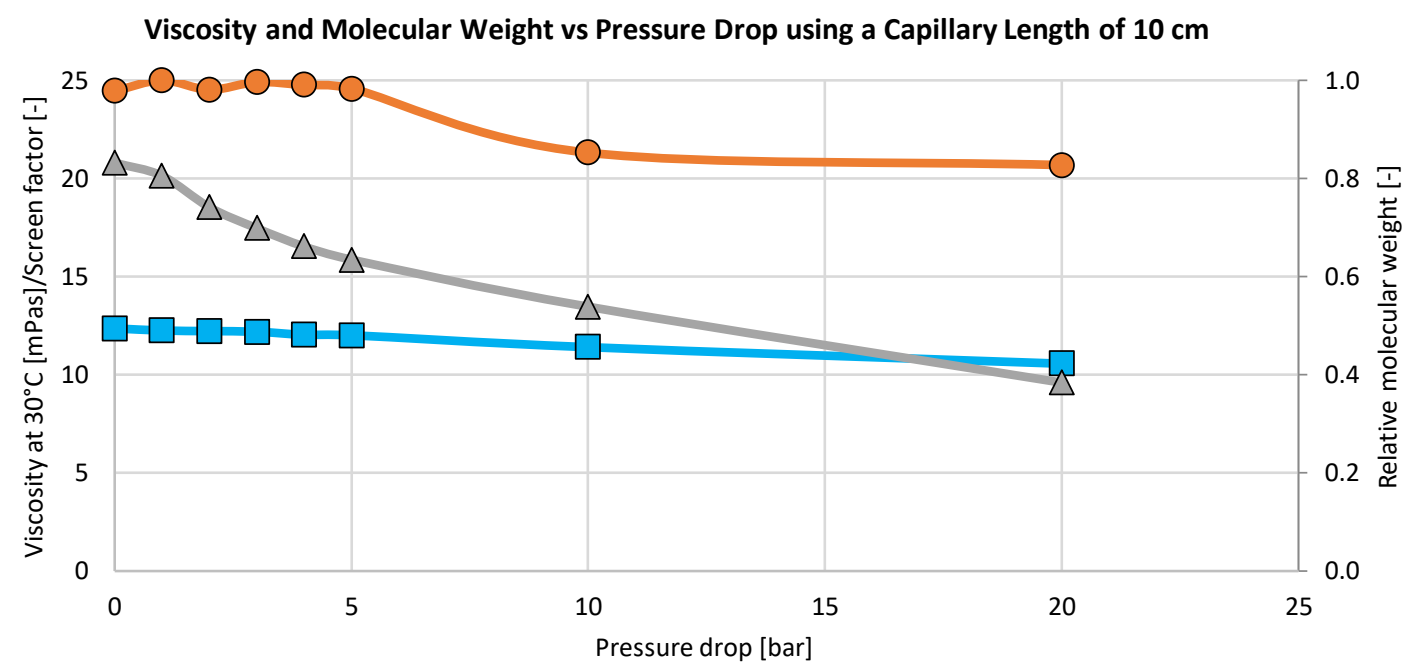

$$
\square \text { Ubbelohde Viscosity at } 30^{\circ} \mathrm{C} \quad \Delta \text { Screen factor } \quad \text { Molecular Weight }
$$

Figure 4-18: Viscosity measured with Ubbelohde and molecular weight vs pressure drop for capillary tube with 1 mm diameter and $10 \mathrm{~cm}$ length 


\subsubsection{Time-dependent Fluid Stability}

To examine whether the sheared polymers remain stable over several days and do not recombine, the solution were stored for several days at room temperature, and both viscosity and screen factor were measured over time. After three days, the milky-like color of the polymer solution clears up, and a layer of oil is formed on the surface of the solution due to the insolubility and the difference in density. After 13 days, approximately $40 \%$ of the oil droplets still remain dispersed in the solution, while the rest rose to the surface. The polymer solution at 0 and 13 days is shown in Figure $4-19 a$ and $b$. As shown in Figure 4-19c, the viscosity and the screen factor of polymer solution remain stable over the measured time period, which means that short-time storage and a reduced oil concentration do not impact the polymer.
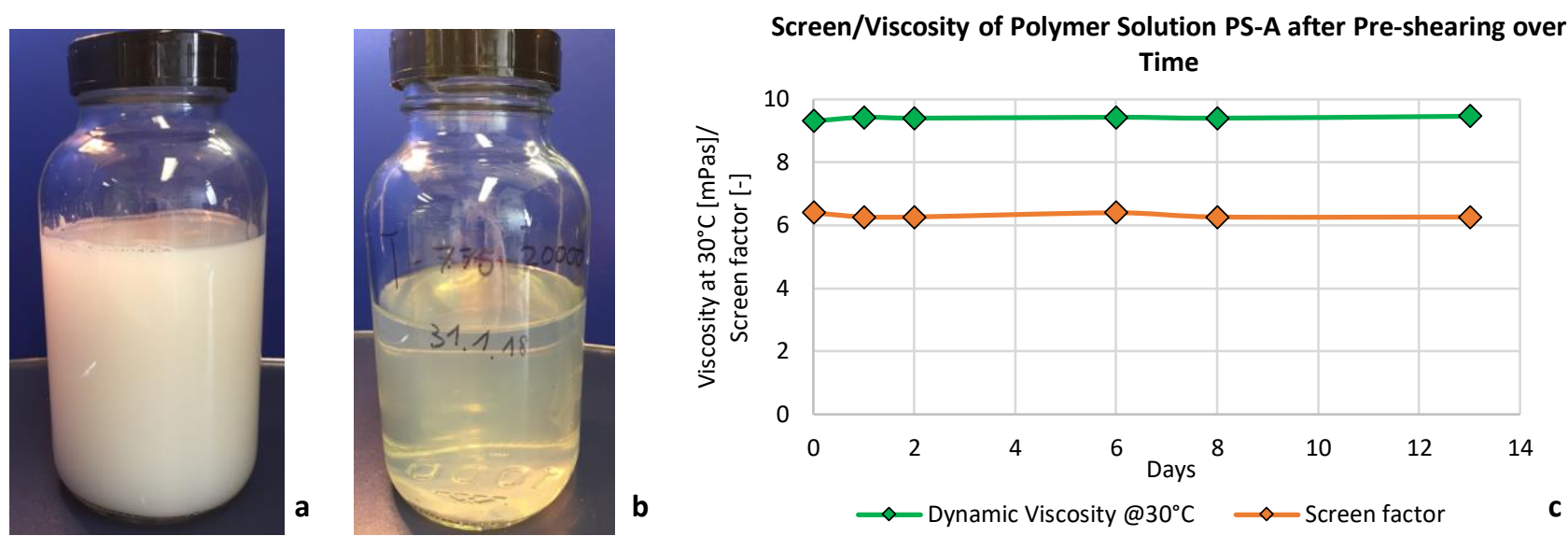

Figure 4-19: Pre-sheared polymer solution at day 0 [a] and day 13 [b] and Polymer stability of sheared polymer solution over time (viscosity from Ubbelohde) [c]

\subsubsection{Microscopic Images}

In order to have an understanding of the size of the oil droplets at different shearing intensities, the oil phase in the polymer solution was colored and viewed under the microscope. The microscopic images are shown in Figure 4-20. The oil droplets have been colored green. No visible difference can be observed in the size of the oil droplet size between the unsheared and the sheared polymer solution. This indicates that the oil should not affect the pre-sheared polymer solutions in a different way (see Figure 4-20b).
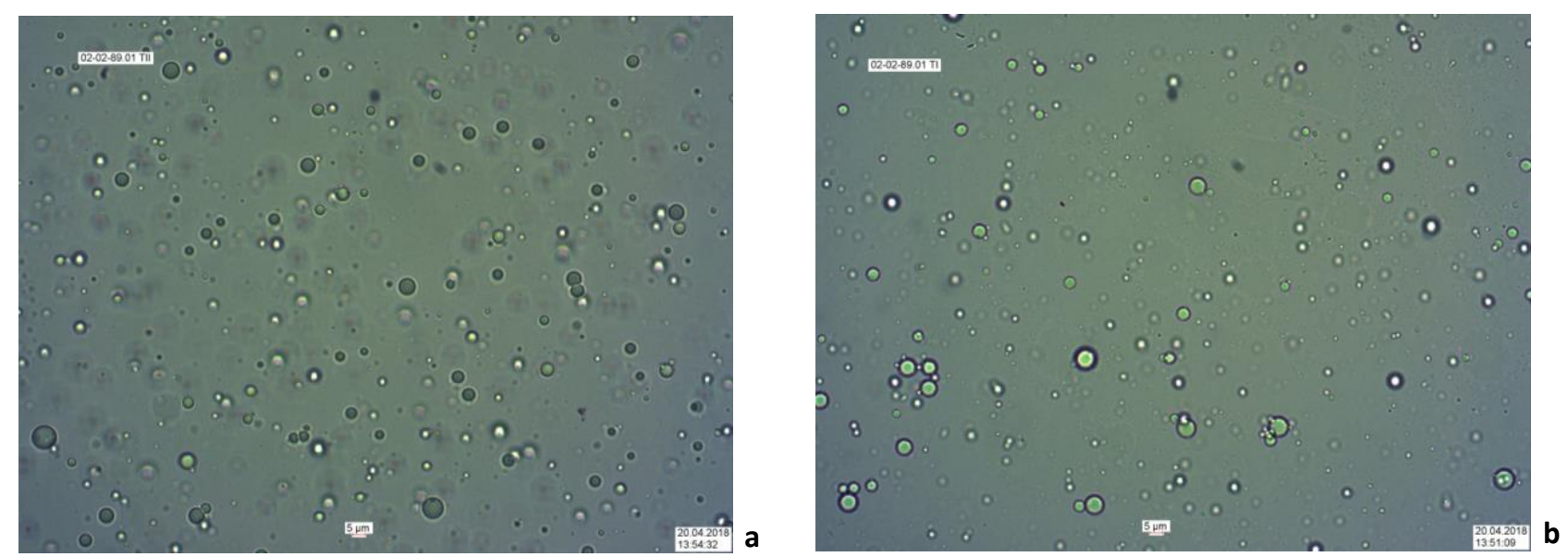

Figure 4-20: Oil droplets in unsheared [a] and strongly sheared [b] polymer solution 


\subsection{Sand-pack Flooding Tests}

The impact of pre-conditioning the polymer solution on the injectivity was investigated with flooding tests in sand-packed cells. First, the unsheared polymer solution was injected and its injection behavior is studied. Then, the injectivity of the polymer solutions sheared at different shearing intensities and with different devices, is examined. Following this, its improvement is analyzed. Further, it is studied whether the observed master curves between the viscosity and the screen factor of different shearing origins and intensities can also be confirmed on the pore-scale level. The shear rate in the sand-pack can be determined with Equation 2-19 at around 200 rps with $\alpha=2.5$, depending on the flow factor, $n$. This means the viscosity is in the shear thinning regime.

\subsubsection{Injection of Unconditioned Polymer Solution}

The injection result of the unsheared polymer solution in a 1,000 mD sand-pack cell is shown in Figure 4-21. The polymer flood was performed as described in the procedure above. The sand-pack is flooded by SSW-A at a rate of $3 \mathrm{ml} / \mathrm{min}$ for $14 \mathrm{PV}(\approx 30 \mathrm{~min})$. Following this, the injection rate of $S S W-A$ is reduced to $0.5 \mathrm{ml} / \mathrm{min}$ for $2 P V$ ( $\approx 30 \mathrm{~min}$ ). Figure 4-21 only shows the injection of the $0.5 \mathrm{ml} / \mathrm{min}$ for $2 P V$, and thus does not show the water breakthrough after the first $P V$ injected. As there is no occurrence of adsorption or other permeability reduction phenomena during the water injection, the $R F$ remains constant at 1 . After the injection of SSW-A, the polymer flood of PS-A begins. At $1 P V$ of polymer injection, a sharp bend occurs in the $R F$-curve, which marks the polymer breakthrough. After the polymer breakthrough, the $R F$ grows in a convex fashion, approaching an asymptotic value. At $26 P V$, the polymer injection is stopped, and SSW-A is injected again to determine the $R R F$ and thus to observe the permeability reduction caused due to polymer retention. The $R R F$ drops for a duration of $1 P V$ after switching to the injection of brine water. It then increases again with a similar convex shape during the polymer injection.

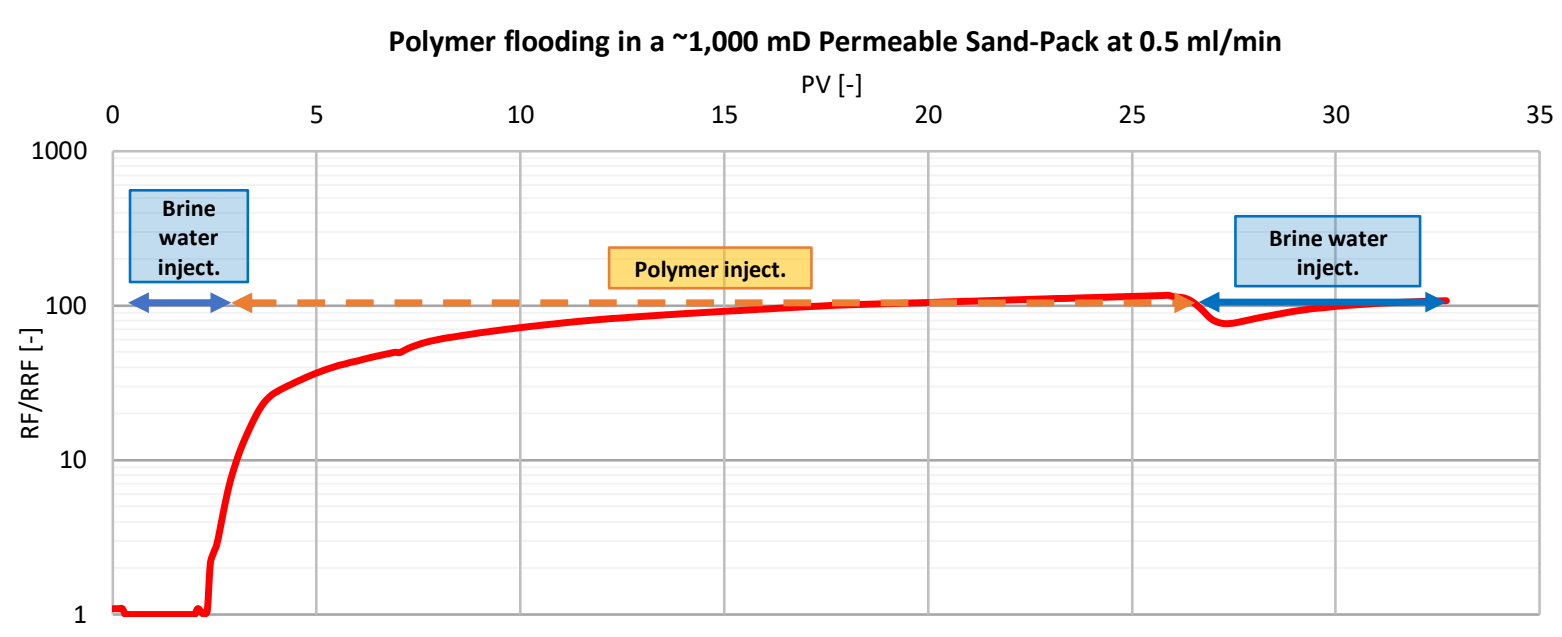

Figure 4-21: Polymer flood of unsheared polymer solution

The surface tension of the unsheared polymer solution was measured to be $25.3 \pm 0.5 \mathrm{mN} / \mathrm{m}$ and $25.4 \pm 0.5 \mathrm{mN} / \mathrm{m}$ before and after the sand injection with the measuring device KRUSS K12 Mk5 Processor Tensiometer. To investigate if the phenomenon of the increase in the convex $R F$ and $R R F$, for both the polymer injection and the water injection after the polymer flood, is owed to the amount of surfactant in the polymer system, injectivity tests with a higher and a lower surfactant concentration are performed. 
The same polymer solution is prepared and stored for 12 days in a separator funnel to observe the injectivity behavior for a polymer system with reduced inverter surfactant. A part of the oil phase separates itself from the solution after a few days due to coalescence, and it rises to the surface. The same occurs for the inverter surfactant. While the fresh solution is milky-like, the solution clears up after a few days. As micelles absorb light and are responsible for the milky color, the clearing up of the solution indicates that the surfactants present in the first few days also partly rise up to the surface. The entire polymer solution is separated into three parts using the separator funnel, whereby the lowest $20 \%$ and the upper $5 \%$ of the polymer solution in the separator funnel are separated from the middle part. Each part is shown in Figure 4-22 with the corresponding rheological parameters in Table 4-2. It can be inferred that the upper part then contains the separated oil and a high amount of surfactants since the light of the laser pointer disperses in the polymer solution. The middle part is injected in the sand-pack. The surface tension of the middle part is determined to be $32.7 \pm$ $0.5 \mathrm{mN} / \mathrm{m}$, which is an increase of $30 \%$ compared to the fresh solution. The amount of oil floating on the surface can be estimated, leaving around $40 \%$ of the oil in the middle part, which is injected.
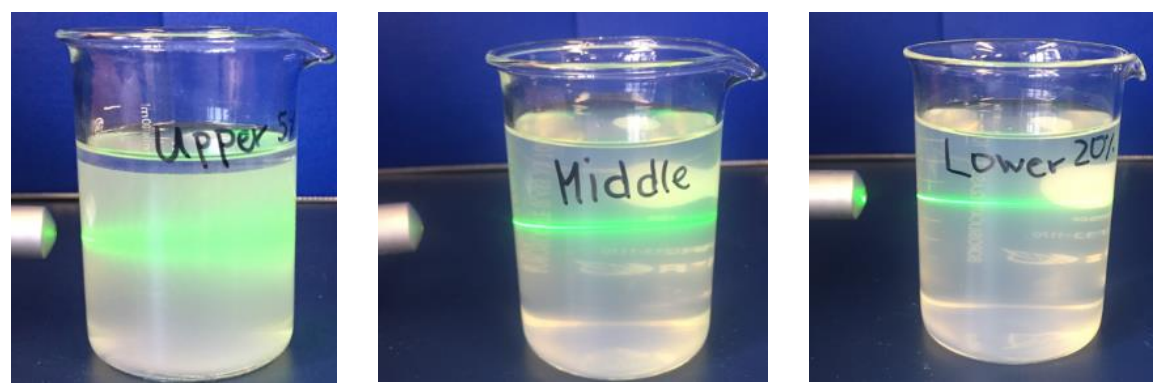

Figure 4-22: Unsheared polymer solution separated by density difference over 12 days

Table 4-2: Rheological parameters of unsheared separated polymer solution

\begin{tabular}{|c|c|c|c|c|}
\hline & & Upper 5\% & Middle 75\% & Lower 20\% \\
\hline $\mathrm{pH}( \pm 5 \%)$ & {$[-]$} & 7.1 & 6.9 & 7 \\
\hline Viscosity at $30^{\circ} \mathrm{C}$ from Ubbelohde $( \pm 5 \%)$ & [mPas] & 12.3 & 12.2 & 12.4 \\
\hline Viscosity at $150 \mathrm{rpm}$ at $30^{\circ} \mathrm{C}( \pm 5 \%)$ & [mPas] & 12.2 & 11.8 & 11.9 \\
\hline Screen factor ( $\pm 5 \%)$ & {$[-]$} & 20.3 & 20.5 & 20.3 \\
\hline
\end{tabular}

To observe the impact of an increased surfactant concentration, the surfactant concentration is increased from $2,400 \mathrm{ppm}$ to $3,500 \mathrm{ppm}$, while the polymer concentration is kept constant at 4,000 ppm. The rheological parameters of all three injections (polymer solution with 2,400 ppm surfactant, separated polymer solution with 2,400 ppm surfactant, and polymer solution with 3,500 ppm surfactant) are summarized in Table 4-3. The result of the injection is shown in Figure 4-23. 
Polymer Flooding in a 1,000 mD Permeable Sand-Pack Cell with 4,000 ppm Polymer EP-A

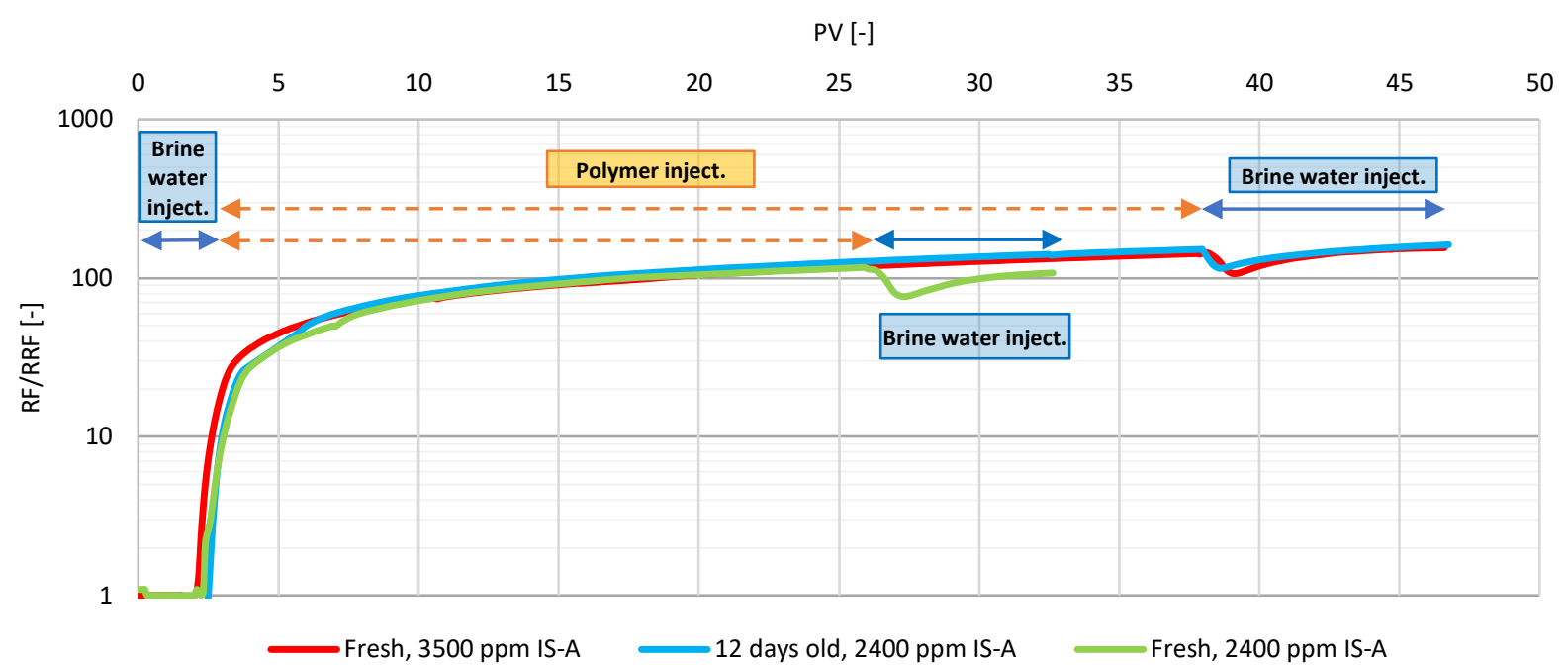

Figure 4-23: Polymer flooding test of unsheared polymer solution at different surfactant concentrations

Table 4-3: Rheological parameters of unsheared polymer solutions (I. with 2,400 ppm IS-A, II. with 2,400 ppm IS-A and then separated for several days, III. with 3,500 ppm IS-A)

\begin{tabular}{|c|c|c|c|c|}
\hline & & $\begin{array}{l}\text { I. polymer solution with } \\
2,400 \mathrm{ppm} I S-A\end{array}$ & $\begin{array}{l}\text { II. separated middle part of polymer } \\
\text { solution with } 2,400 \mathrm{ppm} / S-A\end{array}$ & $\begin{array}{l}\text { III. polymer solution with } \\
3,500 \mathrm{ppm} I S-A\end{array}$ \\
\hline $\mathrm{pH}( \pm 5 \%)$ & {$[-]$} & 7.4 & 6.9 & 7.4 \\
\hline $\begin{array}{l}\text { Viscosity at } 30^{\circ} \mathrm{C} \text { from Ubbelohde } \\
( \pm 5 \%)\end{array}$ & [mPas] & 12.5 & 12.2 & 12.4 \\
\hline $\begin{array}{l}\text { Viscosity at } 150 \mathrm{rpm} \text { at } 30^{\circ} \mathrm{C} \\
( \pm 5 \%)\end{array}$ & [mPas] & 12.2 & 11.8 & 12.4 \\
\hline Screen factor $( \pm 5 \%)$ & {$[-]$} & 18.4 & 20.5 & 18.8 \\
\hline
\end{tabular}

The $R F / R R F$ curves show a similar result for all three polymer solutions. Only a small variation occurs due to slight difference in permeability, measuring error, and other factors. The result shows that the amount of surfactant does not influence the injectivity. Also, a reduction in oil concentration does not make a remarkable difference, which shows that the impact of the oil droplets does not seem to be very significant.

The drop in the $R R F$ in the first $P V$ of water injection after polymer flooding can be explained by the smaller water molecules flowing through small pore throats that are inaccessible to polymers. After the injection of $1 P V$ of brine water, the $R R F$ again increases in a convex manner. This could be due to the cumulative blocking of pore throats by the trapped polymer chains dragged along with the water. Further analysis should be made in future work using measuring devices such as micro-CT scan.

\subsubsection{Device-specific Impact of Pre-conditioning on Injectivity}

In this section, the impact of pre-conditioning on injectivity for each shearing device is discussed. For all pre-sheared polymer floods, the following is applicable: After polymer breakthrough, the $R F$ increases continuously until water is injected. At 68 PV (except at 39 PV for capillary-sheared solution), the polymer injection is stopped, and brine water is injected, which leads to a significant drop in the pressure and thus a drop in the $R R F$. After the drop in the $R R F$, the $R R F$ remains at a constant value. The $R F$ increases sharply during the first $P V$ of polymer injection, followed by a sharp bend at the polymer breakthrough. After the breakthrough of polymer, the $R F$ increases linearly until SSW-A is injected. The filtration ratio $F R$ is the first indicator of the success of the injection. While the unsheared polymer solution has an $F R$ 
of 1.6, the sheared polymer solutions are all in the optimal range of 1.0 to 1.2 . It should be noted that the injection of the unsheared polymer solution only lasts for $42 \mathrm{PV}$, since the upper limit of the pressure sensor was reached.

\section{a) Injection of Disperser-sheared Polymer Solution}

The comparison between the unsheared and the disperser-sheared polymer solutions is shown in Figure 4-24. The sheared polymer solution was stirred with the disperser for $30 \mathrm{~min}$ at $1750 \mathrm{rpm}$ before being injected. The imposed energy input by the disperser of $14.6 \mathrm{MJ} / \mathrm{m}^{3}$, reduces the viscosity from 12.5 to $12 \mathrm{mPas}$ and the screen factor from 18.4 to 13.0 . The injectivity is significantly improved by $231 \%$, from 3.7 to 1.6 , after $30 P V$. When injecting SSW-A after the injection of the polymer solution, the $R R F$ is significantly lower than the $R F$ during polymer flooding. Nevertheless, polymer retention is still observed during the injection of the disperser-sheared polymer solution, which can be seen in an increase in the $R F$ during polymer injection and a significant difference between the $R R F$ and the $R F$ before polymer injection. The rheological results of the unsheared and the disperser-sheared polymer solution are shown in Table 4-4.

Polymer Flooding in a 1,000 mD Permeable Sand-Pack with Unsheared and Disperser-sheared Polymer Solutions

\section{PV [-]}

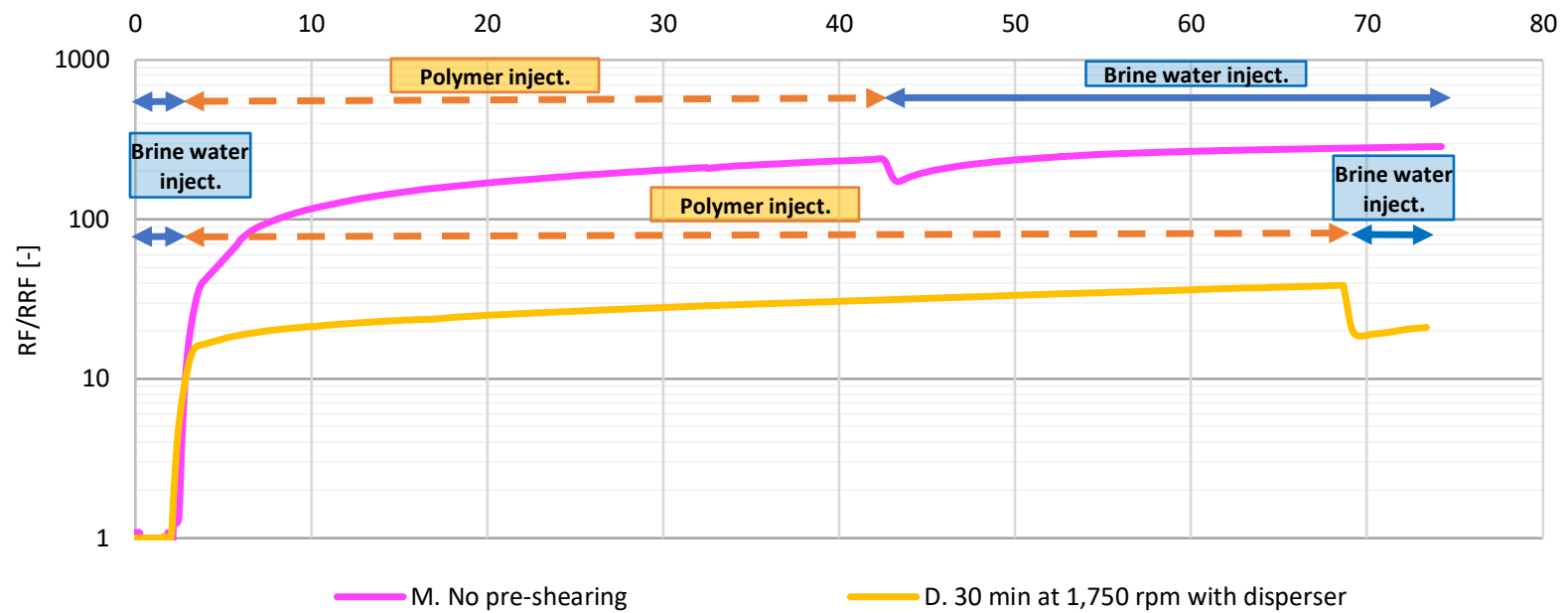

Figure 4-24: Injectivity results of unsheared and disperser-sheared polymer solutions

Table 4-4: Rheological results of injected polymer solutions - unsheared and disperser-sheared, referring to Figure 4-24

\begin{tabular}{|c|c|c|c|c|c|c|c|c|c|c|c|c|}
\hline & \multirow{2}{*}{$\begin{array}{c}\text { Volume } \\
\text { specific } \\
\text { energy } \\
\text { input }\end{array}$} & \multirow{2}{*}{$\begin{array}{c}\text { Viscosity at } \\
30^{\circ} \mathrm{C} \text { from } \\
\text { Ubbelohde } \\
( \pm 5 \%)\end{array}$} & \multirow{2}{*}{$\begin{array}{c}\text { Viscosity } \\
\text { at } 150 \text { rps } \\
( \pm 5 \%)\end{array}$} & \multirow{2}{*}{$\begin{array}{l}\text { Screen } \\
\text { factor } \\
( \pm 5 \%)\end{array}$} & \multirow{2}{*}{$\begin{array}{c}\mathrm{pH} \\
( \pm 5 \%)\end{array}$} & \multirow{2}{*}{$\begin{array}{c}\text { Filtration } \\
\text { ratio (FR) } \\
( \pm 5 \%)\end{array}$} & \multicolumn{3}{|c|}{$\begin{array}{c}\text { Resistance factor (RF) } \\
( \pm 0.5 \%)\end{array}$} & \multicolumn{2}{|c|}{$\begin{array}{l}\text { Injection gradient } \\
\text { (IG) }( \pm 0.5 \%)\end{array}$} & \multirow{2}{*}{$\begin{array}{c}\text { Residual } \\
\text { resistance } \\
\text { factor } \\
(R R F) \\
( \pm 0.5 \%)\end{array}$} \\
\hline & & & & & & & $\begin{array}{l}R F \text { at } \\
3 P V\end{array}$ & $\begin{array}{l}R F \text { at } \\
30 P V\end{array}$ & $\begin{array}{l}R F \text { at } \\
60 P V\end{array}$ & $\begin{array}{l}I G \text { at } \\
30 P V\end{array}$ & $\begin{array}{l}1 G \text { at } \\
60 P V\end{array}$ & \\
\hline & {$[\mathrm{MJ} / \mathrm{m} 3]$} & [mPas] & [mPas] & {$[-]$} & {$[-]$} & {$[-]$} & {$[-]$} & {$[-]$} & {$[-]$} & {$[-]$} & {$[-]$} & {$[-]$} \\
\hline$M$ & 0 & 12.5 & 12.2 & 18.4 & 7.4 & 1.6 & 57.3 & 210.1 & - & 3.7 & - & $>>100$ \\
\hline D & 14.6 & 12.0 & 12.0 & 13.0 & 7.8 & 1.08 & 17.9 & 28.6 & 36.8 & 1.6 & 1.3 & 21.8 \\
\hline
\end{tabular}

\section{b) Injection of Turrax-sheared Polymer Solutions}

The injection results of the turrax-sheared polymer solutions and the unsheared polymer solution are shown in Figure 4-25 and Table 4-5. Solution T1 was sheared for $1 \mathrm{~min}$ at 20,000 rpm, solution T2 for $7.75 \mathrm{~min}$ at 10,000 rpm, and solution T3 for $7.75 \mathrm{~min}$ at 20,000 rpm. The rheological results are summarized in Table 4-5. Also, here, the turrax-sheared polymer solutions show a significant improvement in injectivity. Solutions T2 and T3 show an improving injectivity with the increasing shear intensity. Solution $\mathrm{T} 2$ still shows polymer retention due to an increasing $R F$ and a high $R R F$. Solution T3 
shows a favorable injectivity behavior with nearly no retention of polymer, giving an IG of 1.1. The comparably poor injectivity behavior of solution T1 can be explained by the insufficient residence time of the turrax. This means that even though the overall viscosity and the screen factor dropped enough to expect a favorable injectivity behavior, the entire solution was not sheared in 1 minute as explained in section 4.2.6. Thus, it can be assumed that a few too-high molecular chains were left unruptured causing an increased mechanical trapping behavior, which cannot be detected by the viscometers or rheometers.

Polymer Flooding in a 1,000 mD Permeable Sand-Pack with Unsheared and Turrax-sheared Polymer Solutions PV [-]

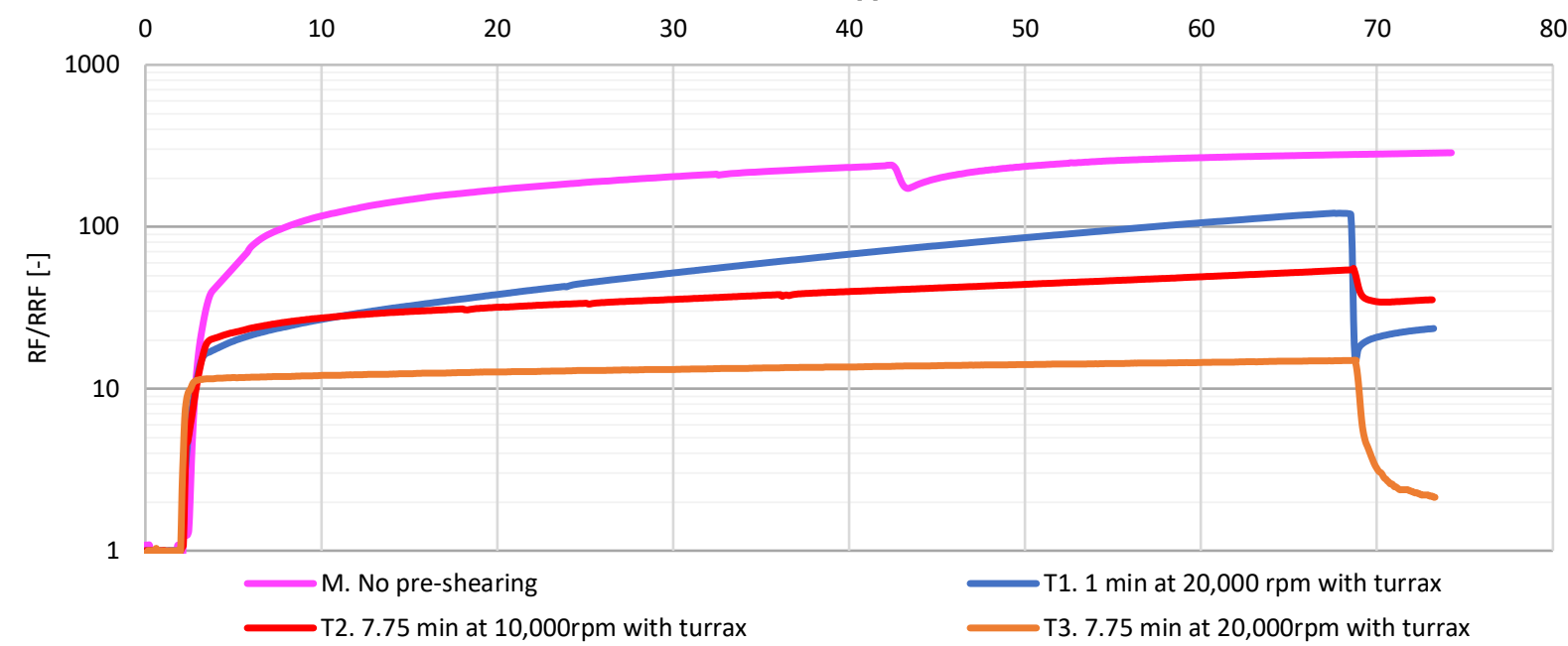

Figure 4-25: Injectivity results of unsheared and turrax-sheared polymer solutions

Table 4-5: Rheological results of injected polymer solutions - unsheared and turrax-sheared, referring to Figure 4-25

\begin{tabular}{|c|c|c|c|c|c|c|c|c|c|c|c|c|}
\hline & \multirow{2}{*}{$\begin{array}{c}\text { Volume } \\
\text { specific } \\
\text { energy } \\
\text { input }\end{array}$} & \multirow{2}{*}{$\begin{array}{c}\text { Viscosity at } \\
30^{\circ} \mathrm{C} \text { from } \\
\text { Ubbelohde } \\
( \pm 5 \%)\end{array}$} & \multirow{2}{*}{$\begin{array}{c}\text { Viscosity } \\
\text { at } 150 \mathrm{rps} \\
( \pm 5 \%)\end{array}$} & \multirow{2}{*}{$\begin{array}{l}\text { Screen } \\
\text { factor } \\
( \pm 5 \%)\end{array}$} & \multirow{2}{*}{$\begin{array}{c}\mathrm{pH} \\
( \pm 5 \%)\end{array}$} & \multirow{2}{*}{$\begin{array}{c}\text { Filtration } \\
\text { ratio }(F R) \\
( \pm 5 \%)\end{array}$} & \multicolumn{3}{|c|}{$\begin{array}{l}\text { Resistance factor (RF) } \\
( \pm 0.5 \%)\end{array}$} & \multicolumn{2}{|c|}{$\begin{array}{l}\text { Injection gradient } \\
(I G)( \pm 0.5 \%)\end{array}$} & \multirow{2}{*}{$\begin{array}{c}\text { Residual } \\
\text { resistance } \\
\text { factor } \\
\text { (RRF) } \\
( \pm 0.5 \%)\end{array}$} \\
\hline & & & & & & & $\begin{array}{l}R F \text { at } \\
3 P V\end{array}$ & $\begin{array}{l}R F \text { at } \\
30 P V\end{array}$ & $\begin{array}{l}R F \text { at } \\
60 P V\end{array}$ & $\begin{array}{l}\text { IG at } \\
30 P V\end{array}$ & $\begin{array}{l}\text { IG at } \\
60 P V\end{array}$ & \\
\hline & {$[\mathrm{MJ} / \mathrm{m} 3]$} & [mPas] & [mPas] & {$[-]$} & {$[-]$} & {$[-]$} & {$[-]$} & {$[-]$} & {$[-]$} & {$[-]$} & {$[-]$} & {$[-]$} \\
\hline$M$ & 0 & 12.5 & 12.2 & 18.4 & 7.4 & 1.6 & 57.3 & 210.1 & - & 3.7 & - & $>>100$ \\
\hline T1 & 36.8 & 11.1 & 11.2 & 11.1 & 7.4 & 1.13 & 12.6 & 51.9 & 106.0 & 4.1 & 2.0 & 27.0 \\
\hline $\mathrm{T} 2$ & 31.3 & 11.1 & 11.1 & 10.3 & 7.3 & 1.07 & 22.3 & 36.5 & 50.3 & 1.6 & 1.4 & 35.6 \\
\hline T3 & 290.7 & 9.3 & 9.5 & 6.4 & 7.5 & 1.11 & 11.7 & 13.3 & 14.6 & 1.1 & 1.1 & 2.0 \\
\hline
\end{tabular}

\section{c) Injection of Capillary-sheared Polymer Solutions}

Solution $\mathrm{C}$ was sheared significantly by forcing the solution through a 1-mm-thin capillary tube with a length of $10 \mathrm{~cm}$ at a pressure of 20 bar. The injection with the corresponding data is shown in Figure 4-26 and Table 4-6. The viscosity is reduced from 12.5 to $10.4 \mathrm{mPas}$, and the screen factor is reduced from 18.4 to 8.7. Still, the injectivity test shows high polymer retention, indicating that the polymer has not been sheared is not sufficiently. This implies that pumping the polymer through an orifice or valve causes a significant mechanical degradation. However, it changes the polymer structure in a different way than the agitators do. Interestingly, the $F R$ of $C$ is 1.05 , which indicates a favorable injectivity behavior, which shows that the $F R$ can only be taken as an indicator for a favorable injectivity behavior. Nevertheless, flooding tests are inevitable to make an exact statement on the injectability. 
Polymer Flooding in a 1,000 mD Permeable Sand-Pack with Unsheared and Capillary-sheared Polymer Solutions

$$
\text { PV [-] }
$$

0

10

20

30

40

50

60

70

80

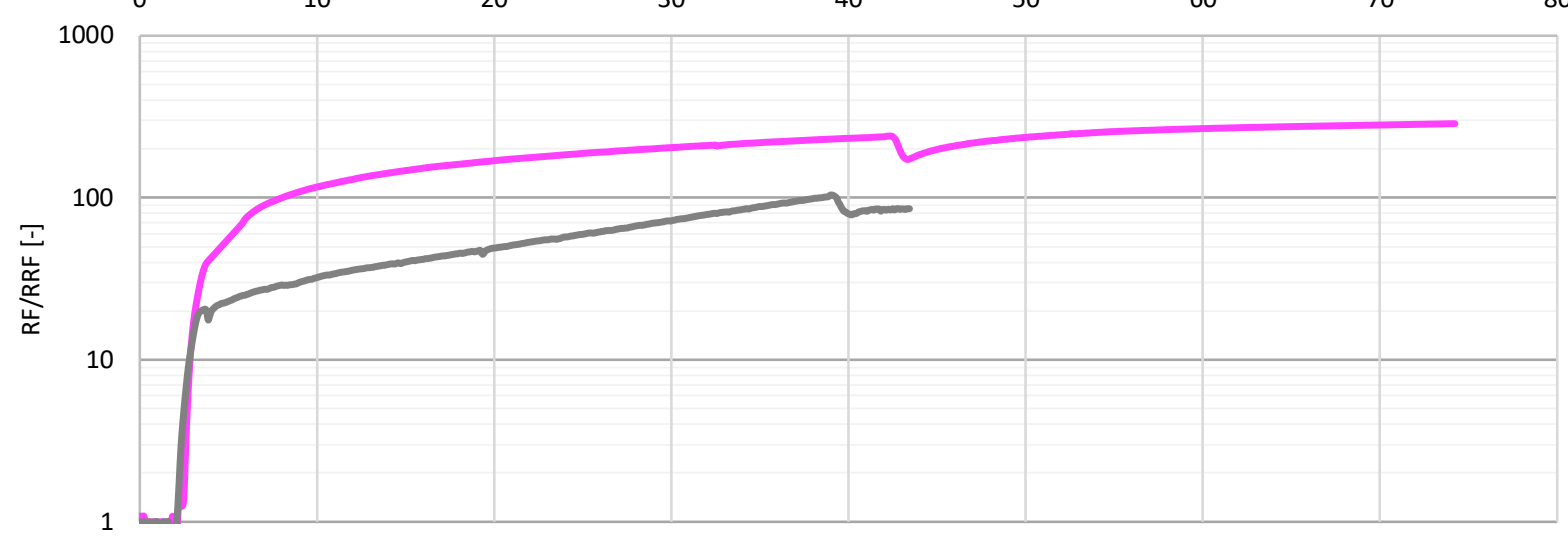

M. No pre-shearing

C. force through $1 \mathrm{~mm}$ thinn $10 \mathrm{~cm}$ long capillary at 20 bar

Figure 4-26: Injectivity results of unsheared and capillary-sheared polymer solutions

Table 4-6: Rheological results of injected polymer solutions - unsheared and turrax-sheared, referring to Figure 4-26

\begin{tabular}{|c|c|c|c|c|c|c|c|c|c|c|c|c|}
\hline & \multirow{2}{*}{$\begin{array}{c}\text { Volume } \\
\text { specific } \\
\text { energy } \\
\text { input }\end{array}$} & \multirow{2}{*}{$\begin{array}{c}\text { Viscosity at } \\
30^{\circ} \mathrm{C} \text { from } \\
\text { Ubbelohde } \\
( \pm 5 \%)\end{array}$} & \multirow{2}{*}{$\begin{array}{c}\text { Viscosity } \\
\text { at } 150 \\
\text { rps } \\
( \pm 5 \%)\end{array}$} & \multirow{2}{*}{$\begin{array}{l}\text { Screen } \\
\text { factor } \\
( \pm 5 \%)\end{array}$} & \multirow{2}{*}{$\begin{array}{c}\mathrm{pH} \\
( \pm 5 \%)\end{array}$} & \multirow{2}{*}{$\begin{array}{c}\text { Filtration } \\
\text { ratio (FR) } \\
( \pm 5 \%)\end{array}$} & \multicolumn{3}{|c|}{$\begin{array}{l}\text { Resistance factor (RF) } \\
( \pm 0.5 \%)\end{array}$} & \multicolumn{2}{|c|}{$\begin{array}{l}\text { Injection gradient } \\
(I G)( \pm 0.5 \%)\end{array}$} & \multirow{2}{*}{$\begin{array}{c}\text { Residual } \\
\text { resistance } \\
\text { factor } \\
(R R F) \\
( \pm 0.5 \%)\end{array}$} \\
\hline & & & & & & & $\begin{array}{l}R F \text { at } \\
3 P V\end{array}$ & $\begin{array}{l}R F \text { at } \\
30 P V\end{array}$ & $\begin{array}{l}R F \text { at } \\
60 P V\end{array}$ & $\begin{array}{l}I G \text { at } \\
30 P V\end{array}$ & $\begin{array}{l}I G \text { at } \\
60 P V\end{array}$ & \\
\hline & {$[\mathrm{MJ} / \mathrm{m} 3]$} & [mPas] & [mPas] & {$[-]$} & {$[-]$} & {$[-]$} & {$[-]$} & {$[-]$} & {$[-]$} & {$[-]$} & {$[-]$} & {$[-]$} \\
\hline$M$ & 0 & 12.5 & 12.2 & 18.4 & 7.4 & 1.6 & 57.3 & 210.1 & - & 3.7 & - & $\gg>100$ \\
\hline C & 50.1 & 10.1 & 10.4 & 8.7 & 7.5 & 1.05 & 14.0 & 79.0 & - & 5.7 & - & 35.6 \\
\hline
\end{tabular}

\section{d) Injection of Disperser-Turrax-sheared Polymer Solutions}

The disperser-sheared polymer solution shows a significantly improved injectivity behavior, as compared to the unsheared polymer solution, by reducing the viscosity by only $1.6 \%$. Compared to this, the turrax-sheared polymer solution reduced the viscosity to a larger extent at a comparable injection behavior. Now, the polymer solution was first sheared with the disperser for $30 \mathrm{~min}$ at $1750 \mathrm{rpm}$, and it was then sheared with a turrax at different intensities. The injection results of the unsheared, disperser-sheared, and disperser-turrax-sheared polymer solutions are shown in Figure 4-27 with the additional data given in Table 4-7.

The sequence of the injections shown in Figure 4-27 goes from unsheared to highly sheared, meaning that the $R F$ reduces with the shear intensity. The slope of the increase in the $R F$ reduces with shear intensity. This means that the more the solution is sheared, the closer the $I G$ gets to the ideal value of 1 . By increasing the shear intensity and improving $I G$, both the screen factor and viscosity reduce. While the viscosity is reduced by a maximum of $20 \%$ (compare solutions $M$ and DT4), the screen factor is again more sensitive to pre-shearing, and it is reduced by a maximum of $57 \%$ (compare solutions $\mathrm{M}$ and $\mathrm{DT} 4)$. The $R R F$ and thus residual permeability reduction, likewise, reduce with the shearing intensity. 
Polymer Flooding in a 1,000 mD Permeable Sand-Pack with Unsheared and Disperser-turrax-sheared Polymer Solutions

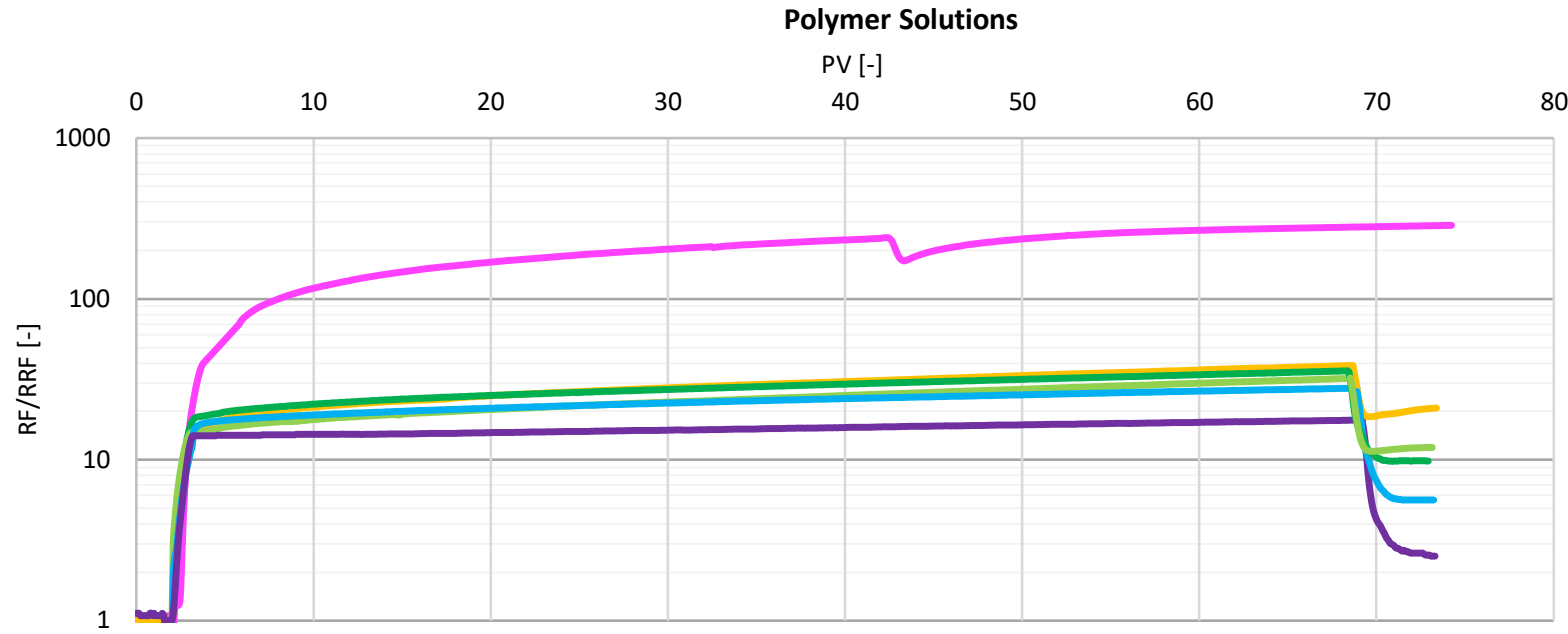

M. No pre-shearing

D. $30 \mathrm{~min}$ at $1,750 \mathrm{rpm}$ with disperser

DT1. $30 \mathrm{~min}$ at $1,750 \mathrm{rpm}$ with disperser, followed by $5.5 \mathrm{~min}$ at $10,000 \mathrm{rpm}$ with turrax

DT2. $30 \mathrm{~min}$ at 1,750 rpm with disperser, followed by $1 \mathrm{~min}$ at 20,000 rpm with turrax

DT3. $30 \mathrm{~min}$ at $1,750 \mathrm{rpm}$ with disperser, followed by $10 \mathrm{~min}$ at $10,000 \mathrm{rpm}$ with turrax

DT4. $30 \mathrm{~min}$ at $1,750 \mathrm{rpm}$ with disperser, followed by $5.5 \mathrm{~min}$ at $20,000 \mathrm{rpm}$ with turrax

Figure 4-27: Injectivity results of unsheared and disperser-turrax-sheared polymer solutions

Table 4-7: Rheological results of injected polymer solutions - unsheared, disperser-sheared and disperser-turrax-sheared, referring to Figure 4-27

\begin{tabular}{|c|c|c|c|c|c|c|c|c|c|c|c|c|}
\hline & \multirow{2}{*}{$\begin{array}{c}\text { Volume } \\
\text { specific } \\
\text { energy } \\
\text { input }\end{array}$} & \multirow{2}{*}{$\begin{array}{c}\text { Viscosity at } \\
30^{\circ} \mathrm{C} \text { from } \\
\text { Ubbelohde } \\
( \pm 5 \%)\end{array}$} & \multirow{2}{*}{$\begin{array}{l}\text { Viscosity } \\
\text { at } 150 \\
\text { rps } \\
( \pm 5 \%)\end{array}$} & \multirow{2}{*}{$\begin{array}{l}\text { Screen } \\
\text { factor } \\
( \pm 5 \%)\end{array}$} & \multirow{2}{*}{$\begin{array}{c}\mathrm{pH} \\
( \pm 5 \%)\end{array}$} & \multirow{2}{*}{$\begin{array}{c}\text { Filtration } \\
\text { ratio (FR) } \\
( \pm 5 \%)\end{array}$} & \multicolumn{3}{|c|}{$\begin{array}{c}\text { Resistance factor (RF) } \\
( \pm 0.5 \%)\end{array}$} & \multicolumn{2}{|c|}{$\begin{array}{l}\text { Injection gradient } \\
(I G)( \pm 0.5 \%)\end{array}$} & \multirow{2}{*}{$\begin{array}{c}\text { Residual } \\
\text { resistance } \\
\text { factor } \\
(R R F) \\
( \pm 0.5 \%)\end{array}$} \\
\hline & & & & & & & $\begin{array}{l}R F \text { at } \\
3 P V\end{array}$ & $\begin{array}{l}R F \text { at } \\
30 P V\end{array}$ & $\begin{array}{l}R F \text { at } \\
60 P V\end{array}$ & $\begin{array}{l}\text { IG at } \\
30 P V\end{array}$ & $\begin{array}{l}\text { IG at } \\
60 P V\end{array}$ & \\
\hline & {$[\mathrm{MJ} / \mathrm{m} 3]$} & [mPas] & [mPas] & {$[-]$} & {$[-]$} & {$[-]$} & {$[-]$} & {$[-]$} & {$[-]$} & {$[-]$} & {$[-]$} & {$[-]$} \\
\hline$M$ & 0 & 12.5 & 12.2 & 18.4 & 7.4 & 1.6 & 57.3 & 210.1 & - & 3.7 & - & $\gg 100$ \\
\hline D & 14.6 & 12.0 & 12 & 12.9 & 7.8 & 1.08 & 17.9 & 28.6 & 36.8 & 1.6 & 1.3 & 21.8 \\
\hline DT1 & 36.8 & 11.2 & 11.3 & 10.5 & $\begin{array}{l}7.8 \\
\end{array}$ & 1.09 & 19.6 & 27.7 & 34.2 & 1.4 & 1.2 & 9.6 \\
\hline DT2 & 52.1 & 11.3 & 11.1 & 10.9 & 7.8 & 1.07 & 16.0 & 23.3 & 30.4 & 1.5 & 1.3 & 11.7 \\
\hline DT3 & 55.3 & 10.5 & 10.8 & 9.3 & 7.8 & 1.04 & 17.6 & 22.8 & 27.0 & 1.3 & 1.2 & 5.6 \\
\hline DT4 & 220.9 & 9.9 & 9.9 & $\begin{array}{l}7.9 \\
\end{array}$ & 7.8 & 1.02 & 15.0 & 15.4 & 17.3 & 1.0 & 1.1 & 2.2 \\
\hline
\end{tabular}

As shown in Figure 4-28, only a small amount of pre-shearing improves the injectivity significantly. By pre-shearing the polymer solution with a volume specific energy input of $14.6 \mathrm{MJ} / \mathrm{m}^{3}$, the viscosity is reduced merely by $1.6 \%$, while the injectivity improves already by $190 \%$. As the screen factor drops by $30 \%$, this indicates that the polymer solution is strongly disentangled, without causing significant rupturing of the polymer chains. The more the polymer solution is then pre-sheared, the more the injectivity behavior improves, while the viscosity and screen factor further reduce. While the viscosity only reduces slowly, the screen factor, which indicates the transition of the polymer from the entangled to the stretched state, reduces more drastically. 


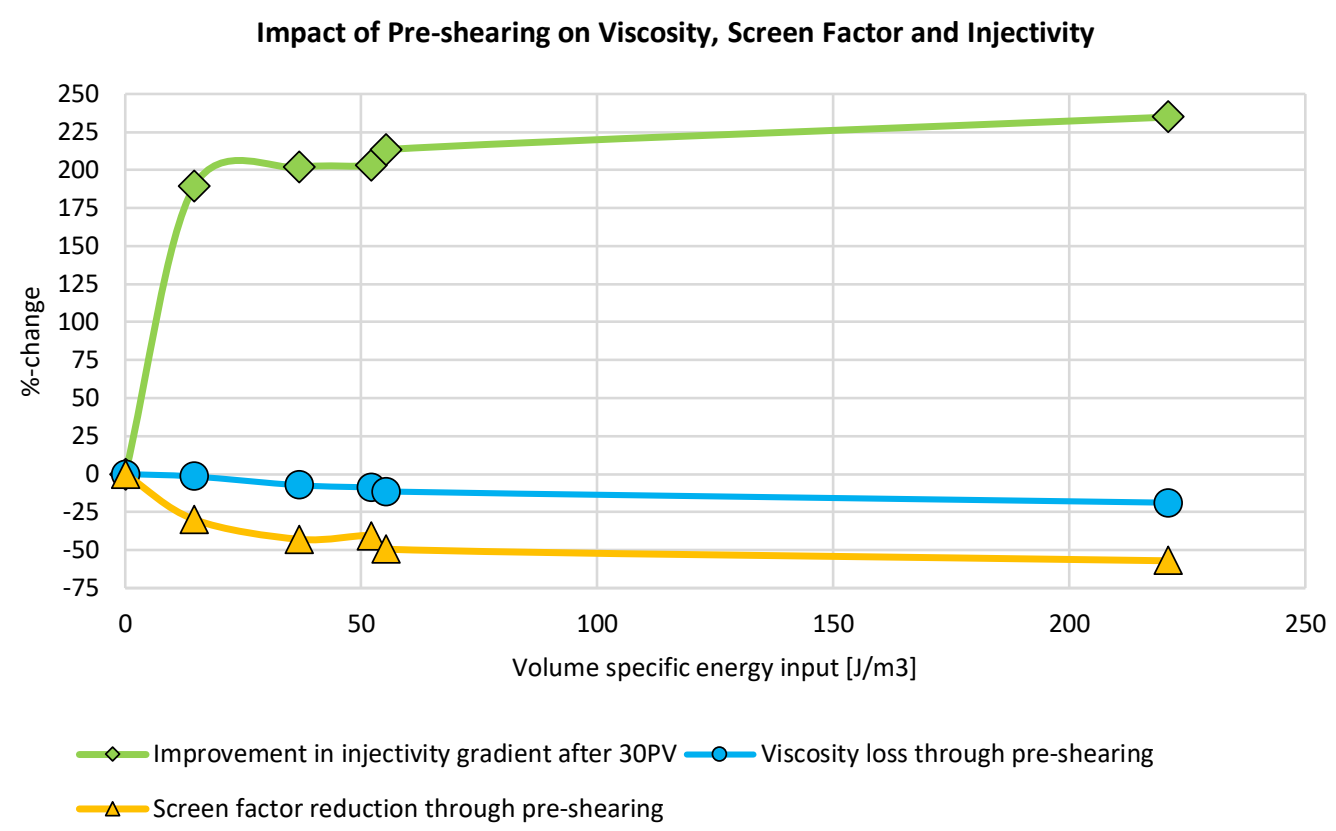

Figure 4-28: Impact of pre-shearing on viscosity, screen factor and injectivity

\subsubsection{Device-independent Impact of Pre-conditioning on Injectivity}

In section 4.3.2, the device-specific impact of pre-conditioning on injectivity was shown for disperser-, turrax-, capillary-, and disperser-turrax-sheared polymer solutions. In this section, the differently sheared polymer solutions are compared with each other, and it is investigated whether the master curves, introduced in section 4.3.1, also exist for injectivity. Further, the mechanical degradation and polymer retention for pre-sheared polymer solutions are analyzed.

\section{a) Comparison of Differently Sheared Polymer Solutions to Bulk Master Curve}

All conducted injection curves are shown in Figure 4-29 and Table 4-8, which are both sorted in the order of improving IGs. The disperser-sheared polymer solution (referred to as solution D) has a lower energy input as well as a higher viscosity and screen factor than the turrax-sheared polymer solutions, solutions $\mathrm{T} 2$ and $\mathrm{T} 3$, while solution $\mathrm{D}$ still shows a better injectivity behavior. Also, it should be noted, as explained in section 4.3.2 b), T1 does not correspond to the sequence of the turrax-sheared solutions, as the residence time was too low.

The pre-shearing with the flow through capillary under pressure does not lead to a favorable injectivity. This indicates that even though rheological master curves exist between the differently sheared polymer solutions, this strong link cannot be observed during injectivity when shearing is done with different devices. 
Polymer Flooding in a 1,000mD Permeable Sand-Pack with Unsheared and Differently Sheared Polymer Solutions

PV [-]

$\begin{array}{llll}40 & 50 & 60 & 70\end{array}$

70

80

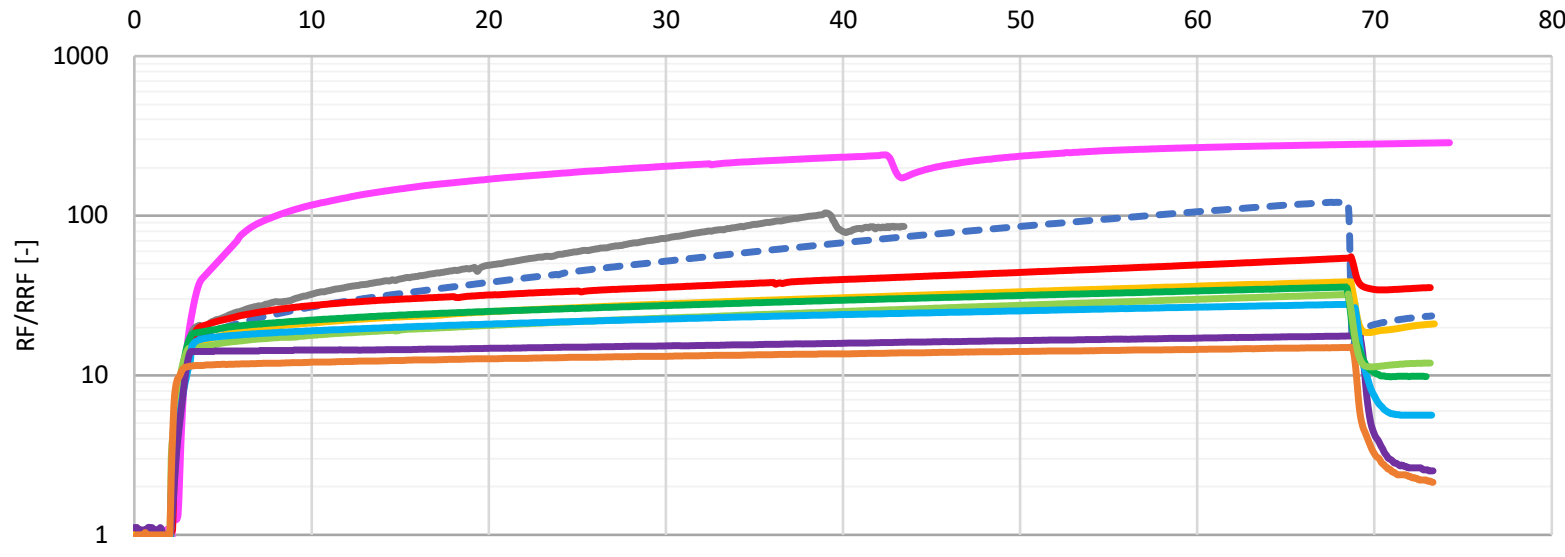

1

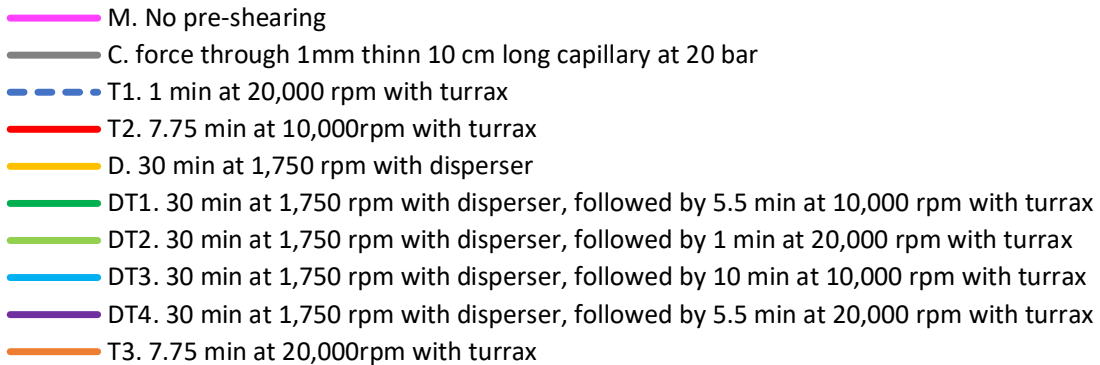

Figure 4-29: Injectivity results of unsheared and sheared polymer solutions of different shearing origins and intensities

Table 4-8: Rheological results of injected polymer solutions - unsheared and differently sheared, referring to Figure 4-29

\begin{tabular}{|c|c|c|c|c|c|c|c|c|c|c|c|c|}
\hline & \multirow{2}{*}{$\begin{array}{c}\text { Volume } \\
\text { specific } \\
\text { energy } \\
\text { input }\end{array}$} & \multirow{2}{*}{$\begin{array}{c}\text { Viscosity at } \\
30^{\circ} \mathrm{C} \text { from } \\
\text { Ubbelohde } \\
( \pm 5 \%)\end{array}$} & \multirow{2}{*}{$\begin{array}{c}\text { Viscosity } \\
@ 150 \\
\text { rps } \\
( \pm 5 \%)\end{array}$} & \multirow{2}{*}{$\begin{array}{l}\text { Screen } \\
\text { factor } \\
( \pm 5 \%)\end{array}$} & \multirow{2}{*}{$\begin{array}{c}\mathrm{pH} \\
( \pm 5 \%)\end{array}$} & \multirow{2}{*}{$\begin{array}{c}\text { Filtration } \\
\text { ratio }(F R) \\
( \pm 5 \%)\end{array}$} & \multicolumn{3}{|c|}{$\begin{array}{l}\text { Resistance factor (RF) } \\
( \pm 0.5 \%)\end{array}$} & \multicolumn{2}{|c|}{$\begin{array}{l}\text { Injection gradient } \\
(I G)( \pm 0.5 \%)\end{array}$} & \multirow{2}{*}{$\begin{array}{c}\text { Residual } \\
\text { resistance } \\
\text { factor } \\
\text { (RRF) } \\
\text { ( } \pm 0.5 \%)\end{array}$} \\
\hline & & & & & & & $\begin{array}{l}R F \text { at } \\
3 P V\end{array}$ & $\begin{array}{l}R F \text { at } \\
30 P V\end{array}$ & $\begin{array}{l}R F \text { at } \\
60 P V\end{array}$ & $\begin{array}{l}I G \text { at } \\
30 P V\end{array}$ & $\begin{array}{l}\text { IG at } \\
60 P V\end{array}$ & \\
\hline & {$[\mathrm{MJ} / \mathrm{m} 3]$} & [mPas] & [mPas] & {$[-]$} & {$[-]$} & [-] & {$[-]$} & {$[-]$} & [-] & {$[-]$} & {$[-]$} & {$[-]$} \\
\hline $\mathbf{M}$ & 0 & 12.5 & 12.2 & 18.4 & 7.4 & 1.6 & 57.3 & 210.1 & - & 3.7 & - & $>>100$ \\
\hline C & 50.1 & 10.1 & 10.4 & 8.7 & 7.5 & 1.05 & 14.0 & 79.0 & - & 5.7 & - & 35.6 \\
\hline T1 & 36.8 & 11.1 & 11.2 & 11.1 & 7.4 & 1.13 & 12.6 & 51.9 & 106.0 & 4.1 & 2.0 & 27.0 \\
\hline T2 & 31.3 & 11.1 & 11.1 & 10.3 & 7.3 & 1.07 & 22.3 & 36.5 & 50.3 & 1.6 & 1.4 & 35.6 \\
\hline D & 14.6 & 12 & 12 & 12.9 & 7.8 & 1.08 & 17.9 & 28.6 & 36.8 & 1.6 & 1.3 & 21.8 \\
\hline DT1 & 36.8 & 11.2 & 11.3 & 10.5 & 7.8 & 1.09 & 19.6 & 27.7 & 34.2 & 1.4 & 1.2 & 9.6 \\
\hline DT2 & 52.1 & 11.3 & 11.1 & 10.9 & 7.8 & 1.07 & 16.0 & 23.3 & 30.4 & 1.5 & 1.3 & 11.7 \\
\hline DT3 & 55.3 & 10.5 & 10.8 & 9.3 & 7.8 & 1.04 & 17.6 & 22.8 & 27.0 & 1.3 & 1.2 & 5.6 \\
\hline DT4 & 220.9 & 9.9 & 9.9 & 7.9 & 7.8 & 1.02 & 15.0 & 15.4 & 17.3 & 1.0 & 1.1 & 2.2 \\
\hline T3 & 290.7 & 9.3 & 9.5 & 6.4 & 7.5 & 1.11 & 11.7 & 13.3 & 14.6 & 1.1 & 1.1 & 2.0 \\
\hline
\end{tabular}

Figure 4-30 visualizes the viscosity and screen factor dependency of each injection and shows the corresponding IG. The figure emphasizes that even though there is a strong viscosity-screen factor dependency, the IG does not correspond to this. The IG corresponds only within one shearing device assuming the residence time is sufficient. 


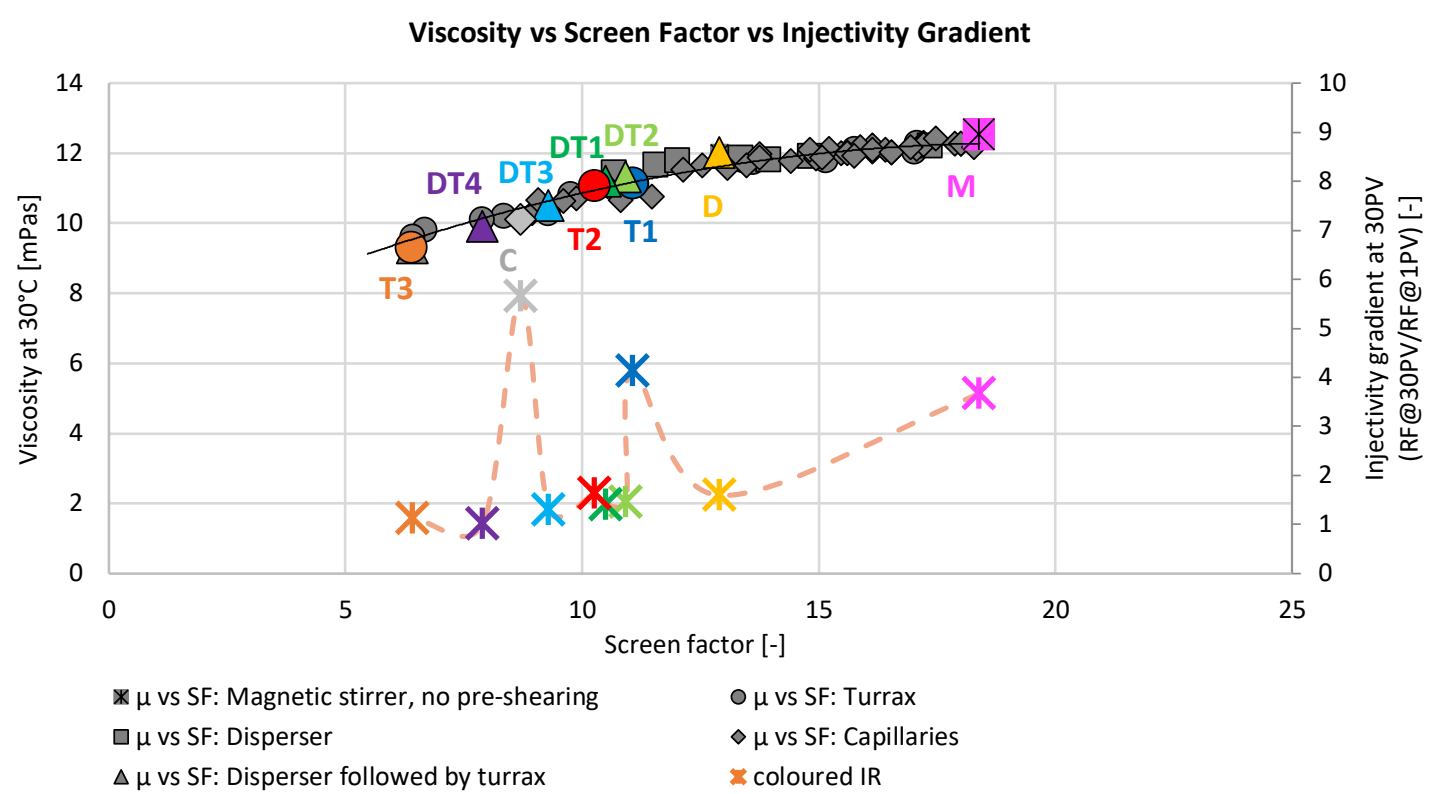

Figure 4-30: Viscosity from Ubbelohde and injectivity gradient after $30 \mathrm{PV}$ of polymer-injected versus screen factor of carried out injections

As it has been already discussed in section 4.3.1, the $\mathrm{pH}$ variations occur depending on the shearing device and intensity. It can be implied that with a higher $\mathrm{pH}$, a better injectivity can be reached by comparing solutions $\mathrm{D}$ and T2. Solution $\mathrm{D}$ shows a better injectivity at a higher viscosity with a 7\% higher $\mathrm{pH}$ as compared to solution $\mathrm{T} 2$, which shows a slightly poorer injectivity at a lower viscosity. This indicates that at a higher $\mathrm{pH}$, the injectivity gives a better result. The dependency on the $\mathrm{pH}$ is inferred in the following paragraph.

To investigate on the influence of $\mathrm{pH}$ on the injectivity, an unsheared and a sheared solution are injected with an adjusted $\mathrm{pH}$ value. Figure 4-31a and the corresponding Table 4-9 show the comparison of the same unsheared polymer solution having different $\mathrm{pH}$. The $\mathrm{pH}$ of solution $\mathrm{M}$ is 7.4 and not adjusted, and the $\mathrm{pH}$ of solution $\mathrm{M}-1$ is adjusted from 7.4 to 8.1. Figure 4-31b and the corresponding Table 4-10 show the same change in pH, but it is for the turrax-sheared polymer solution. The $\mathrm{pH}$ adjustment to a more basic milieu improves the injectivity significantly. The polymer retention can be reduced, which can be seen by a significant reduction in $R R F$. This can be explained by a reduced polymer adsorption. At a higher $\mathrm{pH}$, the sand surfaces becomes more negative. The negatively charged groups, such as carboxyl groups in the polymer chain prevents the adsorption on the sand surface (Saurabh, Achinta, \& Mandal, 2013). 


\section{Impact of pH Adjustment on Polymer Flooding in 1000 mD Sand-Pack of Unsheared Polymer} Solution

PV [-]

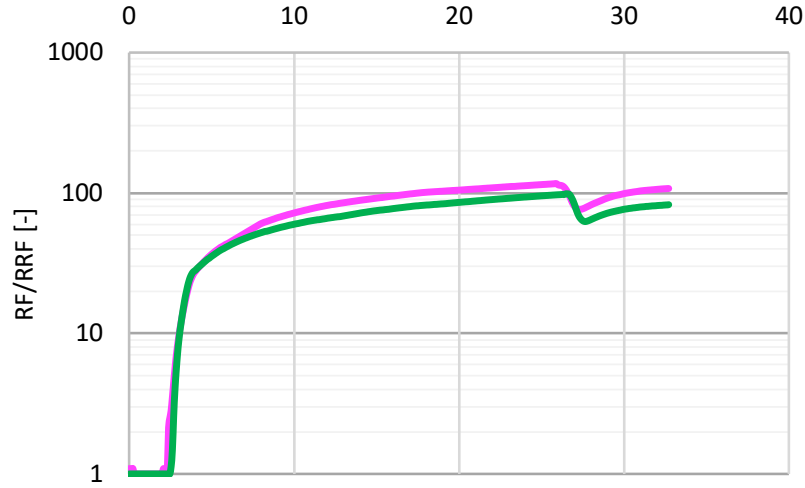

M: without $\mathrm{pH}$ adjustment

M-1: with pH adjustment to 8.0
Impact of pH Adjustment on Polymer Flooding in 1000 mD Sand-Pack with Turrax-sheared Polymer Solution

PV [-]

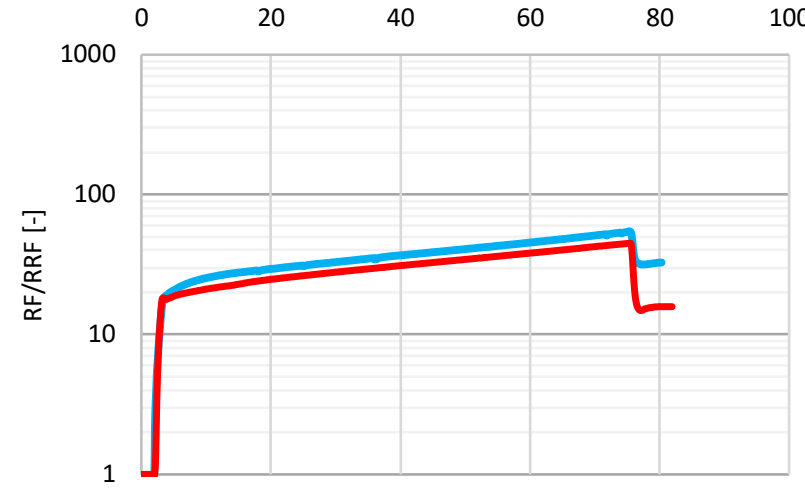

T2: without $\mathrm{pH}$ adjustment

a $\quad$ T2-1: with $\mathrm{pH}$ adjustment to 8.0

100

Figure 4-31: Injectivity results of polymer solutions of the same unsheared polymer solution, but different pH adjustment ( $\mathrm{M}$ has a $\mathrm{pH}$ of 7.4 without $\mathrm{pH}$ adjustment, $\mathrm{M}-1$ has a $\mathrm{pH}$ of 8.1 with $\mathrm{pH}$ adjustment) [a], and injectivity results of polymer solutions of the same polymer solution with the same shearing intensity and device, but different pH adjustment (T2 has a pH of 7.3 without pH adjustment, T2-1 has a pH of 8.0 with pH adjustment) [b]

Table 4-9: Rheological results of injected unsheared polymer solutions with different inverter surfactant concentration, referring to Figure 4-31a

\begin{tabular}{|c|c|c|c|c|c|c|c|c|c|c|}
\hline & \multirow{2}{*}{$\begin{array}{l}\text { Volume } \\
\text { specific } \\
\text { energy } \\
\text { input }\end{array}$} & \multirow{2}{*}{$\begin{array}{l}\text { Viscosity at } \\
30^{\circ} \mathrm{C} \text { from } \\
\text { Ubbelohde } \\
( \pm 5 \%)\end{array}$} & \multirow{2}{*}{$\begin{array}{l}\text { Viscosity } \\
\text { at } 150 \\
\text { rps } \\
( \pm 5 \%)\end{array}$} & \multirow{2}{*}{$\begin{array}{l}\text { Screen } \\
\text { factor } \\
( \pm 5 \%)\end{array}$} & \multirow{2}{*}{$\begin{array}{c}\mathrm{pH} \\
( \pm 5 \%)\end{array}$} & \multirow{2}{*}{$\begin{array}{c}\text { Filtration } \\
\text { ratio (FR) } \\
( \pm 5 \%)\end{array}$} & \multicolumn{2}{|c|}{$\begin{array}{c}\text { Resistance factor } \\
(R F)( \pm 0.5 \%)\end{array}$} & \multirow{2}{*}{$\begin{array}{c}\text { Injection } \\
\text { gradient (IG) } \\
\text { at } 20 P V \\
( \pm 0.5 \%)\end{array}$} & \multirow{2}{*}{$\begin{array}{c}\text { Residual } \\
\text { resistance } \\
\text { factor }(R R F) \\
( \pm 0.5 \%)\end{array}$} \\
\hline & & & & & & & $\begin{array}{l}R F \text { at } \\
3 P V\end{array}$ & $\begin{array}{l}R F \text { at } \\
20 P V\end{array}$ & & \\
\hline & {$[\mathrm{MJ} / \mathrm{m} 3]$} & [mPas] & [mPas] & {$[-]$} & {$[-]$} & {$[-]$} & {$[-]$} & {$[-]$} & {$[-]$} & {$[-]$} \\
\hline$M$ & 0 & 12.5 & 12.2 & 18.4 & 7.4 & 1.6 & 37.7 & 108.8 & 2.9 & $>>100$ \\
\hline M-1 & 0 & 12.1 & 11.8 & 19.9 & 8.1 & 1.7 & 37.4 & 90.6 & 2.4 & 86.2 \\
\hline Increase & $0 \%$ & $-3.4 \%$ & $-3.3 \%$ & $8.2 \%$ & $9.5 \%$ & - & $0.8 \%$ & $20.2 \%$ & $19.2 \%$ & >>16\% \\
\hline
\end{tabular}

Table 4-10: Rheological results of injected polymer solutions with the same shearing intensity but different inverter surfactant concentration, referring to Figure 4-31b

\begin{tabular}{|c|c|c|c|c|c|c|c|c|c|c|c|c|}
\hline & \multirow{2}{*}{$\begin{array}{l}\text { Volume } \\
\text { specific } \\
\text { energy } \\
\text { input }\end{array}$} & \multirow{2}{*}{$\begin{array}{l}\text { Viscosity at } \\
30^{\circ} \mathrm{C} \text { from } \\
\text { Ubbelohde } \\
\text { ( } \pm 5 \%)\end{array}$} & \multirow{2}{*}{$\begin{array}{c}\text { Viscosity } \\
\text { at } 150 \\
\text { rps } \\
( \pm 5 \%)\end{array}$} & \multirow{2}{*}{$\begin{array}{l}\text { Screen } \\
\text { factor } \\
( \pm 5 \%)\end{array}$} & \multirow{2}{*}{$\begin{array}{c}\mathrm{pH} \\
( \pm 5 \%)\end{array}$} & \multirow{2}{*}{$\begin{array}{c}\text { Filtration } \\
\text { ratio (FR) } \\
( \pm 5 \%)\end{array}$} & \multicolumn{3}{|c|}{$\begin{array}{l}\text { Resistance factor (RF) } \\
\text { ( } \pm 0.5 \%)\end{array}$} & \multicolumn{2}{|c|}{$\begin{array}{l}\text { Injection gradient } \\
\qquad(I G)( \pm 0.5 \%)\end{array}$} & \multirow{2}{*}{$\begin{array}{c}\text { Residual } \\
\text { resistance } \\
\text { factor } \\
(R R F) \\
( \pm 0.5 \%)\end{array}$} \\
\hline & & & & & & & $\begin{array}{l}R F \text { at } \\
3 P V\end{array}$ & $\begin{array}{l}R F \text { at } \\
30 P V\end{array}$ & $\begin{array}{l}R F \text { at } \\
60 P V\end{array}$ & $\begin{array}{l}\text { IG at } \\
30 P V\end{array}$ & $\begin{array}{l}\text { IG at } \\
60 P V\end{array}$ & \\
\hline & {$[\mathrm{MJ} / \mathrm{m} 3]$} & [mPas] & [mPas] & {$[-]$} & {$[-]$} & {$[-]$} & {$[-]$} & {$[-]$} & {$[-]$} & {$[-]$} & {$[-]$} & {$[-]$} \\
\hline $\mathrm{T} 2$ & 31.3 & 11.1 & 11.1 & 10.3 & 7.3 & 1.07 & 22.3 & 36.5 & 50.3 & 1.6 & 1.4 & 35.6 \\
\hline T2-1 & 31.3 & 10.8 & 10.8 & 10.1 & 8 & 1.1 & 18.8 & 28.5 & 39.0 & 1.5 & 1.4 & 15.8 \\
\hline Increase & $0.0 \%$ & $-2.9 \%$ & $-2.9 \%$ & $-1.6 \%$ & $9.6 \%$ & 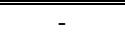 & $-15.8 \%$ & $-21.8 \%$ & $-22.5 \%$ & $\begin{array}{c}-5.0 \% \\
\end{array}$ & $-2.4 \%$ & -55.6\% \\
\hline
\end{tabular}




\section{b) Mechanical Degradation in Sand-Pack}

As the flow is not laminar through a constant diameter but shows many contractions and irregular path ways, mechanical degradation also occurs in the sand-pack. By using Equation 2-9, the mechanical degradation in the sand-pack can be determined and is expressed in percentage in Figure 4-32. Both viscosity and screen factor show less mechanical degradation, the more the polymer solution has been pre-sheared. The reduction of the mechanical degradation with the increasing pre-shearing intensity can be explained by the exponential drop of both viscosity and screen factor against the shearing intensity as shown in Figure 4-7. A significant rupturing of the chains and entanglement of the polymer chains occurs in the beginning at low shearing intensities for all shearing origins and it stays relatively constant thereafter. This means that the more the solution has been pre-sheared, the less mechanical degradation can be expected in the sand-pack, as the polymer chains already approach an asymptotic viscosity and screen factor.

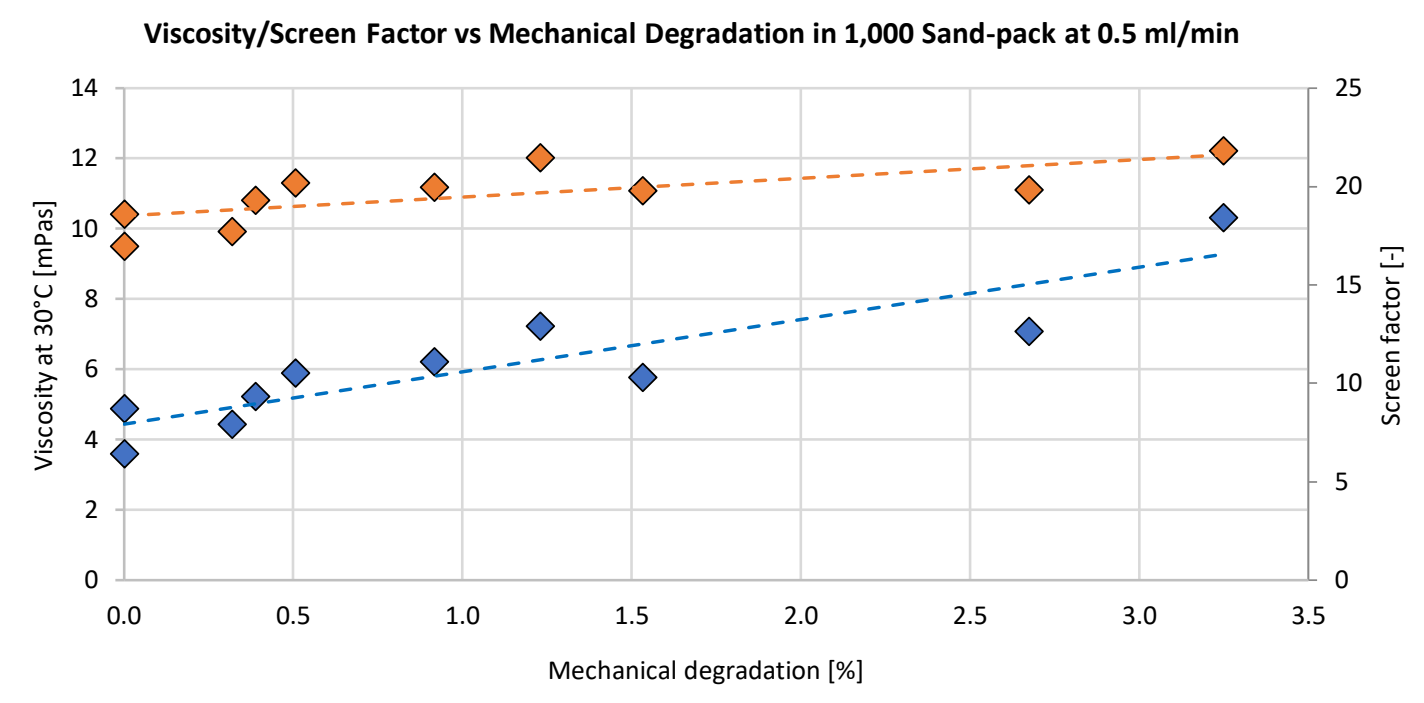

$\diamond$ Viscosity @30 $\mathrm{C}$ (measured with Ubbelohde) vs mechanical degradation

$\diamond$ Screen factor vs mechanical degradation

Figure 4-32: Viscosity measured by Ubbelohde and screen factor vs mechanical degradation in the sand-pack at $0.5 \mathrm{ml} / \mathrm{min}$ using injected solutions M, C, T1, T2, D, DT1, DT2, DT3, DT4, T3 shown in Figure 4-29 and Table 4-8

\section{c) Polymer Retention}

Polymer retention plays an important role while dealing with high-molecular-weight polymers, bearing anionic charges. Especially mechanical trapping plays a significant role and can be identified as the major source of polymer retention, when the hydrodynamic radius of the polymer is larger than the pore size. While adsorption occurs in the first pore volumes of the injected polymer solution, mechanical trapping increases with time. As the $I G$ for the tested solutions increasingly deteriorates over time or $P V$, it can be implied that mechanical trapping is a major source of polymer retention.

In Figure 4-33, the permeability reduction caused by polymer retention is shown to be dependent of the pre-shearing intensity. The permeability reduction is displayed after the injection of 3 and $30 \mathrm{PV}$ of polymer injected as well as the reduction in the residual permeability that is measured after the polymer injection with $R R F$. The permeability reduction is determined by the following equation:

$$
k_{\text {reduction }}=1-\frac{k_{w}}{k_{p}}
$$

whereby 


$$
\frac{k_{w}}{k_{p}}=\frac{R F_{\text {theo }}}{R F}
$$

As can be seen in Figure 4-33, a high permeability reduction in the sand-pack can be observed by the injected polymer solution. The reduction in permeability grows with increasing duration of polymer injection. Since polymer adsorption occurs already in very beginning of the polymer injection, this indicates that mechanical trapping is the major cause of polymer retention. Further, it can be seen that the permeability reduction declines with increasing pre-shearing intensity. This shows that by destroying the too-large molecular weight and disentangling the polymer solution, mechanical trapping can be reduced.

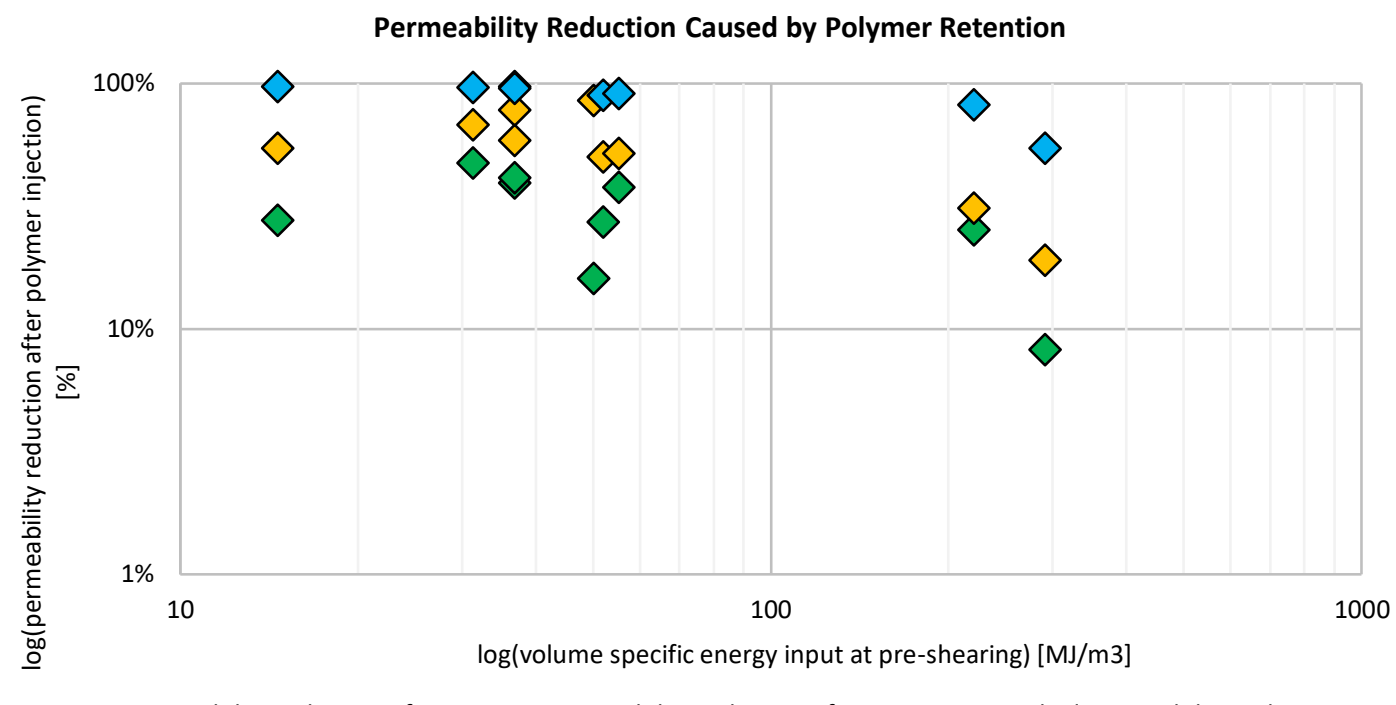

$\diamond$ Permeability reduction after 3PV $\diamond$ Permeability reduction after 30PV $\diamond$ Residual permeability reduction

Figure 4-33: \%-permeability reduction determined by the inverse of the ratio of theoretical and measured $R F$ after injecting the sheared solutions C, T1, T2, D, DT1, DT2, DT3, DT4, T3 (shown in Figure 4-29 and Table 4-8) at 3 and 30 PV as well as the residual reduction

It is difficult to precisely distinguish between the polymer retention mechanisms, such as mechanical trapping and adsorption. By determining the nitrogen in the polymer solution before and after the injection, the level of adsorption can be estimated. The Kjeldahl nitrogen is measured, which is the total nitrogen in the solution including chemical compounds such as ammonium, via ion chromatography with the Buchi B339 measuring device. The results are shown in Table 4-11, and the corresponding flooding test is shown in Figure 4-34. The polymer solution has been sheared for 30 min at $1750 \mathrm{rpm}$ with the disperser, followed by $1 \mathrm{~min}$ at 20,000 rpm.

Table 4-11: Nitrogen analysis of flooding test with a sheared polymer solution in a 1,000 mD permeable sand-pack

\begin{tabular}{|c|c|c|c|c|}
\hline & $\mathrm{pH}$ & $\begin{array}{c}\text { Viscosity at } 30^{\circ} \mathrm{C} \text { from } \\
\text { Ubbelohde ( } \pm 5 \%)\end{array}$ & $\begin{array}{c}\text { Screen factor } \\
( \pm 5 \%)\end{array}$ & $\begin{array}{c}\text { Kjeldahl - N } \\
\text { (<1mg/L) }\end{array}$ \\
\hline & {$[-]$} & [mPas] & {$[-]$} & {$[\mathrm{mg} / \mathrm{L}]$} \\
\hline Solution before polymer flooding & 7.8 & 11.3 & 10.9 & 334.2 \\
\hline Solution after polymer flooding & 7.7 & 11.1 & 10.5 & 321.9 \\
\hline Reduction & & & & -12.3 \\
\hline
\end{tabular}


Polymer Flooding in a 1,000 mD Permeable Sand-Pack with Disperser-Turrax-sheared Polymer Solution

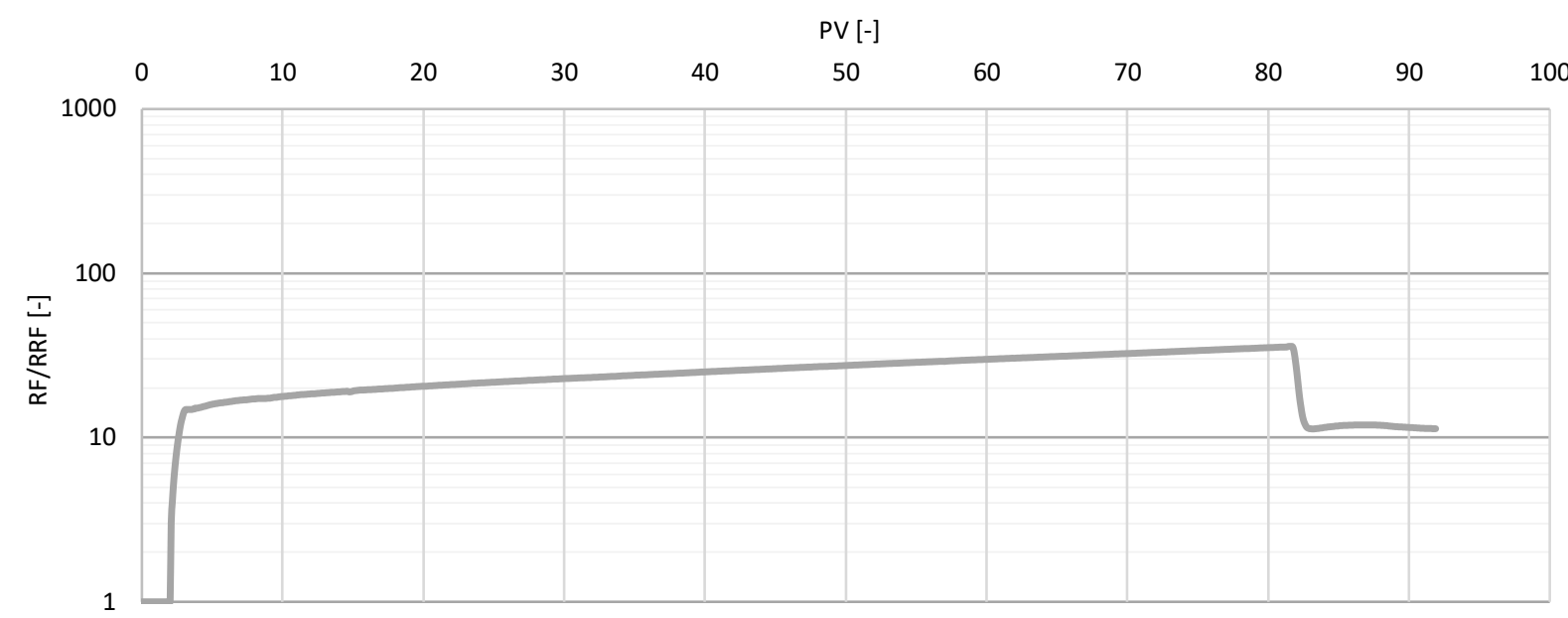

Figure 4-34: Disperser-turrax-sheared polymer injection (sheared $30 \mathrm{~min}$ at $1750 \mathrm{rpm}$ with disperser and $1 \mathrm{~min}$ at 20,000 rpm

turrax)

A nitrogen adsorption of $12.3 \pm 1$ nitrogen $\mathrm{mg} / \mathrm{L}$ polymer solution of injecting $79 \mathrm{PV}$ polymer solution into the sand cell, with a $P V$ of $6.5 \mathrm{ml}$, was determined. This gives a polymer adsorption of $0.148 \mathrm{~g}$ polymer/L polymer solution. The resulting polymer retention $\Gamma$ can then be determined as follows:

$\Gamma=\frac{V_{\text {polymer solution }}}{m_{\text {sand-pack }}} \cdot \Delta C_{\text {polymer }}=\frac{79.2 \mathrm{PV} \cdot 6.5 \mathrm{e}-3 \frac{\mathrm{L} \text { polymer solution }}{\mathrm{PV}}}{20 \mathrm{~g}} \cdot 0.148 \mathrm{e} 3 \frac{\mathrm{mg} \text { polymer }}{\text { L polymer solution }}=3.81 \frac{\mathrm{mg} \text { polymer }}{\mathrm{g} \text { sand }}$

where $V_{\text {polymer solution }}$ is the volume of polymer solution injected, $m_{\text {sand-pack }}$ is the mass of sand in the cell and $\Delta C_{\text {polymer }}$ is the difference in polymer concentration before and after injection. The resulting polymer retention can be determined to be $3.81 \mathrm{mg}$ polymer/g sand.

The accuracy of the measurement is $<1 \mathrm{mg} / \mathrm{L}$ for Kjeldahl. The total organic content (TOC) analysis could not give reproducible and reliable results, owing the nature of the emulsion polymers.

\subsubsection{Impact of Polymer and Surfactant Concentration on Pre-conditioned Polymer Solution}

Polymer and surfactant concentrations in the polymer solution play an important role regarding the rheology and injectivity behavior of the polymer solution. In the first section, the influence of a higher polymer concentration on polymer retention is investigated. Further, the impact of reduced surfactant concentration is investigated in order to observe the importance of surface tension on pre-sheared polymer solutions.

\section{a) Impact of Polymer Concentration}

In order to exclude the viscosity reduction as the source of the improved injectivity, a polymer solution with 5,000 ppm active polymer and a proportional amount of $I S-A$ of 3,000 ppm is prepared and disperser-turrax-sheared in the same manner as the compared polymer solution with 4,000 ppm active polymer and 2,400 ppm IS-A. The injectivity profile for both solutions is shown in Figure 4-35, and with additional data is given in Table 4-12. It can be seen that the $R F$-curve shows a similar behavior for both polymer concentrations, whereby the polymer solution with a higher concentration has a slightly higher $R F$, which is due to the increase in the viscosity of the 5,000 ppm polymer solution, since $R F=\frac{k_{w}}{k_{p}} \frac{\mu_{p}}{\mu_{w}}$. The $R R F$ of both injected solutions is 7.2, indicating the same polymer retention. The polymer retention, especially mechanical trapping, does not depend on the polymer concentration, but on the destruction of too-high molecular 
weight chains and chain disentanglement. Further, the surface tension of the polymer fluid is the same for both the polymer concentrations.

Polymer Flooding in a 1000mD Permeable Sand-Pack of Disperser-Turrax-sheared Polymer Solution with Different Polymer Concentrations

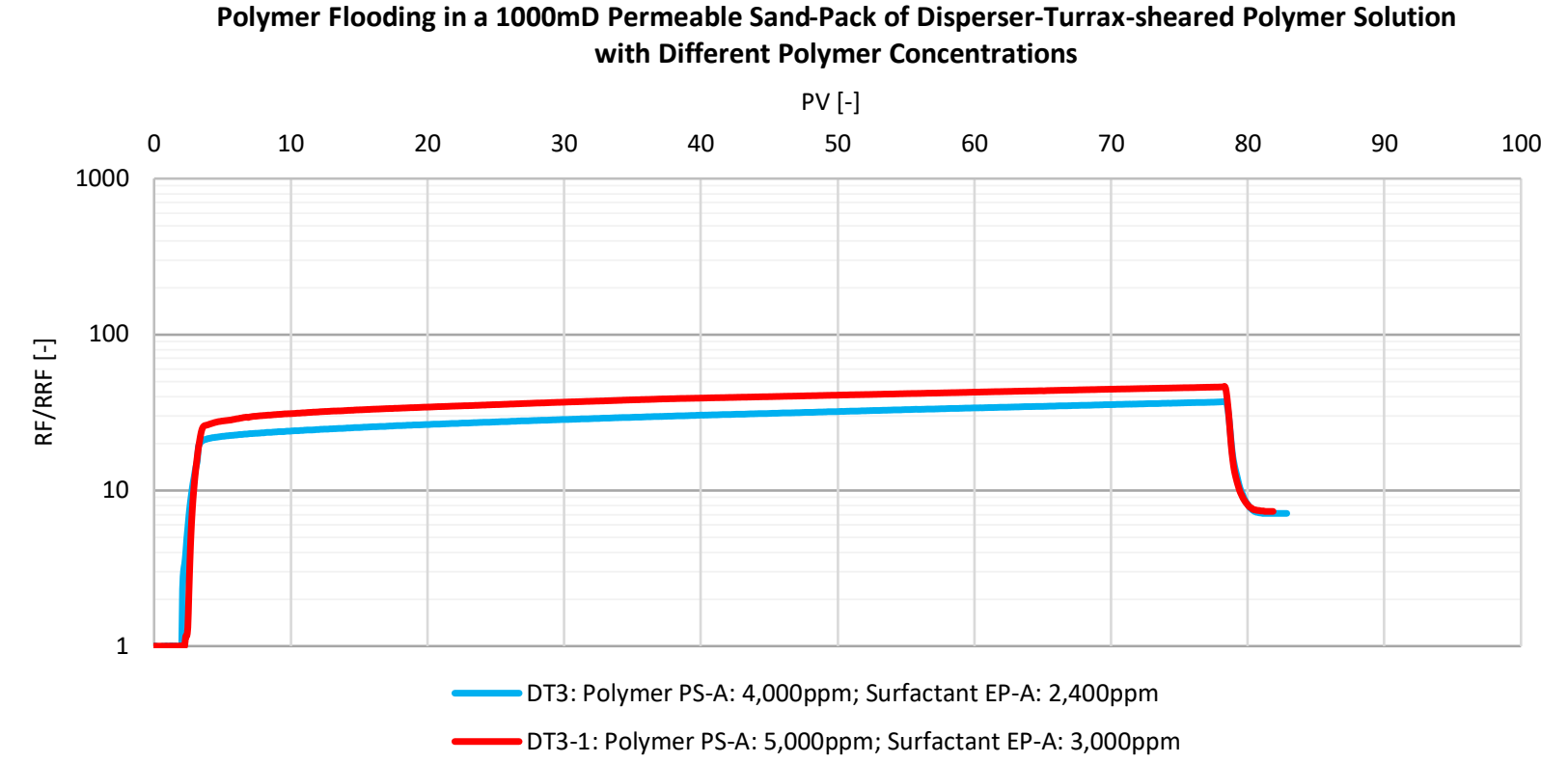

Figure 4-35: Injectivity results of polymer solutions with different polymer concentration with the same shearing intensity and devices

Table 4-12: Rheological results of injected polymer solutions with different polymer concentration with the same shearing intensity and devices, referring to Figure 4-35

\begin{tabular}{|c|c|c|c|c|c|c|c|c|}
\hline & $\begin{array}{c}\text { Polymer } \\
\text { concentration } \\
P S-A\end{array}$ & $\begin{array}{c}\text { Inverter } \\
\text { surfactant } \\
\text { concentration } \\
\text { EP-A }\end{array}$ & $\begin{array}{c}\text { Volume } \\
\text { specific } \\
\text { energy input }\end{array}$ & $\begin{array}{c}\text { Viscosity at } 30^{\circ} \mathrm{C} \\
\text { from Ubbelohde } \\
( \pm 5 \%)\end{array}$ & $\begin{array}{c}\text { Viscosity at } \\
150 \mathrm{rps} \\
( \pm 5 \%)\end{array}$ & $\begin{array}{l}\text { Screen } \\
\text { factor } \\
( \pm 5 \%)\end{array}$ & $\begin{array}{c}\text { Filtration } \\
\text { ratio (FR) } \\
( \pm 0.5 \%)\end{array}$ & $\begin{array}{l}\text { Surface Tension } \\
( \pm 0.5 \mathrm{mN} / \mathrm{m})\end{array}$ \\
\hline & [ppm] & [ppm] & {$\left[\mathrm{MJ} / \mathrm{m}^{3}\right]$} & [mPas] & [mPas] & {$[-]$} & {$[-]$} & {$[\mathrm{mN} / \mathrm{m}]$} \\
\hline DT3 & 4000 & 2400 & 55.3 & 10.5 & 11.4 & 9.3 & 1.04 & 25.3 \\
\hline Increase & $20 \%$ & $20 \%$ & $0 \%$ & $34 \%$ & $34 \%$ & $21 \%$ & - & $0 \%$ \\
\hline
\end{tabular}

\section{b) Impact of Surfactant Concentration}

The accurate choice of surfactant concentration is the key to a successful injection. First of all, the inversion of the waterin-oil polymer emulsion to a polymer solution containing oil droplets only occurs if the amount of surfactant is sufficient. Otherwise, the viscosity does not build up to its full extent. Further, the injection can only be successful if the surface tension of the polymer solution is reduced. When this happens, there is an increase in the surface of the fluid, and this enables it to flow out with better ease through the porous media. The surface tension for differently sheared polymer solutions is $25.7 \mathrm{mN} / \mathrm{m}$, implying that shearing does not change the arrangement of the surfactants.

The injected polymer solution with 1,000 ppm surfactant EP-A and 4,000 ppm polymer PS-A is shown in Figure 4-36. The solution visually appears to be inverted, as it seems to be homogeneous when injected (see Figure 4-36a). An indicator that the inversion was not fully successful is that the viscosity is half of the viscosity of the fully inverted system (see Table 4-13). Further, a part of the non-injected solution is stored for a few hours to observe the separation of polymers and emulsifiers. After a few hours of resting, the creaming is clearly visible in the stretched white clumps (see Figure 4-36b). 

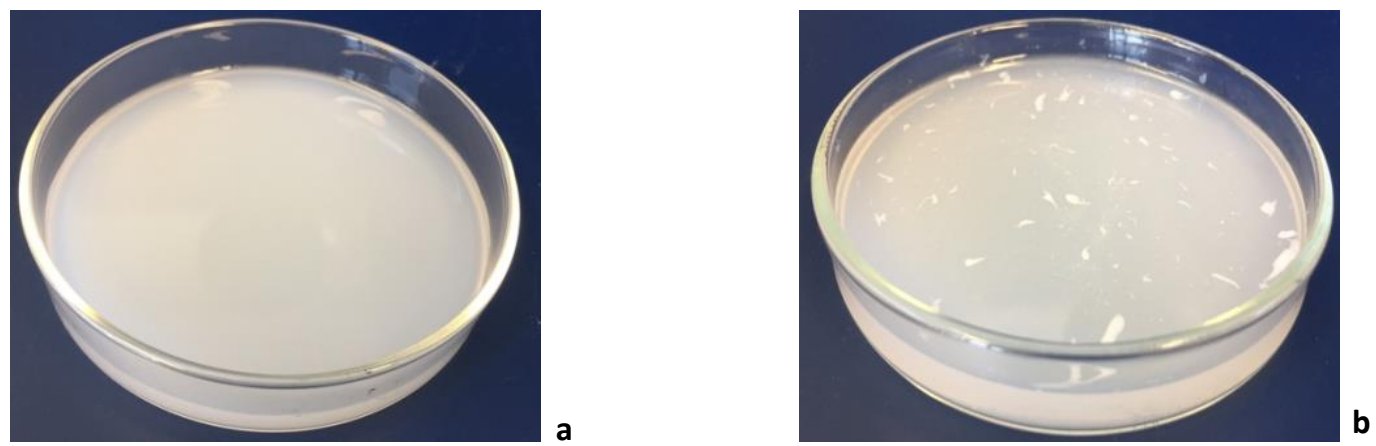

Figure 4-36: Non-inverted polymer solutions: fresh polymer solution just before injection [a], polymer solution after a few hours with precipitated polymer and emulsifiers [b]

The results of the injection of the fully inverted polymer system with 2,400 ppm surfactant (referred to as solution DT3) and of the non-inverted system with 1,000 ppm surfactant (referred to as solution DT3-2), which have both undergone the same disperser-turrax-shearing intensity, are shown in Figure 4-37a. Not only is the injectivity behavior of the noninverted solution very poor, the viscosity of the polymer solution is also not sufficient enough to achieve a feasible mobility ratio. The surface tension of both the injected solutions is measured, whereby the non-inverted polymer system solution DT3-2 has a $25 \%$ higher surface tension compared to solution DT3, meaning the fluid surface is kept low, which explains the high $R F$ and $R R F$ as compared to solution DT3.

Figure 4-37b and Table 4-14 show the injection of two polymer systems, sheared with the same shearing intensity and device. Both solutions D and D-1 have the same polymer concentration of 4,000 ppm and surfactant concentration of 2,400 ppm. While solution $D$ is immediately injected after inverting and pre-shearing the polymer solution with the disperser, solution DT-1 is separated over 13 days, in the same manner as described in section 4.3.1. Also, here, the polymer solution clears up in the middle part of the separator funnel, which indicates that there has been a reduction in the concentration of the surfactant. Since only the middle part of the polymer solution is injected after density separation, only around $40 \%$ of the oil remains in the solution. The rest of the oil is separated due to density and coalescence. As shown in Figure 4-37b, the injectivity behavior of solution D-1 is poor. Interestingly, even after the polymer injection is stopped, and the sand cell is flushed with SSW-A at $92 P V, R R F$ further increases. At $100 P V, S S W-A$ is injected together with 2,400 ppm IS-A, which immediately leads to a reduction in the $R R F$. This indicates that the separated solution contains surfactant, leaving a lower surfactant concentration in the injected solution, which increases the surface tension and leads to a poor injectivity behavior. 
Polymer flooding in a 1,000mD Permeable SandPack with Different Surfactant Concentration

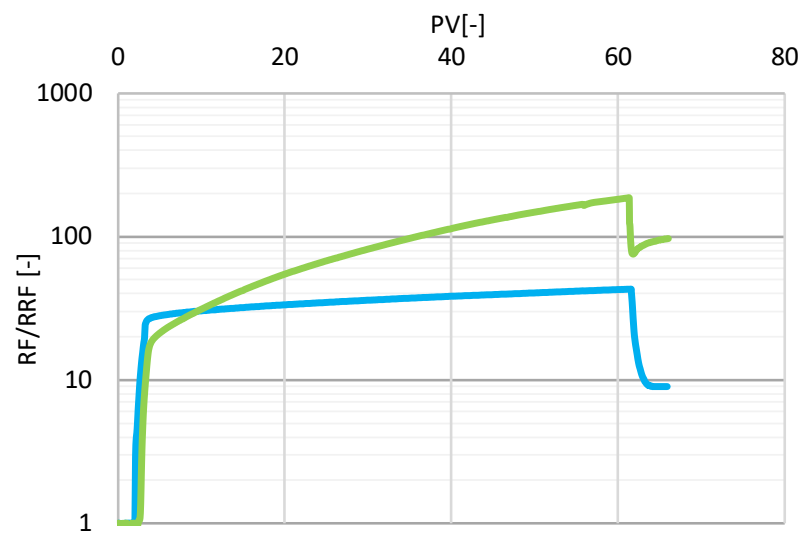

DT3: Polymer PS-A: 4,000 ppm; Inverter surfactant IS-A: 2,400 ppm

DT3-2: Polymer PS-A: 4,000 ppm; Inverter surfactant EP-A: a $1,000 \mathrm{ppm}$
Polymer Flooding in a 1,000mD Permeable SandPack with Different Surfactant Concentration

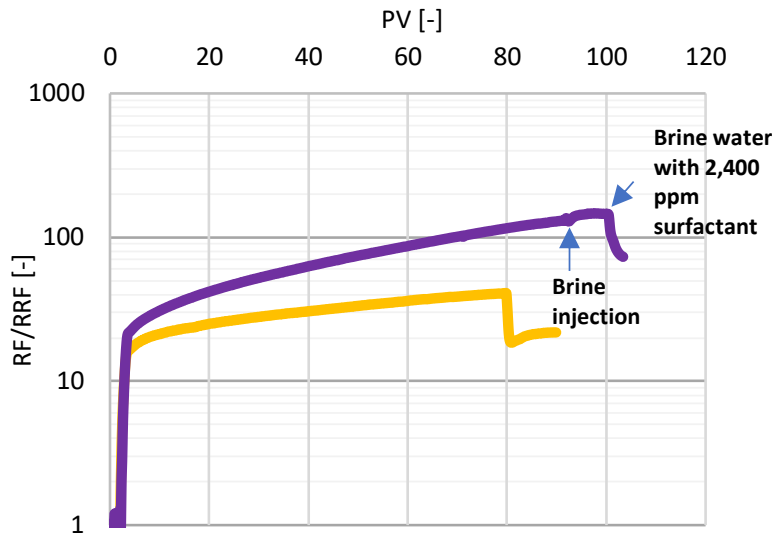

D: Fresh polymer solution

D-1: 12 days old polymer solution (separated in funnel by $\mathbf{b}$ density and coalesence)

Figure 4-37: Injectivity results of polymer solutions sheared the same way, with the same polymer concentration of 4,000 ppm and different surfactant concentration [a], and injectivity results of polymer solutions, sheared the same way with different surfactant concentration. $D$ is the fresh polymer solution $P S-A$, and D-1 is PS-A separated over 13 days with a separator funnel [b]

Table 4-13: Rheological results of injected polymer solutions with the same shearing intensity but different inverter surfactant concentration, referring to Figure 4-37a

\begin{tabular}{|c|c|c|c|c|c|c|c|c|}
\hline & $\begin{array}{c}\text { Polymer } \\
\text { concentration } \\
\text { PS-A }\end{array}$ & $\begin{array}{c}\text { Inverter } \\
\text { surfactant } \\
\text { concentration } \\
E P-A\end{array}$ & $\begin{array}{c}\text { Volume } \\
\text { specific } \\
\text { energy } \\
\text { input }\end{array}$ & $\begin{array}{l}\text { Viscosity at } 30^{\circ} \mathrm{C} \\
\text { from Ubbelohde } \\
( \pm 5 \%)\end{array}$ & $\begin{array}{l}\text { Viscosity at } \\
150 \text { rps } \\
( \pm 5 \%)\end{array}$ & $\begin{array}{l}\text { Screen } \\
\text { factor } \\
( \pm 5 \%)\end{array}$ & $\begin{array}{c}\text { Filtration } \\
\text { ratio }(F R) \\
( \pm 5 \%)\end{array}$ & $\begin{array}{c}\text { Surface } \\
\text { tension } \\
( \pm 0.5 \mathrm{mN} / \mathrm{m})\end{array}$ \\
\hline & [ppm] & [ppm] & {$\left[\mathrm{MJ} / \mathrm{m}^{3}\right]$} & [mPas] & [mPas] & {$[-]$} & {$[-]$} & {$[\mathrm{mN} / \mathrm{m}]$} \\
\hline DT3 & 4000 & 2400 & 55.3 & 10.5 & 11.4 & 9.3 & 1.04 & 25.4 \\
\hline DT3-2 & 4000 & 1000 & 55.3 & 7.0 & 7.8 & 8.8 & 1.03 & 33.7 \\
\hline Increase & $0 \%$ & $-140 \%$ & $0 \%$ & $-51 \%$ & $-47 \%$ & $-6 \%$ & - & $+25 \%$ \\
\hline
\end{tabular}

Table 4-14: Rheological results of injected polymer solutions with the same shearing intensity but different inverter surfactant concentration, referring to Figure 4-37b

\begin{tabular}{|c|c|c|c|c|c|c|c|c|}
\hline & Description & $\begin{array}{c}\text { Polymer } \\
\text { concentration } \\
\text { PS-A }\end{array}$ & $\begin{array}{c}\text { Inverter } \\
\text { surfactant } \\
\text { concentration } \\
E P \text { - } A \\
\end{array}$ & $\begin{array}{l}\text { Volume } \\
\text { specific } \\
\text { energy } \\
\text { input } \\
\end{array}$ & $\begin{array}{l}\text { Viscosity at } \\
30^{\circ} \mathrm{C} \text { from } \\
\text { Ubbelohde } \\
( \pm 5 \%) \\
\end{array}$ & $\begin{array}{c}\text { Viscosity at } \\
150 \text { rps } \\
( \pm 5 \%)\end{array}$ & $\begin{array}{l}\text { Screen } \\
\text { factor } \\
( \pm 5 \%)\end{array}$ & $\begin{array}{c}\text { Filtration } \\
\text { ratio (FR) } \\
( \pm 5 \%)\end{array}$ \\
\hline & & [ppm] & [ppm] & {$\left[\mathrm{MJ} / \mathrm{m}^{3}\right]$} & [mPas] & [mPas] & {$[-]$} & {$[-]$} \\
\hline D & Fresh & 4000 & 2400 & 14.6 & 12.0 & 12.0 & 12.9 & 1.08 \\
\hline D-1 & $\begin{array}{c}\text { In separator funnel } \\
\text { for } 12 \text { days, } \\
\text { injection only of } \\
\text { middle part } \\
\end{array}$ & 4000 & 2400 & 14.6 & 11.40 & 11.50 & 13.2 & 1.7 \\
\hline Increase & - & $0 \%$ & $0 \%$ & $0 \%$ & $-5 \%$ & $-4 \%$ & $2 \%$ & - \\
\hline
\end{tabular}




\section{Conclusion and Recommendation}

The study investigated on the injectivity characteristics of an acrylamide-based copolymer emulsion system for EOR application by using synthetic sea water SSW-A as make-up water. The studied high-molecular-weight acrylamide-based copolymer inverse emulsion system PS-A has a polymer concentration of 4,000 ppm and an inverter surfactant concentration IS-A of 2,400 ppm. The continuous water phase uses a synthetic sea water SSW-A having 50,000 ppm TDS, whereby $2,600 \mathrm{ppm}$ are divalent cations, interacting with the polymer chains.

The following conclusions could be drawn:

- Rheological master curves have been determined for the given polymer solution, exposed to different shearing mechanisms exerted by a disperser, Ultra-Turrax and pressure-driven flow into a capillary. A strong trend occurs between the screen factor and viscosity by pre-conditioning the polymer solution with different devices, whereby the screen factor measures the transition of the polymer from coiled to stretched state, and the viscometer measures the viscosity of the entire solution. Further, another master curve of mechanical degradation events has been established for the given system by determining the volume specific energy input that is imposed by the shearing device to the polymer solution with renowned empirical turbine power correlations. The curves show a strong trend, whereby both the viscosity and the screen factor approach an asymptotic value with the increase in energy input.

- The sand-packed flooding experiments revealed a poor injectivity behavior of the unsheared polymer solution, which is caused significantly by mechanical trapping. The viscosity of the unsheared polymer solution is $12.5 \mathrm{mPas}$, the screen factor is 18.4 and the $\mathrm{pH}$ is 7.4. The filtration ratio of 1.6 already indicates a poor injectivity behavior. The injection in a 1,000 mD sand cell gives an injection gradient of 3.7 over $30 \mathrm{PV}$. Also, a change in the concentration of the inverter surfactant did not influence the injectivity performance.

- Conditioning the polymer solution by pre-shearing the polymer solution highly improves the injectivity behavior of the polymer solution. By pre-shearing the polymer solution with a disperser and imposing $15 \mathrm{MJ} / \mathrm{m}^{3}$ energy to the polymer solution, the injectivity gradient improves from 3.7 to 1.6 by $230 \%$ over $30 \mathrm{PV}$, while the viscosity is reduced by $1.6 \%$ and the corresponding screen factor by $30 \%$ from 18.4 to 12.9 . The $\mathrm{pH}$ is increased to 7.8 . This shows a high amount of disentanglement occurs and only a small amount of chains rupture. The polymer solutions sheared with a turrax also show a significantly improved injectivity behavior. However, a comparably higher degradation of viscosity is necessary for gaining a favorable injectivity. To reach a comparable injection to the mentioned dispersersheared solution, the turrax imposes $31 \mathrm{MJ} / \mathrm{m} 3$ and reduces the viscosity to $11.1 \mathrm{mPas}$ or by $11.2 \%$ and the screen factor is reduced 10.3 or by $44.0 \%$. The $\mathrm{pH}$ of the turrax-sheared polymer solution is 7.3. By increasing the $\mathrm{pH}$ of the turrax-sheared polymer solution the injectivity can be improved with a higher $\mathrm{pH}$, since the $\mathrm{pH}$ influences the charge of the solid surface. Thus, the difference in injectivity of disperser-sheared and turrax-sheared polymer solutions can be found in a difference in the $\mathrm{pH}$. The sheared polymer solutions through a contraction into a $1 \mathrm{~mm}$-capillary at 20 bar shows a poor injectivity behavior by imposing $50 \mathrm{MJ} / \mathrm{m}^{3}$. The injectivity gradient is worsened to 5.7 , even though the filtration ratio was found to be 1.05, which shows that the filtration ratio is only an indication for a favorable injectivity performance. Still, flooding tests are inevitable. Both viscosity and screen factor are reduced to $10.1 \mathrm{mPas}$ or by $19.3 \%$, and to 8.7 or by $52.7 \%$ respectively. This indicates that there is a different resulting polymer structure than when sheared with agitators.

In future work, flooding tests with reservoir cores should be performed with differently sheared polymer solutions in order to validate the injectivity behavior through solid rock and have further understanding of the polymer solution at 
reservoir conditions. Further, conditions such as reservoir pressure and temperature should be considered since it highly influences the performance of the polymer solution. Also, the MWD should be determined using appropriate GPC columns to detect the changes especially at the high-end of the MWD caused by pre-shearing. This also allows to determine the differences in pre-shearing with various shearing devices. Master curves can further be investigated using different polymer and surfactant types and concentrations in order to validate whether this behavior exists for all degraded polymer solutions.

In conclusion, this study showed that specific pre-shearing of inverse polymer emulsion system improves the injectivity behavior significantly. This finding can improve the utilization of polymer flooding significantly and reduce the phenomena of mechanical trapping. Further rheological master curves for the polymer systems have been established and proven to be independent of shear-origin, whereas the injectivity behavior depends on the source of shearing. 


\section{Nomenclature}

Mark-Houwink constant

C Polymer concentration

$C^{C} \quad$ Critical polymer concentration, transitions of semi-dilute to concentrated regime

$C^{*} \quad$ Transition of dilute to semi-dilute regime

$d_{i} \quad$ Diameter of impeller

EP-A Polymer emulsion at stake

$E_{v} \quad$ Volume specific energy input

FR Filtration ratio

$G^{*} \quad$ Complex shear modulus

$G^{\prime} \quad$ Loss modulus

$G^{\prime \prime} \quad$ Storage modulus

$H L B \quad H y d r o p h i l i c-l i p o p h i l i c$ balance

IG Injectivity gradient after a specific amount of pore volumes injected

IS-A Inverter surfactant at stake

$k \quad$ Permeability

$\mathrm{K} \quad$ Consistency factor in power law model

$K_{M H} \quad$ Mark Houwink constant

$k_{\text {reduction }}$ Permeability reduction

$K_{U b b} \quad$ Ubbelohde constant

$m \quad$ Mass

$M_{W} \quad$ Molecular weight

MWD Molecular weight distribution

$n \quad$ Flow factor for power law model

$N_{p} \quad$ Power number/ Newton number

$n_{r} \quad$ Rotational speed

OPEX Operating expense

$P \quad$ Power input

PS-A Polymer solution at stake including polymer emulsion, inverter surfactant and synthetic sea water

PV Pore volume

$r \quad$ Radius of capillary

$R \quad$ Average radius of flow path in porous media

$R_{e} \quad$ Reynolds number for agitators

$R F \quad$ Resistance factor

$R R F \quad$ Residual resistance factor

SF Screen factor

SSW-A Synthetic sea water/brine water at stake

$\mathrm{t} \quad$ Measuring time

TDS Total dissolved solids

$u_{D} \quad$ Darcy or superficial velocity

$u_{p} \quad$ Darcy velocity of polymer solution

$u_{s} \quad$ Darcy velocity of solvent

$V \quad$ Volume of solution

$\dot{\gamma} \quad$ Shear rate

$\dot{\gamma}_{c 1} \quad$ First critical shear rate

$\dot{\gamma}_{c 2} \quad$ Second critical shear rate

$\Gamma \quad$ Polymer retention

$\Delta L \quad$ Length gradient 


$\begin{array}{ll}\Delta P & \text { Pressure gradient } \\ \Delta P_{p} & \text { Pressure gradient of polymer flooding } \\ \Delta P_{w} & \text { Pressure gradient of (brine) water flooding } \\ \eta & \text { Apparent viscosity } \\ \eta_{\text {eff }} & \text { Effective apparent viscosity } \\ \lambda & \text { Mobility ratio } \\ \lambda_{p} & \text { Mobility ratio of polymer } \\ \lambda_{w} & \text { Mobility ratio of (brine) water } \\ \mu & \text { Dynamic viscosity } \\ \mu_{i n i t} & \text { Viscosity before mechanical degradation } \\ \mu_{m} & \text { Viscosity after mechanical degradation } \\ \mu_{p} & \text { Polymer viscosity } \\ \mu_{s} & \text { Solvent viscosity } \\ \mu_{w} & \text { Water viscosity } \\ v & \text { Kinematic viscosity } \\ v_{i} & \text { Interstitial velocity } \\ \bar{v}_{i} & \text { Average interstitial velocity } \\ \rho & \text { Fluid density } \\ \tau & \text { Shear stress } \\ \phi & \text { Porosity }\end{array}$




\section{References}

Al Hashmi, A. R., Al Maamari, R. S., Al Shabibi, I. S., Mansoor, A. M., Zaitoun, A., \& Al Sharji, H. H. (2013). Rheology and mechanical degradation of high-molecular-weight partially hydrolyzed polyacrylamide during flow through capillaries. Journal of Petroleum Science and Engineering 105, 100-106.

Bates, R., Fondy, P., \& Corpstein, R. (1963). An Examination of Some Geometric Parameters of Impeller Power. In H. Hulburt, Industrial and Engineering Chemistry Process Design and Development (Vol. 2, pp. 310-314). Dayton, Ohio, USA: ACS Publications.

Brookfield. (n.d.). Operating Instructions Manual No. M08-219-B1211. Middleboro, USA: Brookfield Engineering Labs., Inc.

Chauveteau, G. (1986). Fundamental Criteria in Polymer Flow Through Porous Media And Their Importance in the Performance Differences of Mobility-Control Buffers. In Advances in Chemistry (Vol. 213, pp. 227-267). RueilMalmaison, France: American Chemical Society.

Clasen, C., \& Kulicke, W. M. (2004). Viscosimetry of Polymers and Polyelectrolytes. Hamburg, Germany: Springer-Verlag Berlin Heidelberg.

Dake, L. (2008). Fundamentals of Reservoir Engineering. Amsterdam, Netherlands: Elsevier.

Delshad, M., Pope, G., \& Sepehrnoori, K. (2008). Mechanistic Interpretation and SPE-113620-MS: Utilization of Viscoelastic Behavior of Polymer Solutions for Improved Polymer-Flood Efficiency. SPE Symposium on Improved Oil Recovery, 20-23 April, Tulsa, Oklahoma, USA. Society of Petroleum Engineers.

Duffy, J. (2015). Measuring the rheology of polymer solutions. Worcestershire, UK: Malvern Instruments Limited.

Goodyer, S. (2015). Measuring Polymers using a Rotational Rheometer in Oscillatory Mode. Graz: Anton Paar Ltd.

Graillat, C., Pichot, C., Guyot, A., \& El Aasser, M. (1986). Inverse Emulsion Polymerization of Acrylamide. I. Contribution To The Study of Some Mechanistic Aspects. Journal of Polymer Science: Part A: Polymer Chemistry, Vol. 24, 427449.

Hershey, H. C., \& Brodkey, R. (1988). Transport Phenomena: A Unified Approach (Vol. 1). Columbus, Ohio, USA: Brodkey Publishing.

Hirasaki, G., \& Pope, G. (1974, August). SPE-4026-PA: Analysis of Factors Influencing Mobility and Adsorption in the Flow of Polymer Solution Through Porous Media. Society of Petroleum Engineers Journal, 14, 337-346.

IKA. (n.d.). Ultra-Turrax-Manual: Dispergierwerkzeuge/ Dispersion tools/ Outils dispersants.

Jennings, R., Rogers, J., \& West, T. (1971, March). SPE-2867-PA: Factors Influencing Mobility Control By Polymer Solutions. Journal of Petroleum Technology, 391-401.

Jouenne, S., Chakibi, H., \& Levitt, D. (2017). SPE-186103-PA: Polymer Stability After Successive Mechanical-Degradation Events. SPE Journal 2017.

Jouenne, S., Klimenko, A., \& Levitt, D. (2016). SPE-179631-MS: Tradeoffs between Emulsions and Powder Polymers for EOR. Tulsa, USA: Society of Petroleum Engineers. 
Lake, L. W. (1989). Fundamentals of Enhanced Oil Recovery. Upper Saddle River, New Jersey, USA: Prentice Hall.

Maerker, J. (1975). SPE-5101-PA: Shear Degradation of Partially Hydrolyzed Polyacrylamide Solutions. Society of Petroleum Engineers Journal, 311-322.

Masuda, Y., Tang, K., Miyazawa, M., \& Tanaka, S. (1992). SPE-19499-PA: 1D Simulation of Polymer Flooding Including the Viscoelastic Effect of Polymer Solution. In SPE Reservoir Engineering (Vol. 7, pp. 247-252). Society of Petroleum Engineers.

Reining, R. (2011). Visco handbook - Theory and Application of Visometry with Glass Capillary Viscometers. Rye Brook / N.Y., USA: SI Analytics GmbH.

Rushton, J. H., Costrich, E., \& Everett, H. J. (1950). Power characteristics of mixing impellers. In Chemical Engineering Progress 46 (pp. 395-479). American Institute of Chemical Engineers.

Sanchez Perez, J., Rodriguez Procel, E., \& Casas Lopez, J. (2006). Shear rate in stirred tank and bubble column bioreactors. Chemical Engineering Journal $124,1-5$.

Saurabh, M., Achinta, B., \& Mandal, A. (2013). Effect of Polymer Adsorption on Permeability Reduction in Enhanced Oil Recovery. Journal of Petroleum Engineering.

Seright, R. (1983). SPE-9297-PA: The Effects of Mechanical Degradation and Viscoelastic Behavior on Injectivitiy of Polyacrylamide Solutions. SPE Journal 1983, 415-485.

Sheng, J. (2010). Modern Chemical Enhanced Oil Recovery (Vol. 1). Lubbock, USA: Gulf Professional Publishing.

Sorbie, K. S. (1991). Polymer-Improved Oil Recovery. Edinburgh, Scottland: Springer Science+Business Media New York.

Szabo, M. T. (1975). SPE-4668-PA: Some Aspects of Polymer Retention in Porous Media Using a C14-Tagged Hydrolyzed Polyacrylamide. Society of Petroleum Engineers Journal, 323-337.

Wang, Y., Li, B. H., Xiong, X., Wang, B., \& Zhang, J. (2010). Universal scaling description of the strain-softening behavior in the semidilute uncross-linked polyacrylamide-water solution. The Royal Society of Chemistry, 3318-3324.

Zaitoun, A., Makakou, P., Blin, N., Al-Maamari, R., Al-Hashmi, A., Abdel-Goad, M., \& Al-Sharji, H. (2011). SPE-141113-MS: Shear Stability of EOR Polymers. The Woodlands, USA: Society of Petroleum Engineers. 